\title{
Spin-Dependent Phenomena in Semiconductor Micro-and Nanoparticles-From Fundamentals to Applications
}

\author{
Vladimir M. Fomin $1,2,3, *$ (D) and Victor Yu. Timoshenko ${ }^{2,4,5}$ (D) \\ 1 Laboratory of Physics and Engineering of Nanomaterials, Department of Theoretical Physics, \\ Moldova State University, strada A. Mateevici 60, MD-2009 Chisinau, Moldova \\ 2 National Research Nuclear University “MEPhI”, Kashirskoe shosse 31, 115409 Moscow, Russia; \\ timoshen@physics.msu.ru \\ 3 Institute for Integrative Nanosciences, Leibniz IFW Dresden, Helmholtzstraße 20, \\ D-01069 Dresden, Germany \\ 4 Lomonosov Moscow State University, Faculty of Physics, Leninskie Gory 1, 119991 Moscow, Russia \\ 5 Lebedev Physical Institute of the Russian Academy of Sciences, 53 Leninskiy Prospekt, \\ 119991 Moscow, Russia \\ * Correspondence: v.fomin@ifw-dresden.de; Tel.: +49-351-4659-780; Fax: +49-351-4659-782
}

Received: 18 June 2020; Accepted: 15 July 2020; Published: 20 July 2020

check for updates

\begin{abstract}
The present overview of spin-dependent phenomena in nonmagnetic semiconductor microparticles (MPs) and nanoparticles (NPs) with interacting nuclear and electron spins is aimed at covering a gap between the basic properties of spin behavior in solid-state systems and a tremendous growth of the experimental results on biomedical applications of those particles. The first part of the review represents modern achievements of spin-dependent phenomena in the bulk semiconductors from the theory of optical spin orientation under indirect optical injection of carriers and spins in the bulk crystalline silicon (c-Si) - via numerous insightful findings in the realm of characterization and control through the spin polarization - to the design and verification of nuclear spin hyperpolarization in semiconductor MPs and NPs for magnetic resonance imaging (MRI) diagnostics. The second part of the review is focused on the electron spin-dependent phenomena in Si-based nanostructures, including the photosensitized generation of singlet oxygen in porous $\mathrm{Si}$ and design of Si NPs with unpaired electron spins as prospective contrast agents in MRI. The experimental results are analyzed by considering both the quantum mechanical approach and several phenomenological models for the spin behavior in semiconductor/molecular systems. Advancements and perspectives of the biomedical applications of spin-dependent properties of Si NPs for diagnostics and therapy of cancer are discussed.
\end{abstract}

Keywords: Si micro-and nanoparticles; optically induced dynamic nuclear polarization; optical spin orientation; nuclear spin hyperpolarization; photosensitized generation of singlet oxygen; magnetic resonance imaging; diagnostics and therapy of cancer

\section{Introduction}

Semiconductor microparticles (MPs) and nanoparticles (NPs) exhibit interesting electronic, optical and magnetic properties, which depend on a preferential orientation of electron and nuclear spins in those particles. These properties are essential for their biomedical applications. Spatial confinement of charge carriers (electron and holes) in a semiconductor nanostructure results in an increase of the spin-lattice relaxation time. Going from itinerant to immobile, fully-localized electrons, while inducing the hyperfine dephasing, can be also beneficial in quenching the spin-lattice relaxation. The dynamic 
nuclear polarization in semiconductor nano- and microstructures opens fascinating prospects for creation of new efficient contrast agents in magnetic resonance imaging (MRI), which is a powerful diagnostic tool in biomedicine. Perspective applications of silicon (Si) MPs and NPs in hyperpolarized ${ }^{29} \mathrm{Si}$ MRI are discussed in Reference [1]

Si nanocrystals (nc-Si) in electrochemically prepared porous $\mathrm{Si}$ are found to be a sensitizer of the photoinduced generation of singlet oxygen, which is a highly reactive form of molecular oxygen. The photosensitized singlet oxygen generation by nc-Si with adsorbed molecular oxygen is explained by spin-dependent energy transfer from excitons confined in nc-Si to molecular oxygen in the ground triplet state. This type of oxygen generation is promising for application of nc-Si based NPs and MPs in photodynamic therapy (PDT) of cancer [2]. Both the bioimaging and therapeutic functionality of Si NPs can be combined in an approach called as theranostics (therapy + diagnostics) of cancer, which is recently an urgent task of modern nanomedicine [3].

Importantly, nanostructuring of semiconductor materials provides an avenue for a significant control over the dynamic characteristics [4,5], including those which determine the spin relaxation [6]. For instance, spin-lattice relaxation times derived in germanium quantum wells (Ge QWs) (Figure 1) approach $5 \mu$ s and are substantially longer than the one reported for conduction electrons in bulk Ge at the same temperatures $(0.02$ to $0.9 \mu \mathrm{s})$. By spatially separating conduction band electrons residing in the Ge QW from their parent donor atoms embedded in the SiGe barriers, the impurity-induced bottleneck pertaining to experiments utilizing bulk Ge wafers was prevented and long-lived spins were resolved despite the low temperature operation.

a

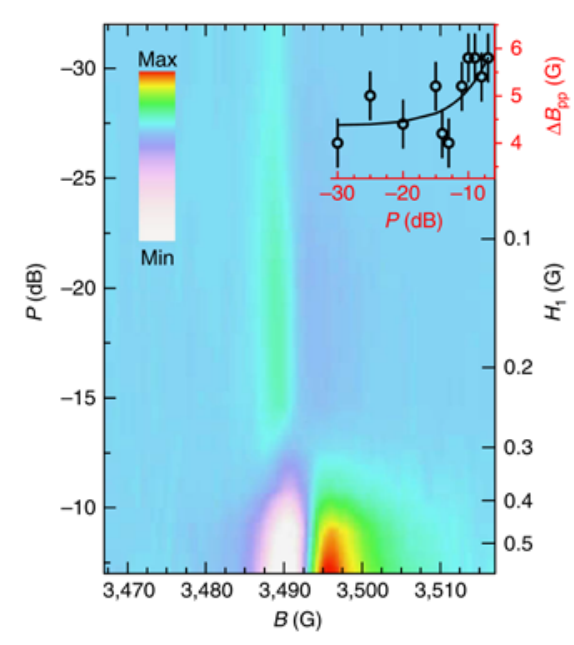

b

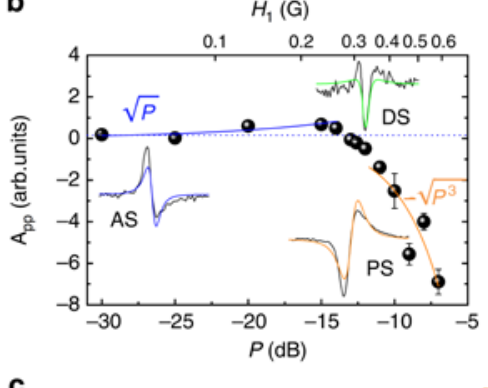

c

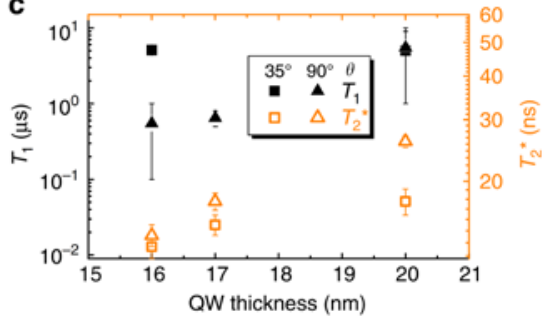

Figure 1. (a) Color-coded intensity map of the conduction electron spin resonance peak in $17 \mathrm{~nm}$ Ge quantum wells $(\mathrm{QWs})$ as a function of the microwave power $(P)$. (b) Peak-to-peak amplitude $\left(A_{\mathrm{pp}}\right)$ of the peaks shown in (a) versus $P$. (c) Values of spin-lattice relaxation time $T_{1}$ and ensemble dephasing time $T_{2}^{*}$ obtained from electron spin resonance peaks in QWs with different thickness. (Reprinted from Reference [6], ( ( ) (2016) Springer Nature. This article is licensed under a Creative Commons Attribution 4.0 International License http://creativecommons.org/licenses/by/4.0/).

As emphasized in Reference [7], the relative strength of modulation of the hyperfine coupling with nuclei by lattice vibrations and the spin-orbit interaction are different for various materials. In $\mathrm{Si}$, where the spin-orbit interaction is much weaker than in GaAs, the dominant spin-flip mechanism in the case of the phonon-assisted transitions between the Zeeman levels of the usual impurity was found to be the modulation of the hyperfine coupling with nuclei by lattice vibrations. The magnitude of the calculated spin-phonon relaxation rates in parabolic lens-shaped III-V quantum dots due to the phonon modulation of the spin-orbit interaction in presence of an external magnetic field through the deformation-potential 
and piezoelectric electron-phonon couplings increases when the dot size is increased and in the region $R>10 \mathrm{~nm}$ depends weakly on the size [8]. Correspondingly, the spin-phonon relaxation time increases when the dot size is decreased in the region below $10 \mathrm{~nm}$. A few further examples of the role of confinement in Si NPs on the spin-lattice relaxation time are provided in Section 2.

\section{Dynamic Nuclear Polarization in Semiconductor Nano-and Microstructures}

The most common ways to polarize nuclear spins are optical pumping, chemical reaction or direct transfer of spin angular momentum from electron to nuclear spins called dynamic nuclear polarization (DNP) [9]. There exists a significant difference in thermal polarization between nuclear spins and electron spins. At cryogenic temperatures, while typically less than $1 \%$ of nuclear spins are polarized under static magnetic fields of around $10 \mathrm{~T}$, the polarization of electron spins is typically larger than $90 \%$ [10]. Thus, DNP is caused by aligning the nuclear spins induced by the alignment of the electron spins [1].

In Section 2, an overview is given to DNP in semiconductor nano- and microstructures governed by optical pumping (Section 2.1.) along with the microwave irradiation (Section 2.2.). Because of significantly weaker absorption, the latter allows one to achieve much thicker spatial regions of nuclear polarization than the former. DNP by microwave irradiation permits design of the optimal materials (MPs, NPs) and properties (sizes, surface properties) to enhance nuclear polarization for MRI applications [11].

The presence of imperfections in the crystalline structure, for example, impurities and defects, such as dangling bonds (DBs), in a semiconductor opens up efficient additional—to the coupling with the electrons-channels for relaxation of the nuclear spins (see Section IX C in Reference [12]) polarized due to the optical pumping, what makes DNP by this mechanism less robust with respect to the purity of materials selection as compared to the case of the microwave irradiation.

\subsection{Optically Induced DNP in Semiconductors}

\subsubsection{First Demonstration of DNP}

Vividly developing applications of spintronics (see for example Reference [13]) Ref, in particular, in biomedicine, require a secure control over the spin polarization in semiconductors. The first demonstration of an enhanced DNP was obtained by optical pumping in very pure $n$-type $\mathrm{Si}$ (with the concentration $5 \times 10^{16} \mathrm{~m}^{-3}$ of phosphorus atoms, which was much lower than the concentration $2 \times 10^{17} \mathrm{~m}^{-3}$ of conduction electrons) [14,15] (see Figure 2). Optically induced DNP in semiconductors can be achieved in two ways-(i) saturation of the electronic spin magnetization with an unpolarized light, which generates an equal number of spins up and spins down and (ii) production of highly polarized conduction electrons by irradiation with circularly polarized light, which generates spin-polarized electrons and holes [14].

\subsubsection{Principle of DNP}

The process of DNP occurred by off-center irradiation of the resonance of paramagnetic centers, which were present at low concentration in the sample [9]. Its importance was shown to far exceed that of the other methods of nuclear polarization.

Spin-temperature theory was initially suggested for the investigation of nuclear magnetism in solids and then evolved into an important tool for the explanation of NMR in solids. A first extension of the theory to extremely low temperatures, for systems of nuclear spins at normal concentrations, led to the understanding of nuclear magnetic cooperative ordering. A second extension of the spin-temperature theory, to dilute electronic spin systems both at high and low temperatures, led to the unveiling of several physical mechanisms contributing to dynamic polarization. 


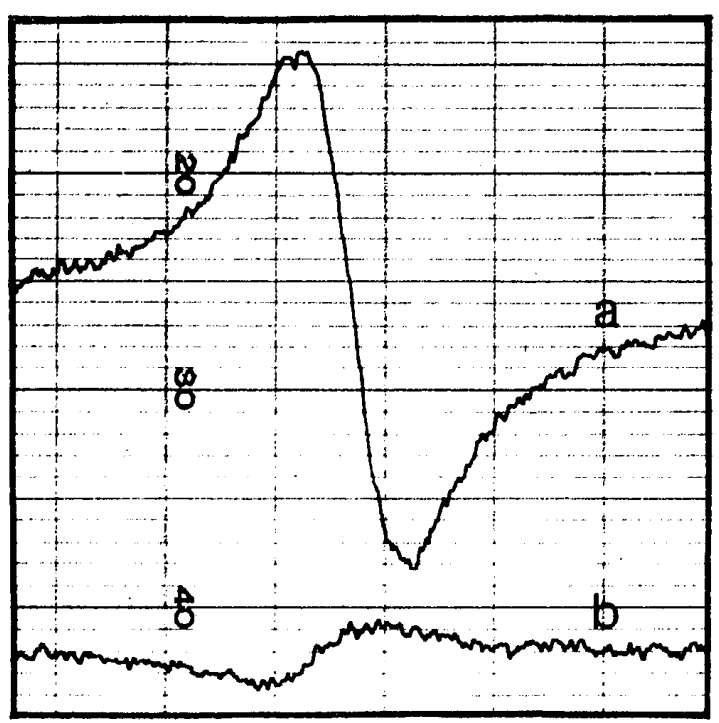

Figure 2. (a) Signal proportional to the derivative of the ${ }^{29} \mathrm{Si}$ nuclear magnetization obtained in a dc magnetic field $1 \mathrm{G}$ after $21 \mathrm{~h}$ of irradiation with circularly polarized light at $77 \mathrm{~K}$. (b) Signal proportional to the derivative of the equilibrium Si nuclear magnetization in a dc magnetic field $6 \mathrm{kG}$ at $300 \mathrm{~K}$. (Reprinted figure with permission from Reference [14] (C) (1968) by the American Physical Society).

The basic hypothesis in the spin-temperature theory is that the evolution of an isolated system of a big number of interacting spins leads to an equilibrium state characterized by a temperature. In fact, spin systems are never completely isolated. Therefore, the concept of spin temperature is meaningful only if the rate of achievement of internal equilibrium in the spin system is much faster than the spin-lattice relaxation rate. Variation of polarization and of dipolar inverse temperature as a function of irradiation frequency for a spin system in a high field according to spin-temperature theory is provided in Figure 3.
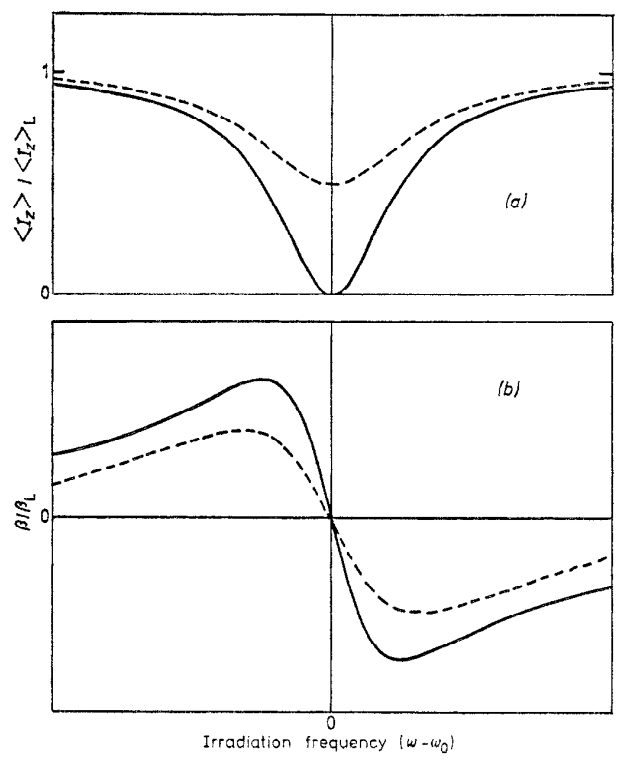

Figure 3. Variation of polarization (a) and of dipolar inverse temperature (b) as a function of irradiation frequency for a spin system in high field according to spin-temperature theory. Full curves: complete saturation, broken curves - partial saturation. (From Reference [9] @ (1978) IOP Publishing. Reproduced with permission. All rights reserved). 


\subsubsection{Fundamentals of the Optically Induced DNP}

Photoexcitation of electrons in a semiconductor under irradiation with unpolarized light (the case of circular polarization is considered in Appendix) produces an equal number of electrons with spin up and down. After that, the Overhauser effect [16] occurs on ${ }^{29} \mathrm{Si}$ nuclei in the bulk crystalline silicon (c-Si): the dynamic enhancement of the nuclear polarization, obtained by maintaining the electronic polarization off its equilibrium value. The essence of the Overhauser effect consists in the following.

A metal sample is placed in a constant magnetic field $\mathcal{B}_{0}$, which creates the polarization of electron spins. The electron energy in this field $E=\epsilon_{F}-\mu_{e} \cdot \mathcal{B}_{0}$, is expressed in terms of $\epsilon_{F}$ is the Fermi energy $\epsilon_{F}$ and the magnetic moment of the electron spin $S: \mu_{e}=-g_{e} \mu_{B e} S$, where $\mu_{B e}=\frac{e \hbar}{2 m_{e}}$ is the Bohr magneton and $g_{e}$ is the electron $g$-factor ([17], p. 420). It has two eigenvalues

$$
E_{j}=\epsilon_{F} \pm \mu_{B e} \mathcal{B}_{0}, j=\uparrow, \downarrow \text {, }
$$

where $g_{e}$ is taken as 2 . The electron spins tend to be aligned with the field. The resulting difference in spatial density of electrons with spins down and up $D \equiv N_{\downarrow}-N_{\uparrow}$ is selected as a measure of the electron spin magnetization $S_{z} \equiv \mu_{B e} D$. In thermal equilibrium under relatively weak magnetic fields, the value of $D$ takes on the form ([17], pp. 421 and 435)

$$
D_{0}= \begin{cases}\frac{3 N \mu_{B \in} \mathcal{B}_{0}}{2 \epsilon_{F}} & \text { at low lemperatures; } \\ \frac{N \mu_{B e} \mathcal{B}_{0}}{k T} & \text { at high temperatures. }\end{cases}
$$

The metal sample is subjected, furthermore, to a perpendicular alternating magnetic field (in particular, of the microwave radiation) $\mathcal{B}_{a c}=2 \mathcal{B}_{1} \cos (\omega t)$, whose frequency satisfies the electron spin resonance (electron paramagnetic resonance) criterion ([17], p. 488)

$$
\hbar \omega=2 \mu_{B e} \mathcal{B}_{0},
$$

where $2 \mu_{B e} \mathcal{B}_{0}$ is the distance between the eigenenergies given by Equation (1). $2 \mu_{B e} \mathcal{B}_{0} / \hbar$ is the precession frequency for the electron spin magnetic moment $\mu_{e}$ around the magnetic field $\mathcal{B}_{0}$. Such a radiation leads to the transitions between electronic states.

As a consequence, two kinds of processes take place in the sample-first, relaxation drives the magnetization to its equilibrium value $S_{z 0} \equiv \mu_{B e} D_{0}$ with a typical spin relaxation time $T_{1}$; second, the alternating field induces more transitions with the photoinduced relaxation time $\tau$ from the state with a higher population and therefore leads to a decrease of the magnetization:

$$
\frac{d D}{d t}=-\frac{D-D_{0}}{T_{1}}-\frac{D}{\tau}
$$

In a stationary state, $\frac{d D}{d t}=1$, the both processes are counterbalancing each other, what gives rise to the steady-state magnetization

$$
\left\langle S_{z}\right\rangle=\frac{S_{z 0}}{1+T_{1} / \tau} .
$$

At strong alternating magnetic fields, the resonance is saturated, that is $D=0$. In the general case, a saturation parameter

$$
s=1-\frac{\left\langle S_{z}\right\rangle}{S_{z 0}}
$$

changes from 0 when there is no alternating magnetic field to 1 when it is strong. It is a function of the ratio $T_{1} / \tau$ only:

$$
s=\frac{T_{1} / \tau}{1+T_{1} / \tau} .
$$


The amplitude of the microwave field that is necessary to saturate the resonance is inversely proportional to the geometric mean value of the spin relaxation time $T_{1}$ and the transverse relaxation time $T_{2}$, which is determined by the integrated width of the resonance line $w$ through $w=\pi \hbar / T_{2}$ [16].

Using NMR of ${ }^{29} \mathrm{Si}$, the spin-lattice relaxation time was determined as a function of mobile carrier concentration (sample resistivity) [18]. $T_{1}$ was established to be inversely proportional to the carrier concentration for the high-conductivity samples, approaching an asymptotic value in the purer samples (Figure 4).

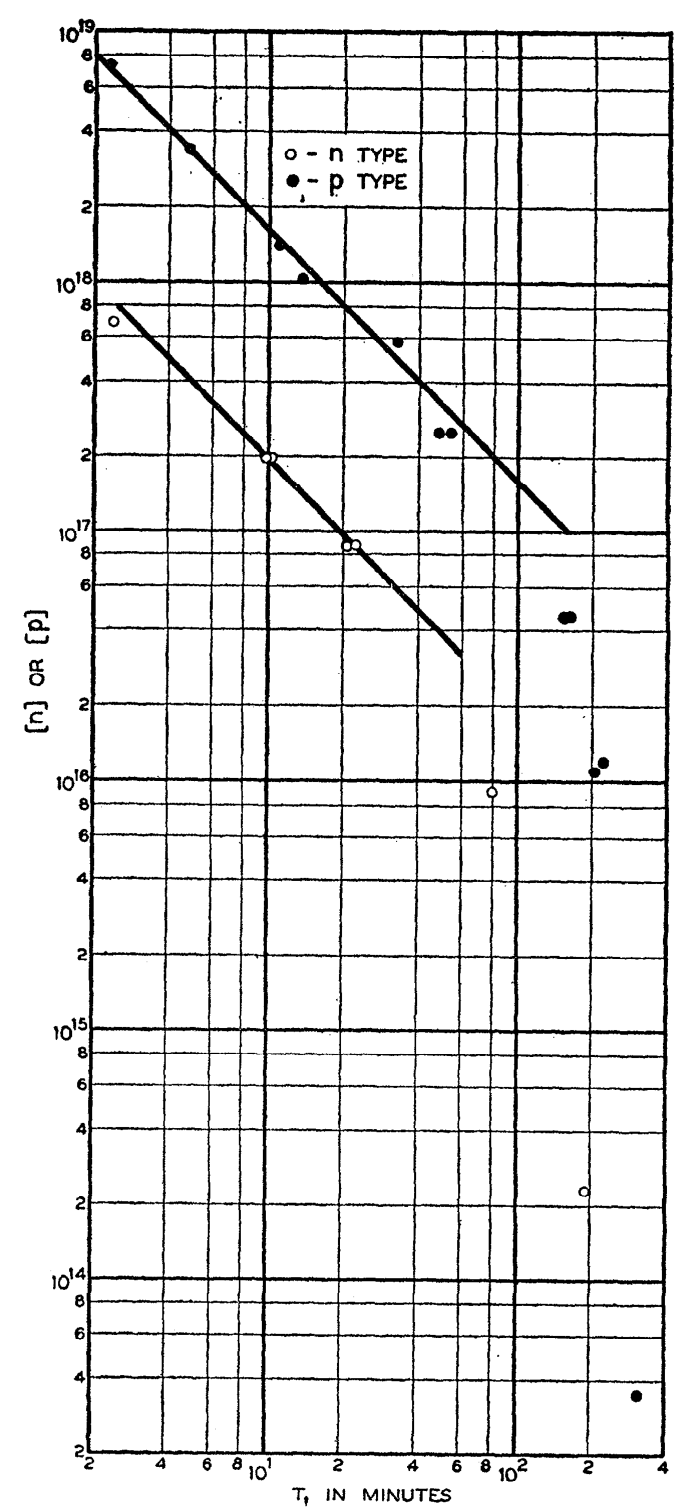

Figure 4. Plot of ${ }^{29} \mathrm{Si}$ spin-lattice relaxation times versus mobile carrier concentration. (Reprinted figure with permission from Reference [18] C (1956) by the American Physical Society).

Analysis of relaxation times in semiconductors [19] led to the conclusion that the relaxation of ${ }^{29} \mathrm{Si}$ in the exhaustion and intrinsic ranges should be accomplished by the hyperfine interaction between the nuclear spins and the spins of the mobile electrons or holes. However, there was a significant uncertainty in the density of mobile carriers. No differences were observed because of the crystal growth conditions (some crystals were pulled from quartz crucibles, some zone-refined without crucibles). The asymptotic values of $p$ - and $n$-type $\mathrm{Si}$ were substantially different. 
The low-field relaxation of nuclear hyperpolarization was studied in undoped and highly doped Si MPs at room temperature following removal from high field [20]. For nominally undoped particles (Figure 5a), two relaxation time scales were identified for ambient fields above $0.2 \mathrm{mT}$. The slower, $T_{1, \mathrm{~s}}$ was roughly independent of ambient field; the faster, $T_{1, \mathrm{f}}$, decreased with increasing ambient field. A model assumed the nuclear spin relaxation to occur at the Si MPs surface via a 2-electron mechanism in a good agreement with the experimental data in what concerns the magnetic-field independence of the slow relaxation time. For boron-doped particles (Figure 5b), a single relaxation time scale was observed. This suggested that for doped particles, mobile carriers and bulk ionized acceptor sites, rather than paramagnetic surface states, were the dominant relaxation mechanisms. Relaxation times for the undoped particles were not affected by tumbling in a liquid solution [20].

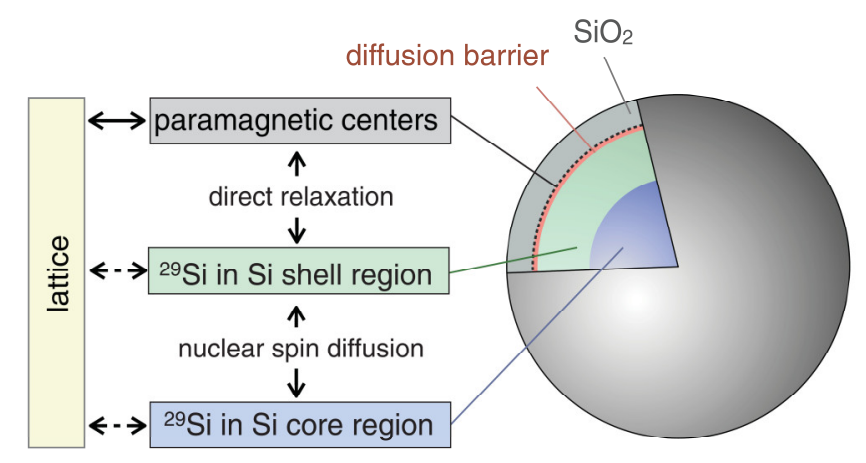

(a)

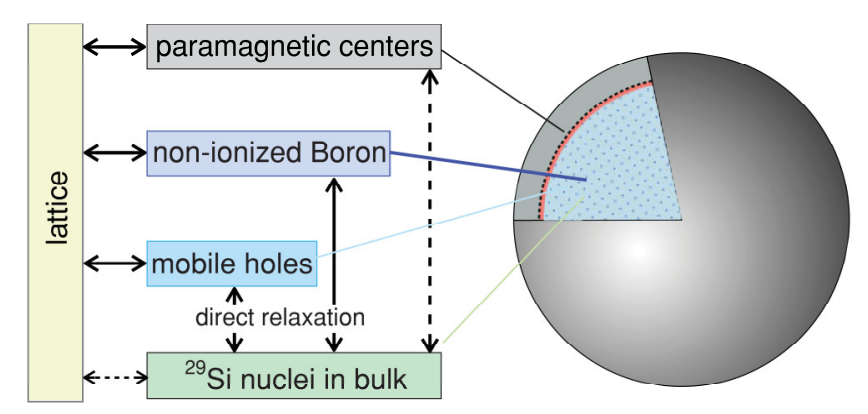

(b)

Figure 5. (a) Model showing the spin reservoirs and relaxation pathways in undoped silicon particles. Nuclei in the core region of the particle relax by transferring their magnetization to the nuclei in the shell region, nearer to the surface, by spin diffusion. The nuclei near the surface can relax quickly due to strong dipolar coupling to paramagnetic defects at the $\mathrm{Si} / \mathrm{SiO}_{2}$ interface. (b) Rate model showing dominant nuclear relaxation pathways of direct relaxation on mobile holes and short diffusion to non-ionized B (i.e., bound holes) throughout the bulk of the boron-doped particle. Long-distance spin diffusion to surface impurities is relatively slow and direct coupling of the nuclei to the lattice is weak. (Reprinted figures with permission from Reference [20] @ (2011) by the American Physical Society).

Nuclear polarization is most efficiently performed via the hyperfine interaction with electrons localized in a donor impurity [21]. The key issue is the hyperfine dipolar interaction between the magnetic moments of electron $\mu_{e}$ and nucleus $\mu_{n}=\mu_{B n} g_{n} I\left(\mu_{B n}=\frac{e \hbar}{2 m_{n} C}\right)$ separated in space by a vector $r$

$$
H=-\frac{8 \pi}{3} \mu_{e} \cdot \mu_{n} \delta(r)
$$


Here, $I$ and $\mu_{B n}$ (for nuclei with $I=\frac{1}{2}$ ) are the nuclear spin angular moment and nuclear Bohr magneton, respectively. As shown in Figure 6, the action of the hyperfine interaction is twofold. (i) Every nucleus is subjected, next to the applied magnetic fields, to a magnetic field of polarized electron spins fluctuating due to the aforementioned processes of spin relaxation. The magnitude of this field is proportional to the average (over the fluctuations) electron spin. The nuclear spin relaxation time due to the hyperfine interaction is $T_{n}^{\prime}$ (ii) Every electron is subjected, next to the applied magnetic fields, to the total magnetic field of all polarized nuclei. These two magnetic fields are comparable because electrons at low energy levels interact with a large number of nuclei. The electron spin relaxation time due to the hyperfine interaction is $T_{1}^{\prime}$. As a consequence, there occurs a feedback, which leads to a steady-state polarization of both electron and nuclear spins. There has been a renewed interest in the hole-nuclei hyperfine interaction in semiconductor quantum dots [22]. The main effect arises from the coupling of the nuclear spin with the hole wavefunction within the same unit cell, that is, the short-range part of the dipole-dipole interaction [12]. The potential effect of large inhomogeneous elastic strain in nanostructures (like quantum dots) on the microscopic Bloch hole wave function is considered as a possible route to engineer the hyperfine coupling.

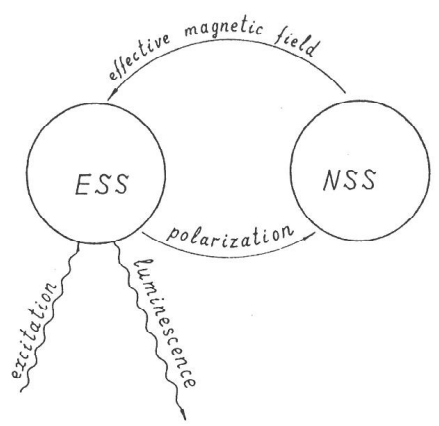

Figure 6. Feedback coupling of the electron spin system (ESS) and the nuclear spin system (NSS) in a semiconductor. Optically oriented electrons polarize the nuclei (arrow polarization) Because of the polarization, an effective magnetic field arises, which, in its turn, affects the electron orientation and hence the luminescence polarization. (Reprinted from Reference [21], (C) (1984), with permission from Elsevier).

Similar to the electron spins, the difference in numbers of nuclei (with spin $I=1 / 2$, like ${ }^{29} \mathrm{Si}$ ) possessing spins up and down $=M_{\uparrow}-M_{\downarrow}$ is selected as a measure of the nuclear spin magnetization $I_{z} \equiv \Delta / 2$. At arbitrary value of the nuclear spin $I, \Delta$ denotes the difference between the populations of the adjacent nuclear spin states. The total number of nuclear spins is supposed to be equal to $N=M_{\uparrow}+M_{\downarrow}$. (or the sum of all available spin eigenvalues at arbitrary $I$ ). The thermal equilibrium value of the difference $\Delta$ at $I$ is the fo the nuclear spin value llowing: [23]

$$
\Delta_{0}=\frac{N \mu_{B n} \mathcal{B}_{0}}{I(2 I+1) k_{B} T} .
$$

The corresponding steady-state magnetization on nuclei with spin $I=1 / 2$ is $I_{0}=\Delta_{0} / 2$. In terms of the gyromagnetic ratios, that is, $\gamma_{e}=-\frac{\mu_{B e}}{1 / 2}, \gamma_{n}=\frac{\mu_{B n}}{I}$, the relative magnetization of nuclei takes on the form (see Section IX C in Reference [12]):

$$
\frac{\left\langle I_{z}\right\rangle}{I_{0}}=1-s \frac{\left|\gamma_{e}\right|}{\gamma_{n}} .
$$

In analogue to the Overhauser effect [16], the steady-state nuclear polarization is enhanced as if the nuclear gyromagnetic ratio $\gamma_{n}$ would be increased by $s\left|\gamma_{e}\right|$. Near the saturation, when $s$ is close to 1 , this increase theoretically may be as large as $\left|\gamma_{e}\right| / \gamma_{n}=3310 \mathrm{in}^{29} \mathrm{Si}$ [17], (p. 489). The experimentally observed nuclear spin polarization enhancement extrapolated to an infinite time of continuous irradiation by an 
unpolarized light constitutes $\left\langle I_{z}\right\rangle / I_{0}=-5.6$ [14]. According to Equation (10), this implies the saturation parameter $s \sim 2 \times 10^{-3}$.

The nuclear magnetization (Figure 2a) produced by optical pumping in a field of $1 \mathrm{G}$ (extrapolated to infinite time of continuous irradiation) corresponded to the equilibrium polarization in a field of $28 \mathrm{kG}$ at $77 \mathrm{~K}$ and constituted $\left\langle I_{z}\right\rangle=3.7 \times 10^{-6}$. Thus, the enhanced nuclear polarization as high as $2.8 \times 10^{4}$ due to the optical pumping was experimentally demonstrated [14].

Polarized electronic spins are produced by optical pumping with circularly polarized light. Both spin directions are created in the conduction band. However, the unequal proportions $G_{+}$and $G_{-}$of transitions to spin-up and spin-down states lead to a net electronic polarization much larger than its equilibrium value (see Equation (VI.7) in Reference [15]):

$$
\left\langle I_{z}\right\rangle=I_{0}+\frac{T_{1} /}{1+T_{1} /}\left(\frac{G_{+}-G_{-}}{2}-S_{0}\right)
$$

where the first term in the parentheses is due to the optical pumping, while the second one is the saturation term [14]. As long as under optical pumping the latter is much smaller than the former and $I_{0} \ll\left\langle I_{z}\right\rangle$ and taking into account that $\frac{T_{1}}{\tau} \ll 1$ (it is typically $2 \times 10^{-3}[14]$ ), the steady-state nuclear spin polarization turns to

$$
\left\langle I_{z}\right\rangle \approx \frac{T_{1}}{\tau} \frac{G_{+}-G_{-}}{2} .
$$

It depends on matrix-element ratios describing electronic transitions between valence and conduction bands, as well as on electronic recombination and relaxation rates. Therefore, for this mechanism the value of the obtained nuclear spin polarization is of key importance rather than the enhancement of the relative nuclear spin polarization. A direct measurement of $T_{1} / \tau$ performed in Reference [14] demonstrated that only $0.4 \%$ was achieved of the magnetization that could be expected if only one kind of electronic spin had been created. This was due to the matrix elements between the valence and conduction bands in $\mathrm{Si}$, which is an indirect bandgap material.

A theory proposed in Reference [24] established the first link between the degrees of spin polarization of electrons and the circular polarization for each of the dominant phonon-assisted optical transitions, which was crucial for the subsequent development of applications. A method of hyperpolarizing Si MPs and NPs demonstrated an increase of the ${ }^{29} \mathrm{Si}$ MR signal by up to $3-5$ orders of magnitude via the enhanced nuclear spin alignment, while retaining this improved signal for tens of minutes $[25,26]$.

\subsubsection{Optical Spin Orientation in Various Semiconductors}

Since the first successful experiment [14], discussed in the previous Subsection, there has been a steady interest in theoretical and experimental investigation of optical spin orientation in various semiconductors. Among them, c-Si is an advantageous material for spintronics because of its long spin relaxation time and dominating technology. The dynamic polarization of ${ }^{29} \mathrm{Si}$ nuclei in phosphorus-doped $\left(N_{\mathrm{d}}=1.5 \times 10^{13} \mathrm{~cm}^{-3}\right) n$-type c-Si induced by optical pumping was investigated in Reference [27]. During illumination of c-Si, the magnetization of ${ }^{29} \mathrm{Si}$ nuclei becomes gradually established, following an exponential law with a time constant equal to the spin-lattice relaxation time $T_{1}$ of ${ }^{29} \mathrm{Si}$ nuclei. Limiting relative polarization of the ${ }^{29} \mathrm{Si}$ nuclei obeyed the following equation [27]:

$$
\frac{\left\langle I_{z}\right\rangle}{I_{0}}=1-s \frac{\left|\gamma_{e}\right|}{\gamma_{n}} \frac{n_{p h}}{n_{0}+n_{p h}} .
$$

With the concentrations $n_{\mathrm{ph}}$ of photoexcited and $n_{0}$ of thermal electrons, correspondingly. Without light, the nuclear magnetization equaled its equilibrium value. When the concentrations of photoexcited electrons was much higher than that of thermal electrons, the saturation value was described by Equation (13) in agreement with experiment (see Figure 7). The limiting degree of nuclear polarization 
was determined by the spin polarization of both the photoexcited and thermal electrons in the conduction band. The presence of rare earth impurities (Gd) in combination with shallow impurities (P) resulted in increased optical pumping efficiency, because the rare earth impurities increased the ionization energy of the shallow impurities [28]. More recently, the dynamic-nuclear-polarization capabilities of optical pumping at $4 \mathrm{~K}$ and a magnetic field of $7 \mathrm{~T}$ were investigated with a ${ }^{29} \mathrm{Si}$-enriched sample. Using NMR spectroscopy, the ${ }^{29} \mathrm{Si}$ polarization as high as up to $0.25 \%$ was demonstrated [29]. The investigations of above-bandgap excitations look promising for further enhancement of ${ }^{29} \mathrm{Si}$ polarization in the sample area close to the exposed surface.

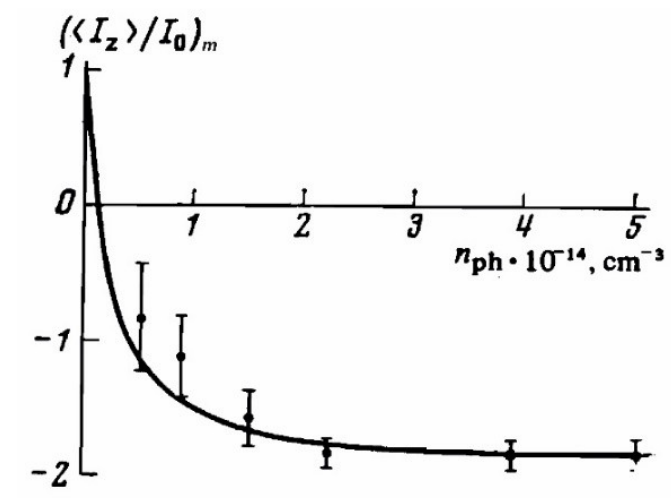

Figure 7. Limiting relative polarization $\left(\frac{\left\langle I_{z}\right\rangle}{I_{0}}\right)_{m}$ of the ${ }^{29} \mathrm{Si}$ nuclei as a function of the concentration $n_{\mathrm{ph}}$ of photoexcited electrons in $n$-type Si irradiated with unpolarized light. (Reproduced from Reference [27], with the permission of American Institute of Physics).

However, c-Si is an indirect bandgap semiconductor and it was soon clear that higher nuclear polarizations could be generated in direct bandgap materials. The main focus of solid-state optical pumping therefore shifted to direct bandgap semiconductors soon after the pioneering experiment of Lampel [14], which was analyzed in Section 2.1.3. The stationary spin orientation of photoelectrons excited by circularly polarized light in $\mathrm{Ga}_{x} \mathrm{Al}_{1-\mathrm{x}} \mathrm{As}$, as detected by the degree of the circular polarization of photoluminescence, was determined by the value of the nuclear magnetization [30]. The optical method manifested a very high sensitivity (signal-to-noise ratio of the order of 100:1 when the total number of photoelectrons constituted $10^{9}$ ) and could be applied to samples at microscale. Optically-pumped, directly-detected NMR experiments on the bulk crystalline InP (c-InP) were performed [31] by using irradiation of a sample of undoped c-InP wafer from a diode laser to enhance the spin polarization of ${ }^{31} \mathrm{P}$ nuclei near the sample surface. The spin polarization was found to depend on the light polarization. The spin polarization magnitude and polarization transfer efficiency of the from the semiconductor substrate to the overlayer (Figure 8) were high enough to permit solid state NMR measurements on sub-nanomole quantities of molecules in the overlayer [31]. The optically induced dynamic nuclear polarization in CdS was observed by radio-frequency detection of the ${ }^{113} \mathrm{Cd}$ nuclear magnetic resonance signal for $T<2 \mathrm{~K}$. At this temperature, the optically induced spin polarization was negative when compared to the thermal polarization, irrespective of the helicity of the pumping light [32]. By increasing the depth of the donor levels (Figure 9), a very significant nuclear polarization was achieved because of the increase of the electron spin relaxation time. 


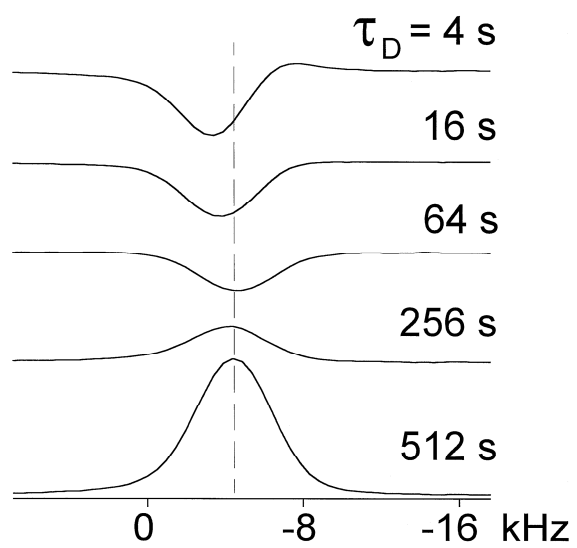

Figure 8. Dependence of the ${ }^{31} \mathrm{P}$ optically-pumped nuclear magnetic resonance (NMR) lineshape on the period during which the shutter is closed $\tau_{D}$, with the period during which the shutter is open $\tau_{L}=120 \mathrm{~s}$. At short $\tau_{D}$, the lineshape is dominated by signal from the optically-pumped surface-layer. At long $\tau_{D}$, the lineshape is dominated by signal from the bulk of the InP sample which increases due to spin-lattice relaxation. (Reprinted from Reference [31], (C) (1998), with permission from Elsevier).

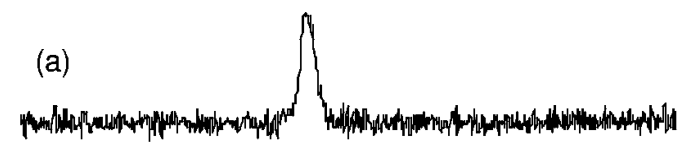

(b)

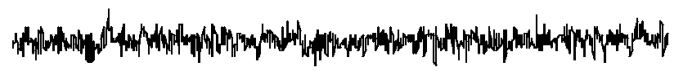

(c)

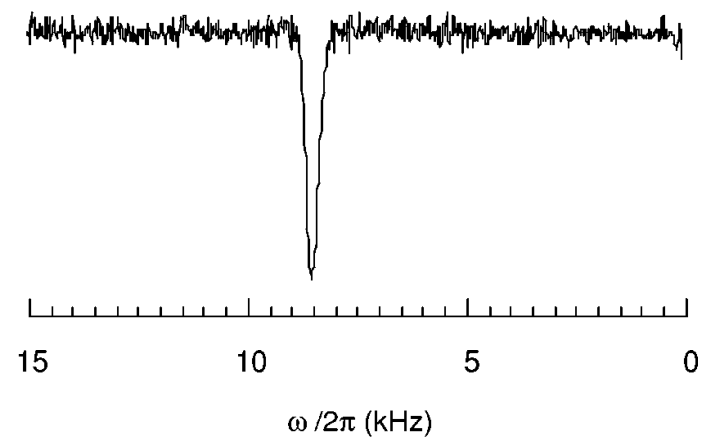

Figure 9. ${ }^{113} \mathrm{Cd}$ NMR spectra of the high-resistivity CdS single crystal recorded at $2 \mathrm{~K}$. The Boltzmann signal is shown in (a), the optically enhanced signal after an irradiation time $\tau_{L}=5600 \mathrm{~s}$ at $2.60 \mathrm{eV}$ with circularly polarized light and $520 \mathrm{~mW}$ laser power (c). The control measurement (b) was obtained under the same conditions as (c) but with the shutter closed during $\tau_{L}$. (Reprinted figure with permission from Reference [32] (C) (1999) by the American Physical Society).

The mechanism of optical pumping can be considerably more complicated and less well understood. Both contact hyperfine and dipolar hyperfine interactions can contribute appreciably to cross-relaxation. The spin polarization of the photoexcited electrons may depend on the pumping wavelength and the magnetic field. The precise nature of the photoexcitation that dominate optical pumping, which are expected to be localized excitations, may be unknown. The roles of photogenerated holes, doped carriers and radiationless transitions to a metastable excited state after the initial absorption of a photon are to be clarified [31].

A theory of the optical spin orientation under indirect optical injection of carriers and spins in bulk Si, proposed in Reference [33], predicted the maximum degree of spin polarization as high as $25 \%$ at low temperatures and $15 \%$ at high temperatures. These values of the degree of the spin polarization implied the term "optically induced spin hyperpolarization" for the phenomenon, which is 
the main objective of the present review. The theory of Reference [33] was immediately applied to analyze two-photon indirect optical injection of carriers and spins and two-color coherent control in bulk Si [34]. Measurements of the electron spin lifetime and optical orientation efficiency were also carried out in Ge by using electrical detection of radio frequency modulated spin polarization [35]. The optical spin injection and spin lifetime in Ge heterostructures were experimentally investigated in Reference [36]. Th valley-dependent spin polarization and long-lived electron spins were detected in Ge [37]. Spin-dependent optical properties were investigated as a function of strain in Si and Ge [38]. Theory of spin-dependent intravalley and intervalley electron-phonon scatterings was developed for Ge [39]. The optical spin-selection rules in bulk Si and Ge/Si quantum dots were experimentally unveiled in Reference [40]. Two optical routes of cold carrier injection in Si were revealed by time-resolved excitation spectroscopy [41]. The optical generation of pure spin currents was detected at the indirect gap of bulk Si [42]. Hot phonon and carrier relaxation in Si single crystalline wafers with the (100) crystallographic orientation were determined by transient extreme ultraviolet spectroscopy [43].

The spin-to-charge conversion for hot photoexcited electrons was experimentally detected in Ge [44]. The successful optical generation of a net spin polarization of electrons in the strained $\mathrm{Ge}_{1-\mathrm{x}} \mathrm{Sn}_{\mathrm{x}}$ alloy, which manifests hybridization of the spin properties of Ge with the peculiar electronic states offered by $\alpha$-Sn, was demonstrated by recording PL spectra, the optical spin orientation being robust over a wide temperature range up to room temperature [45]. The spin coherence time of photoexcited spin-polarized electrons was found to decay from $12 \mathrm{~ns}$ to $6 \mathrm{~ns}$ when increasing the magnetic field from 0 to $53.5 \mathrm{mT}$ owing to $g$-factor fluctuations as long as spin dephasing was triggered by the cooling process, during which optically generated electrons underwent momentum relaxation under random effective magnetic fields due to local changes in the alloy composition and strain [45].

\subsection{Microwave-Irradiation-Induced Nuclear Polarization}

\subsubsection{Microwave-Induced Optical Nuclear Polarization}

The hyperpolarization of ${ }^{29} \mathrm{Si}$ nuclear spins is usually generated by solid-state DNP at low temperatures and high magnetic fields to spin-polarize an electron to near unity. This spin polarization is then transferred to nearby nuclear spins through microwave-mediated dipolar interactions, similar to those occurring in the liquid phase with ${ }^{13} \mathrm{C}[46]$.

A combination of the DNP and the photoexcitation of the electron-pair spin to the triplet state is known as microwave-induced optical nuclear polarization [47]. The polarization from the electron triplet states is transferred via microwaves to the nuclear spins. The hyperpolarization of electron spins can be prepared at low magnetic fields, since it is generated due to selectivity in crossing from excited singlet states to hyperpolarized triplet states. Because the triplet states have a longer relaxation time as compared to the singlet state, the hyperpolarized nuclear spins can be generated by DNP at room temperature. Feasibility of the nuclear hyperpolarization at low magnetic field and room temperature provides an advantage to microwave-induced optical nuclear polarization as compared to the DNP. The unpaired electron spins do not perturb the NMR signals because the decay time of the triplet state to the diamagnetic state is shorter than the relaxation time of the longitudinal nuclear magnetization [47]. While the microwave-induced optical nuclear polarization was demonstrated for organics as fluorene $\mathrm{C}_{13} \mathrm{H}_{10}$ crystals [48], it seems to be a possible mechanism for inorganic semiconductor systems as NP/MPs with nc-Si, where triplet excitonic state are rather probable (see Section 3).

\subsection{2. ${ }^{29} \mathrm{Si}$ Surface-Enhanced NMR Spectroscopy}

Fast characterization of the distribution of surface bonding modes and interactions in a series of functionalized materials was achieved via surface-enhanced nuclear magnetic resonance spectroscopy using DNP [49]. Surface-enhanced ${ }^{29}$ Si DNP NMR spectra were obtained by using incipient wetness impregnation of the sample with a solution containing a polarizing radical (TOTAPOL). The bonding 
topology of functional groups was identified in materials obtained via a sol-gel process and in materials prepared by post-grafting reactions [49].

The remarkable gain in time provided by surface-enhanced NMR Spectroscopy using DNP, which is of the order of 400, allows for a facile acquisition of 2D correlation spectra for characterization of the distribution of the surface bonding modes/interactions (Figure 10) in functionalized materials [49].
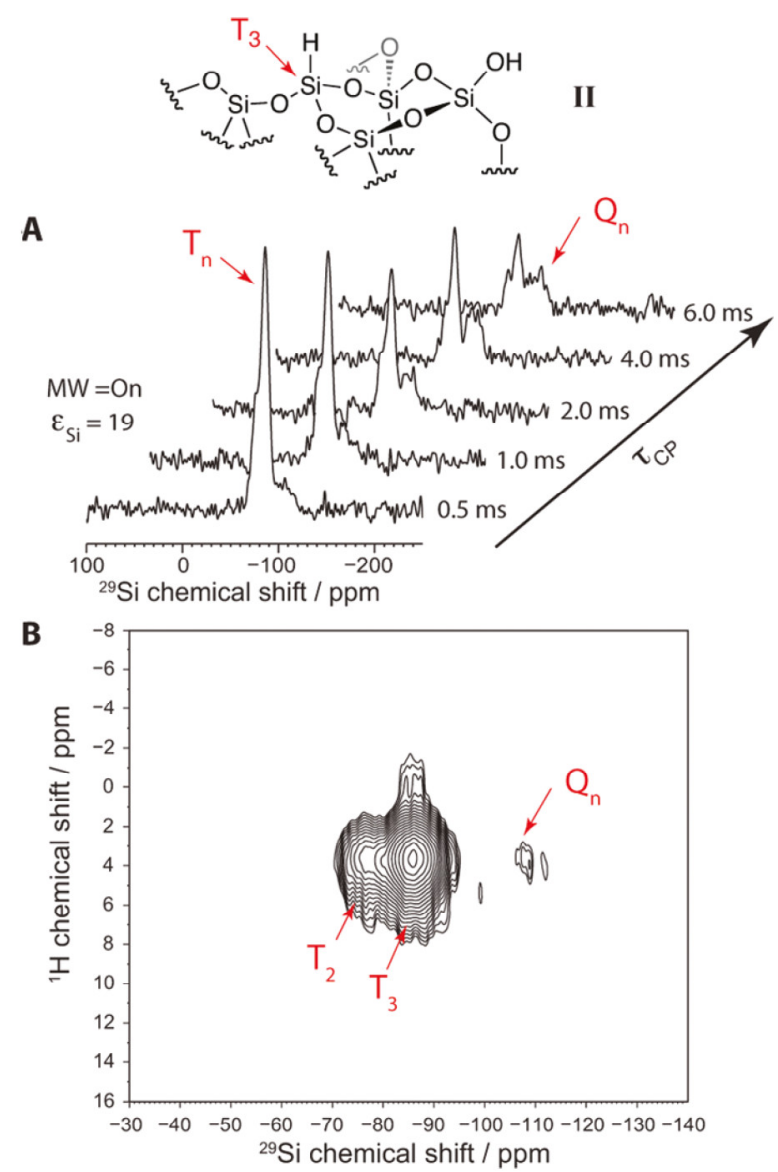

Figure 10. (A) Dynamic nuclear polarization (DNP)-enhanced ${ }^{29}$ SiCross-Polarization Magic Angle Spinning (CPMAS) NMR spectra of II as a function of the CP mixing time $\tau_{\mathrm{CP}}$. Spectra were recorded with 16 scans. (B) Contour plot of a two-dimensional ${ }^{1} \mathrm{H}-{ }^{29} \mathrm{Si}$ spectrum of II recorded with DNP. $64 t_{1}$ increments of $64 \mu$ s with eight scans each were recorded with $\tau_{C P}=1.0 \mathrm{~ms}$. Total experimental time was $8.5 \mathrm{~min}$. (Reprinted with permission from Reference [49]. (C) 2011 American Chemical Society).

\subsubsection{Long $T_{1}$ Nanoparticles for Hyperpolarized ${ }^{29} \mathrm{Si}$ MRI}

The synthesis, materials characterization and DNP of amorphous and crystalline Si NPs for use as hyperpolarized MRI agents were represented in Reference [26]. The Si NPs of $\sim 10 \mathrm{~nm}$ size were synthesized by a metathesis reaction and surface functionalized with a variety of passivating ligands. Because of the long size-adjusted nuclear spin-lattice relaxation time $T_{1}>600 \mathrm{~s}$ (Figure 11), Si NPs can be hyperpolarized by low-temperature DNP (Figure 12) and retain the polarization for $>300 \mathrm{~s}$ [26]. 


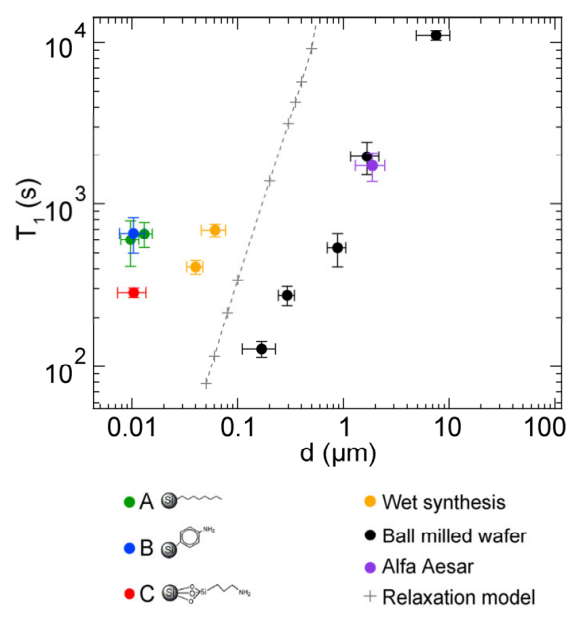

Figure 11. Nuclear spin relaxation $\left(T_{1}\right)$ times at $2.9 \mathrm{~T}$ and $300 \mathrm{~K}$ as a function of particle diameter $\mathrm{d}$ for $\mathrm{Si}$ NPs. Previously reported $T_{1}$ measurements of other Si particles are shown as a reference. Simulations of the nuclear relaxation based on a spin diffusion model are also shown. Vertical error bars are from exponential fits to relaxation data; horizontal error bars are from size distributions. (Reprinted with permission from Reference [26]. (C) 2013 American Chemical Society).

(a)

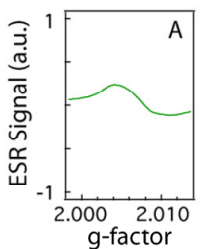

(b)

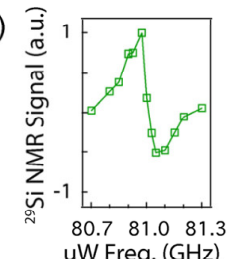

(c)

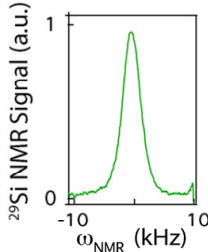

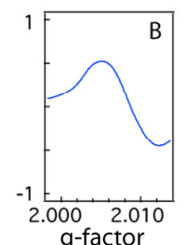
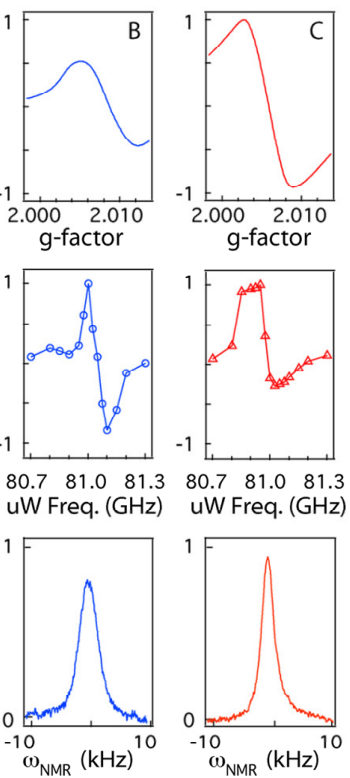

Figure 12. Electron spin resonance and DNP of (A) octyl-, (B) 4-aminophenyl- and (C) APTMS-terminated Si NPs. (a) Electron spin resonance signal and (b) ${ }^{29} \mathrm{Si}$ nuclear polarization as a function of microwave frequency under DNP conditions. (c) ${ }^{29} \mathrm{Si}$ NMR spectra obtained under DNP conditions. (Reprinted with permission from Reference [26]. (C) 2013 American Chemical Society).

In solid-state spin-1/2 materials, such as $\mathrm{Si}$, the primary source of nuclear spin-lattice relaxation is the interaction with unbonded electrons or holes, which occur in the form of free charge carriers or paramagnetic centers either at lattice defect sites or at the surface. In pure materials, the nuclear spin polarization is to a significant extent protected from sources of relaxation, resulting in bulk relaxation times of many hours [11,12,18]. A transition to the nanoscale resulted in spin relaxation times that depend on the particle size, purity and density of defects [11]. The colloidal synthesis of Si NPs with average diameters of $\sim 10 \mathrm{~nm}$ at room temperature provided spin relaxation times that exceed $600 \mathrm{~s}$. This resulted in fabrication of NPs that revealed significantly longer size-adjusted ${ }^{29}$ Si nuclear spin relaxation times $T_{1}$ than commercially available Si particles [11] and diamond NPs of similar sizes [50]. These Si NPs are the smallest characterized for hyperpolarization applications. Due to their size and 
long $T_{1}$ times, they are perspective as in vivo imaging agents, which are suitable for intracellular investigations and for crossing the blood-brain barrier [26].

\subsubsection{DNP in Si Microparticles}

High ${ }^{29} \mathrm{Si}$ spin polarization was achieved using DNP in microcrystalline Si powder [51]. Unpaired electrons in the powder are related to Si DBs in the amorphous region of microcrystalline grains. Nuclei in the amorphous region become polarized by forced electron-nuclear spin flips induced by off-resonant microwave radiation (Figure 13). Nuclei in the crystalline region are polarized by spin diffusion across crystalline boundaries. Hyperpolarized Si MPs have long $T_{1}$ (Figure 14) and hence can be used as tracers for MRI [51].

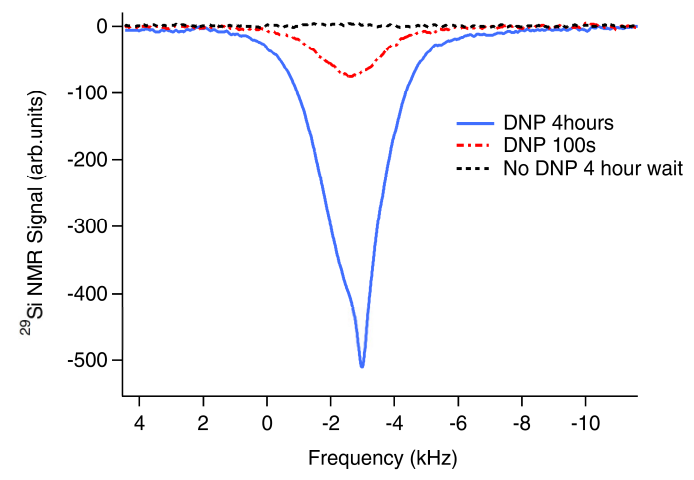

Figure 13. ${ }^{29} \mathrm{Si}$ NMR spectra acquired at $1.4 \mathrm{~K}$ for different microwave irradiation times in $B=2.35 \mathrm{~T}$ $\left(f_{0}=19.89 \mathrm{MHz}\right)$. The spectrum acquired without the microwave irradiation is shown using the dashed line. (Reprinted figure with permission from Reference [51] ( ) (2008) by the American Physical Society).

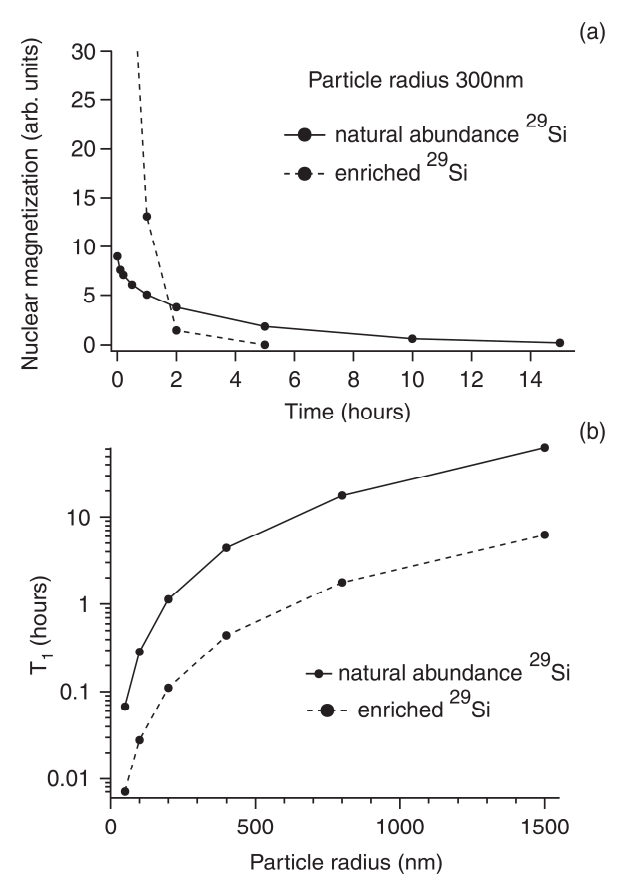

Figure 14. (a) Simulations of the ${ }^{29} \mathrm{Si}$ magnetization decay due to spin diffusion and instantaneous relaxation at the surface for particles with $300 \mathrm{~nm}$ radius. (b) Simulated $T_{1}$ relaxation time as a function of the particle size. Solid line is for the naturally abundant ${ }^{29} \mathrm{Si}$ and dashed line is for the ${ }^{29} \mathrm{Si}$ enriched sample. (Reprinted figure with permission from Reference [51] @ (2008) by the American Physical Society). 


\subsubsection{In Vivo MRI of Hyperpolarized Si Particles}

Natural physical properties of Si (Figure 15) provide surface electronic states for DNP, long depolarization times, insensitivity to the in vivo environment and surfaces favorable for functionalization. In vivo imaging of hyperpolarized ${ }^{29} \mathrm{Si}$ in Si MPs and NPs by MRI is perspective for applications to gastrointestinal, intravascular and tumor perfusion imaging at sub-picometer concentrations (Figure 16) [25].
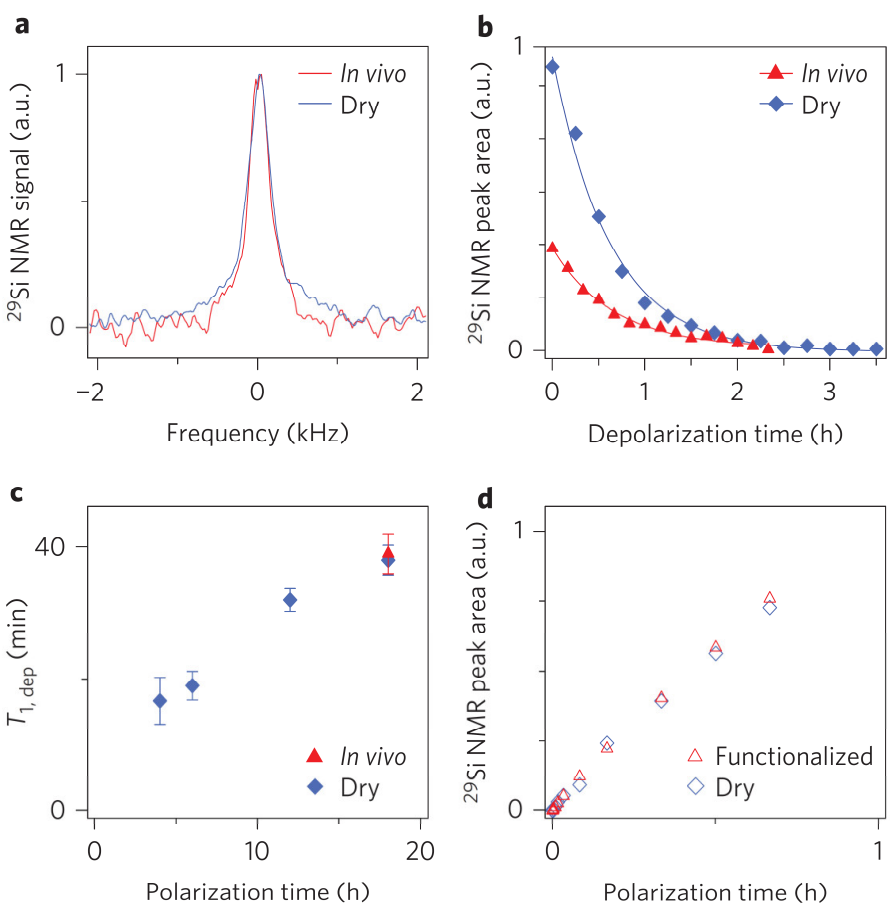

Figure 15. ${ }^{29}$ Si nuclear spin dynamics in silicon particles. (a) ${ }^{29} \mathrm{Si}$ NMR spectrum of hyperpolarized particles recorded in vivo and dry at $30 \mathrm{~min}$ delay. (b) Decay of ${ }^{29} \mathrm{Si}$ nuclear polarization in hyperpolarized Si particles recorded in vivo and dry. Both plots show a characteristic decay of $T_{1 \text {,dep }} \approx 40 \mathrm{~min}$ for hyperpolarization time $\tau_{\mathrm{pol}}=8 \mathrm{~h}$ using variable flip angle acquisition. The difference in absolute signal is mainly due to the reduced concentration within the active region of the coil. (c) Characteristic ${ }^{29} \mathrm{Si}$ depolarization time constants $\left(T_{1, \mathrm{dep}}\right)$ in dry particle phantoms recorded in the imager for polarization times of 4, 6, 12 and $18 \mathrm{~h}$. (d) Time evolution of the ${ }^{29} \mathrm{Si}$ nuclear polarization under DNP conditions for particles with and without surface functionalization with polyethylene glycol. The signal is normalized by sample weight. (Reprinted by permission from Springer Nature [25], (C) (2013)).
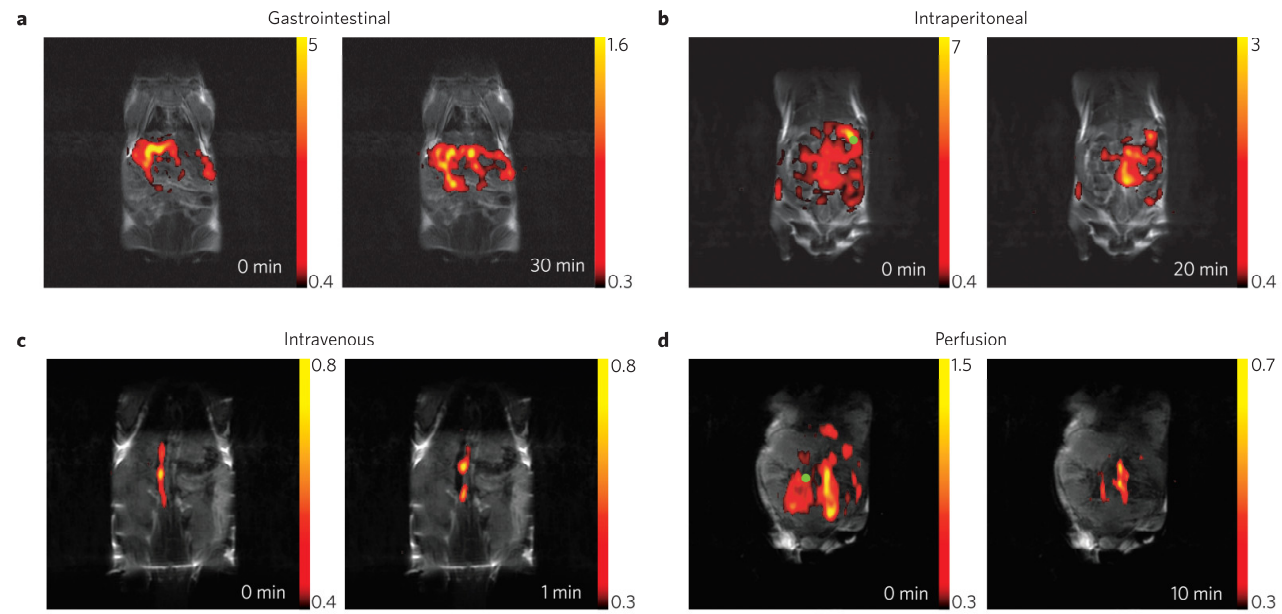
Figure 16. In vivo applications of ${ }^{29} \mathrm{Si}$ MRI using hyperpolarized Si MPs. (a-c) In vivo co-registered ${ }^{1} \mathrm{H}:{ }^{29} \mathrm{Si}$ MRI of hyperpolarized Si particles administered intragastrically (a), intraperitoneally (b) and intravenously (c) via a tail-vein catheter. (d) Demonstration of perfusion imaging using ${ }^{29} \mathrm{Si}$ MRI of hyperpolarized Si MPs injected into the tumor of a prostate cancer (TRAMP) animal. In panels (b) and (d), the green circle shows the approximate location of the catheter. The color scale bars show ${ }^{29} \mathrm{Si}$ signal intensity in arbitrary units. (Reprinted by permission from Springer Nature [25], (C) (2013)).

\subsubsection{Si Nanoparticles for Hyperpolarized MRI}

MRI of hyperpolarized nuclei was shown to provide a very high image contrast with little or no background signal [11]. To date, in vivo applications of pre-hyperpolarized materials have been limited by relatively short nuclear spin relaxation times. Si NPs were proposed as a new type of hyperpolarized MRI agent. Nuclear spin relaxation times for a variety of Si NPs were found to be extremely long, ranging from many minutes to hours at room temperature (Figure 17), allowing hyperpolarized NPs to be transported, administered and imaged on practical time scales [11]. Additionally, Si NPs can be surface functionalized using techniques common to other biologically targeted NP systems. These results suggest that Si NPs can be used as a targetable, hyperpolarized MRI agent (Figure 18) with a large range of potential applications.

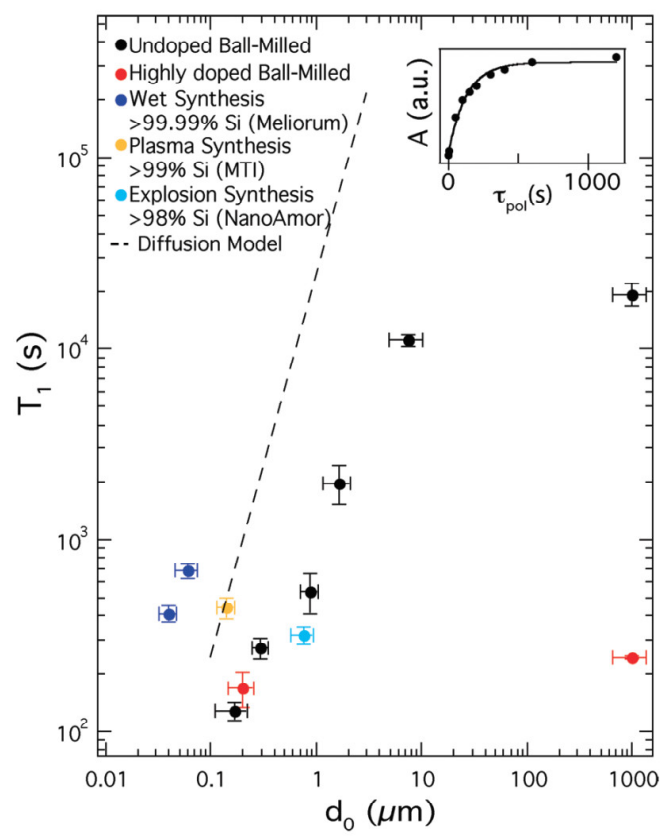

Figure 17. NMR Properties of Si particles. Nuclear spin relaxation $\left(T_{1}\right)$ times at $2.9 \mathrm{~T}$ as a function of particle diameter $d_{0}$ for various Si particles. Vertical error bars are from exponential fits to relaxation data; horizontal error bars represent the dispersion of size distributions. Inset: Fourier-transform NMR peak amplitude, A, as a function of polarization time for the ball-milled high-resistivity particles with $d_{0}=0.17 \mu \mathrm{m} . T_{1}$ values were measured using a saturation recovery spin echo pulse sequence. (Reprinted with permission from Reference [11]. (C) (2009) American Chemical Society). 

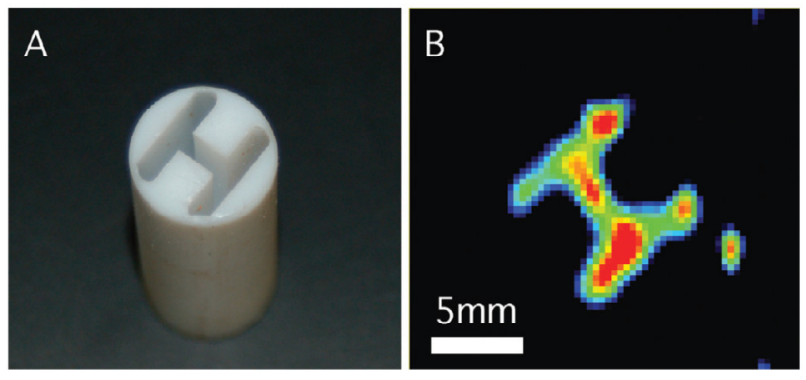

Figure 18. ${ }^{29} \mathrm{Si}$ MRI of hyperpolarized Si NPs. (A) H-shaped phantom filled with high-resistivity Si particles $\left(d_{0}=1.6 \mu \mathrm{m}\right)$ pre-polarized at low temperature $(T=4.2 \mathrm{~K})$ and high magnetic field $(B=5 \mathrm{~T})$ for $60 \mathrm{~h}$ and warmed and transferred to a $4.7 \mathrm{~T}$ imager. (B) single ${ }^{29} \mathrm{Si}$ image of phantom in (A). No ${ }^{29} \mathrm{Si}$ image could be obtained without hyperpolarization using the same sequence. (Reprinted with permission from Reference [11]. (C) (2009) American Chemical Society).

Micrometer-sized Si MPs, which reveal the longest known relaxation times up to 136 min at room temperature at $2.9 \mathrm{~T}$ [20], are difficult to administer and impossible to handle in-vivo. A new window for MRI applications was opened in Reference [52], where it was demonstrated that ${ }^{29} \mathrm{Si}$ NPs in powder synthesized from a gas phase using a laser-assisted technique, with average particle size of $55 \pm 12 \mathrm{~nm}$ can be hyperpolarized with superior properties, that is, maximal polarization about $12.6 \%$ with a long $T_{1}$ relaxation time of $42.3 \pm 0.1 \mathrm{~min}$ at room temperature (Figure 19A). Such a long depolarization time enabled the observation of ${ }^{29} \mathrm{Si} \mathrm{NMR}$ signal more than $5 \mathrm{~h}$ after transfer to the imaging system (Figure 19B,C). The presence of PEG polymer on the surface of the nanoparticles resulted in shortening of $T_{1}$ relaxation times at room temperature from $42.3 \pm 0.1 \mathrm{~min}$ to $34.0 \pm 0.3 \mathrm{~min}$ (Figure 19A), while no change in the polarization level was observed. A further significant increase in the polarization level is proposed by lowering the temperature (to $<2 \mathrm{~K}$ ) in the course of the DNP process, using a dedicated long-run helium bath or helium-free cryostat, boosting the obtainable ${ }^{29} \mathrm{Si}$ polarization. Conducting the DNP process at higher magnetic fields is also expected to increase final nuclear polarization. The limited availability of high-power microwave sources operating in the frequency range above $100 \mathrm{GHz}$ is presently the major difficulty.

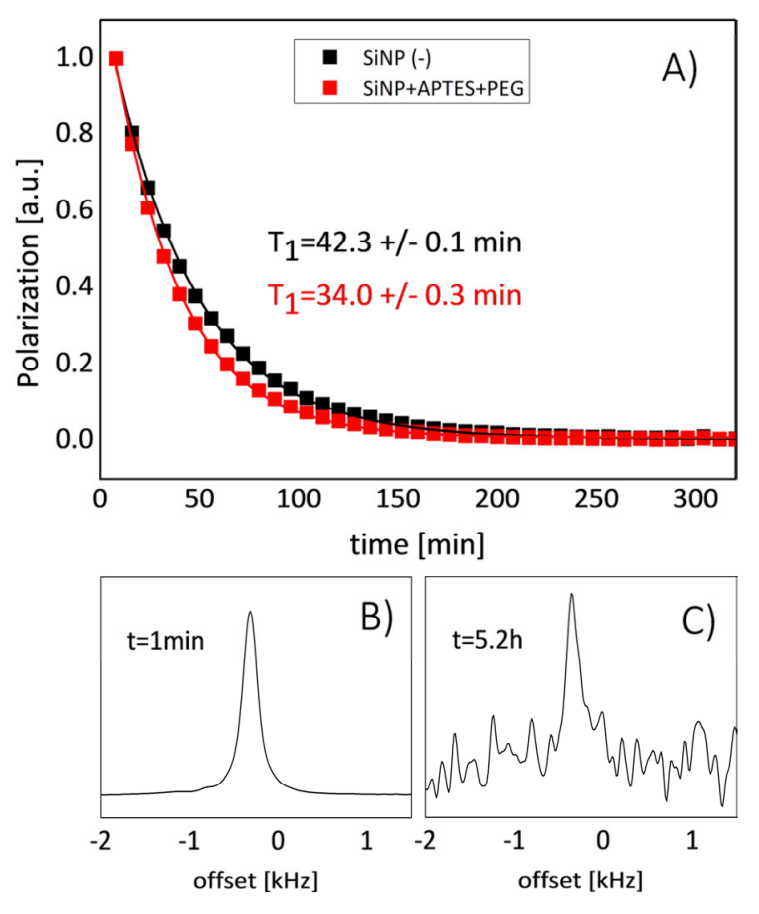


Figure 19. (A) Comparison of $T_{1}$ relaxation between pure (black) and PEG functionalized (red) silicon NPs. The Fourier-transformed free induction decay signal acquired at $t=1 \mathrm{~min}(\mathbf{B})$ and $t=5.2 \mathrm{~h}$ (C) at room temperature using a $9.4 \mathrm{~T}$ imaging system. (Reprinted from Reference [52], (C) (2017), Springer Nature. This article is licensed under a Creative Commons Attribution 4.0 International License http://creativecommons.org/licenses/by/4.0/).

Optimal physical and chemical conditions, including surface-defect concentration adjusted to the particle size, are necessary to achieve a high level of DNP enhancement [10]. Real-time MRI-guided catheter tracking using hyperpolarized ${ }^{29} \mathrm{Si}$ particles (polycrystalline/amorphous; average mean diameter $\sim 2 \mu \mathrm{m}$ ) was demonstrated [53]. The increased signal of the ${ }^{29} \mathrm{Si}$ particles is generated via low-temperature, solid-state DNP and the particles retain their enhanced signal for $\geq 40 \mathrm{~min}$, thus allowing imaging experiments over extended time durations in phantoms and live mice. The measured hyperpolarized relaxation rate of the ${ }^{29} \mathrm{Si}$ particles was $\sim 25 \mathrm{~min}$ at $7 \mathrm{~T}$ and room temperature.

A variety of particle sizes ( $20 \mathrm{~nm}$ to $2 \mu \mathrm{m}$ ) were found to have hyperpolarized relaxation times ranging from $\sim 10$ to $50 \mathrm{~min}[54,55]$. The addition of different functional groups to the $\mathrm{Si}$ particle surface was shown to provide no effect on the hyperpolarization buildup or decay rates and allowed for in vivo imaging over long time-scales. Hyperpolarized Si particles were demonstrated to be available agents for targeted, noninvasive and nonradioactive molecular imaging of various cancer systems [54] and a powerful theragnostic deep tissue imaging platform [55]. In vivo studies examined diverse particle administration routes in mice, including intraperitoneal injection, rectal enema and oral gavage [54]. Incorporating exogenous radicals (e.g., 2,2,6,6-tetramethyl-1-piperidinyloxy (TEMPO)) into Si NPs suspensions is proposed order to improve the characteristics of hyperpolarization of ${ }^{29} \mathrm{Si}$ nuclear spins to levels sufficient for in vivo MRI [56] (Figure 20). Without any added radical, $T_{1}$ values ranged from $\sim 23 \mathrm{~min}$ ( $30 \mathrm{~nm}$ particles) to $\sim 36 \mathrm{~min}$ ( $200 \mathrm{~nm}$ particles). This size-dependence of $T_{1}$ is largely expected given the spin-diffusion model [20] for the relaxation of core nuclei to the surface. At optimal TEMPO concentrations $(30-60 \mathrm{mM})$, the shortened $T_{1}$ values of the $\mathrm{Si}$ particles were overshadowed by the significant increase in the ${ }^{29} \mathrm{Si}$ MR signal [56].

a)

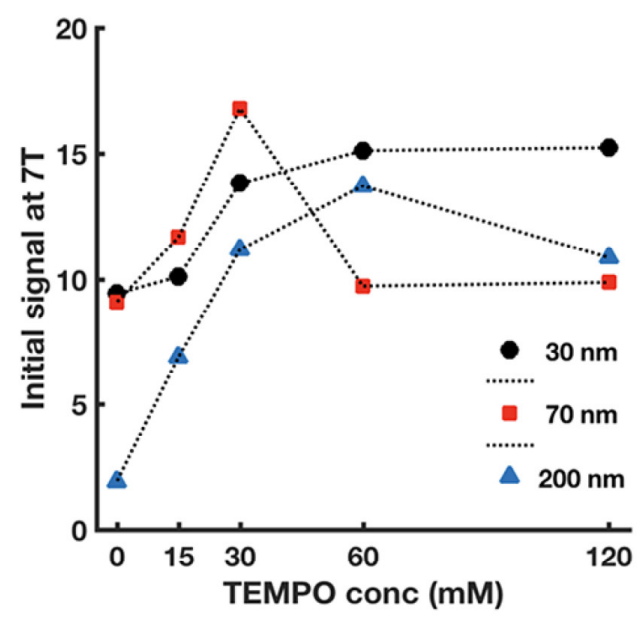

b)

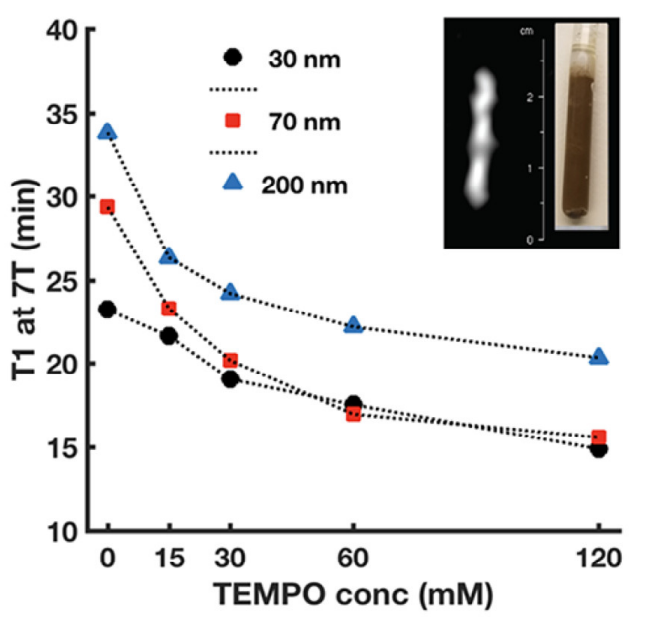

Figure 20. Optimization of TEMPO concentrations. (a) Calibration of TEMPO concentration vs ${ }^{29} \mathrm{Si}$ hyperpolarization level for Si NPs of different size: 30, 70 and $200 \mathrm{~nm}$. Data collected (following DNP) at room temperature and $7 \mathrm{~T}$. (b) Effect of TEMPO concentration on ${ }^{29} \mathrm{Si} \mathrm{T}_{1}$ decay for NPs indicated in (a), measured at room temperature and under $7 \mathrm{~T}$. Inset: ${ }^{29} \mathrm{Si}$ MRI of the Si NP sample (70 nm/30 mM TEMPO) within its sample tube following the corresponding decay study in (b); the MRI was taken 65 min after DNP and after exciting the phantom with a $10^{\circ}$ pulse every $5 \mathrm{~min}$ to acquire the ${ }^{29} \mathrm{Si}$ spectra used for the $T_{1}$ calculation. Photograph to right of ${ }^{29} \mathrm{Si}$ MRI shows sample. (Reprinted with permission from Reference [56]. (C) (2018) American Chemical Society). 


\section{Electron Spin-Dependent Processes in Si Nanocrystals}

\subsection{Excitons in Si Nanocrystals}

The same optical pumping of the electronic system of semiconductor MPs and NPs, which is used for the nuclear spin orientation (see Section 2), can also initiate the electron-spin-dependent processes, which are highly sensitive to both the size of semiconductor particles and their molecular environment. Besides the quantum confinement effect, which results in an increase of the forbidden gap and optical transition energies in nc-Si of different dimensionally and characteristic sizes from 1 to $10 \mathrm{~nm}[57,58]$, the Coulomb interaction between photoexcited electrons and holes in nc-Si promotes the existence of excitons with relatively large binding energies up to $100-200 \mathrm{meV}$ [57] that ensures stability for the excitons at room temperature. Furthermore, the exchange interaction in an exciton can remove the spin degeneracy for the exciton states [59]. It results in two possible spin configurations for the exciton states in nc-Si, which consist of optically active spin-singlet states and optically inactive spin-triplet ones. The triplet exciton state is lowered in energy in respect to the singlet state because of the electron-hole exchange interaction as it was experimentally demonstrated for small nc-Si in electrochemically prepared porous $\mathrm{Si}$ (por-Si) [60]. Despite a small singlet-triplet splitting being in the range from 1 to $30 \mathrm{meV}$ the main part of excitons in nc-Si can be in the threefold degenerated triplet state even at elevated temperatures [61].

The relatively long radiative lifetime of the excitons in nc-Si is due to both a contribution of the indirect optical transitions [61] and the spin-conservation rule [59-61]. In particular, the triplet exciton states are more populated and the corresponding radiative times of the order of several milliseconds and it is detected as the long-lived exciton PL of nc-Si at cryogenic temperatures [60,61]. At room temperature the radiative lifetimes of excitons in nc-Si are partially controlled by the singlet exciton states, which exhibit the radiative lifetimes varied from 10 to $100 \mu \mathrm{s}$, depending on nc-Si dimensions [61]. Because small nc-Si possess a huge accessible internal surface area up to $\sim 1000 \mathrm{~m}^{2} / \mathrm{cm}^{3}$, the electronic surface states and adsorbed molecules can influence the excitons and therefore the photoluminescent properties of nc-Si [62].

\subsection{Photosensitized Generation of Singlet Oxygen}

Since there is a similarity between the electronic structure of excitons in nc-Si and that of many organic molecules as dyes, the energy transfer through spin-split states of the latter can be also probable for the former. A bright example of the spin-dependent energy transfer from photoexcited dye molecules to molecular oxygen in its triplet state followed with its transformation into the singlet oxygen, that is, ${ }^{(1)} \mathrm{O}_{2}$ where (1) in the superscript indicates the electron spin degeneracy, which is used in the photodynamic therapy (PDT) [63]. It was found that nc-Si in por-Si layer and powder can act as a photosensitizer of the ${ }^{(1)} \mathrm{O}_{2}$ generation [64]. The observed photosensitization was explained by the spin-dependent energy transfer from excitons in nc-Si with sizes of 1-10 $\mathrm{nm}$ to $\mathrm{O}_{2}$ molecules adsorbed on the nc-Si surface [65].

According to the quantum mechanics the relaxation of ${ }^{(1)} \mathrm{O}_{2}$ back to the ground (triplet) state is "spin-forbidden" and thus a ${ }^{(1)} \mathrm{O}_{2}$ molecule is characterized by rather long lifetime [66], which is favorable for transferring the excitation energy to oxidation reactions. The latter can be used for the detection and application of ${ }^{(1)} \mathrm{O}_{2}$ [67]. The latter is known to be an electronically excited state of molecular oxygen [67] and it influences oxidative biochemical processes in living systems [68]. The ${ }^{1} \mathrm{O}_{2}$-mediated reaction, which is employed in PDT of cancer, consists of a drug, that is, photosensitizer of ${ }^{(1)} \mathrm{O}_{2}$, administration followed with resonant photoexcitation of the drug [63].

The efficient ${ }^{1} \mathrm{O}_{2}$ photosensitization by nc-Si is promoted by its huge specific surface area up to $10^{3} \mathrm{~m}^{2} / \mathrm{g}$ [62] and by long radiative lifetimes $\left(10^{-5}-10^{-3} \mathrm{~s}\right)$ of the triplet excitons confined in nc-Si [61]. The ${ }^{(1)} \mathrm{O}_{2}$ generation by photoexcited nc-Si can be quantified by measuring the quenching of the exciton photoluminescence (PL) of nc-Si [64] and by analyzing the radiative decay of ${ }^{(1)} \mathrm{O}_{2}$ molecules at $0.98 \mathrm{eV}$ $(1270 \mathrm{~nm})$ as it is usually used for molecular sensitizer [68,69]. In fact, the ${ }^{(1)} \mathrm{O}_{2}$ photosensitization by 
por-Si was successfully monitored by means of the PL spectroscopy of ${ }^{(1)} \mathrm{O}_{2}$ emission at $0.98 \mathrm{eV}$ [70]. While the ${ }^{(1)} \mathrm{O}_{2}$ luminescence indicates a high efficiency of the photosensitization process [71], a strong deactivation (quenching) of ${ }^{(1)} \mathrm{O}_{2}$ molecules in nc-Si assemblies is revealed from a comparison of the photosensitizing efficiency of the standard molecular sensitizer as porphyrin molecules and nc-Si [72]. It can be explained by considering a process of the nonradiative collisional quenching of ${ }^{(1)} \mathrm{O}_{2}$ molecules on the nc-Si surfaces in por-Si layer and powder. The ${ }^{(1)} \mathrm{O}_{2}$ quenching acts as a limiting factor to achieve high concentration of ${ }^{(1)} \mathrm{O}_{2}$ due to the photosensitized generation in por-Si MPs [73].

A method of the electron paramagnetic resonance (EPR) is well known to detect ${ }^{(1)} \mathrm{O}_{2}$ in gaseous ambient [67]. The conventional X-band EPR spectroscopy of photoexcited por-Si in $\mathrm{O}_{2}$ atmosphere allowed us to estimate the ${ }^{(1)} \mathrm{O}_{2}$ concentration photosensitized by nc-Si to be of the order of $10^{19} \mathrm{~cm}^{-3}$ under excitation with photon flux about $10^{20} \mathrm{~cm}^{-2}$ [74]. Similar results were obtained by means of the Q-band EPR spectroscopy, which demonstrated a conversion of about $30 \%$ of $\mathrm{O}_{2}$ molecules in the singlet state upon excitation of por-Si powder in oxygen atmosphere [75]. The ${ }^{(1)} \mathrm{O}_{2}$ photosensitization was shown to be also possible for por-Si dispersed in different oxygen-saturated organic and inorganic liquids [70] as well in water [76].

The quantum theory describes the ground energy state of a $\mathrm{O}_{2}$ molecule as two different molecular orbitals located at two different oxygen atoms with the total spin equals to 1 , which is denoted as ${ }^{3} \Sigma$ state. The electronically excited states are singlet ones and they are characterized by the energy excess of about $0.98 \mathrm{eV}\left({ }^{1} \Delta\right.$ state) and $1.63 \mathrm{eV}\left({ }^{1} \Sigma\right.$ state) in respect to the ground one. The electron transition from the ${ }^{(3)} \mathrm{O}_{2}$ state to ${ }^{(1)} \mathrm{O}_{2}$ one is a spin-flip process and the direct state conversion via absorption of photons is spin-forbidden according to the spin-selection rule. Since the photon absorption and emission are mirror-like processes the pure radiative decay lifetimes of the ${ }^{1} \Delta$ and ${ }^{1} \Sigma$ states are extremely long-about $45 \mathrm{~min}$ and $7 \mathrm{~s}$ respectively [66]. Note, the observed lifetimes of the singlet states are considerably shorter in condensed phases $\left(\sim 10^{-9} \mathrm{~s}\right.$ and $\sim 10^{-3} \mathrm{~s}$ for the ${ }^{1} \Sigma$ and ${ }^{1} \Delta$ states respectively) because of collisions of ${ }^{(1)} \mathrm{O}_{2}$ with other molecules [68].

For the first time, the strong interaction of oxygen molecules with photoexcited nc-Si in por-Si was observed at cryogenic temperatures [64] and it was further studied in more details at different excitation conditions and temperatures including room temperature [65]. The investigated samples of por-Si were prepared by standard electrochemical etching of c-Si wafers in a solution of hydrofluoric acid [77]. In References [64,65] free standing por-Si layers were dried in air for several hours and a part of the prepared por-Si was milled to get powder of MPs assembled from nc-Si and their agglomerates. The samples were kept in a vacuum chamber in oil-free vacuum with a residual pressure of about $10^{-5}$ Torr. The exciton PL spectrum of nc-Si measured in vacuum at low temperature is characterized by a broad, featureless emission band located in the visible region (dashed line in Figure 21). The spectral position and bandwidth reflect the wide band-gap distribution of the nc-Si assembly. Intrinsic defects commonly attributed to Si dangling bonds (DBs) are responsible for the infrared PL band below $1 \mathrm{eV}$ [61]. The adsorption of $\mathrm{O}_{2}$ on nc-Si surface resulted in a strong suppression of both the exciton $\mathrm{PL}$ and the defect PL band [65]. The PL quenching by $\mathrm{O}_{2}$ adsorption is maximal for the PL photon energies above $1.63 \mathrm{eV}$ (Figure 21). The latter value corresponds to the ${ }^{3} \Sigma \rightarrow{ }^{1} \Sigma$ transition energy in $\mathrm{O}_{2}$ molecule. At the same time, the PL line at $0.98 \mathrm{eV}$, which is related to the radiative transition ${ }^{1} \Delta \rightarrow{ }^{1} \Sigma$, appeared after oxygen adsorption in por-Si (inset in Figure 21). These spectroscopic data manifest the (1) $\mathrm{O}_{2}$ photosensitization by the energy transfer from excitons in nc-Si [65]. 


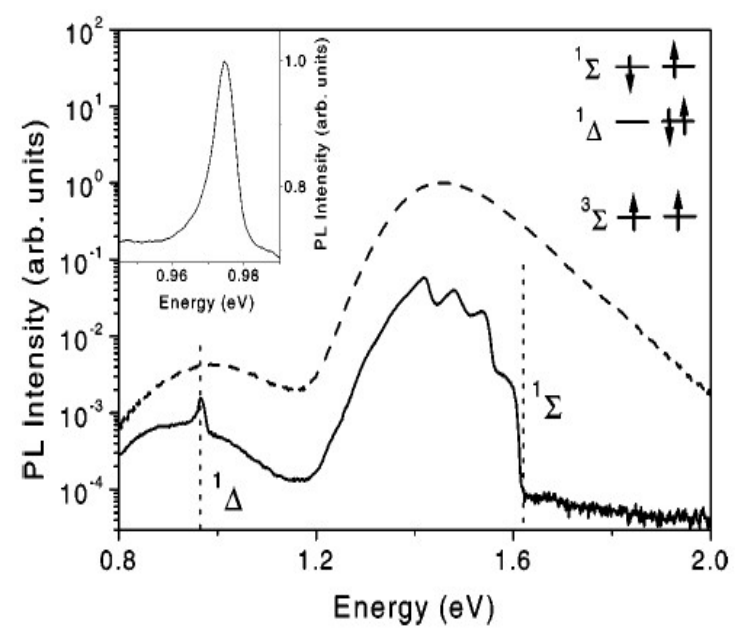

Figure 21. PL spectra of por-Si in vacuum (dashed line) and those with adsorbed oxygen molecules (solid line). Transition energies from the excited states $\left({ }^{1} \Delta,{ }^{1} \Sigma\right)$ of ${ }^{(1)} \mathrm{O}_{2}$ to the ground state are indicated by dashed vertical lines. The spin configuration and spectroscopic labeling of the oxygen states are shown in the right inset. The left inset depicts the PL line related to the ${ }^{1} \Delta \rightarrow{ }^{3} \Sigma$ transition in $\mathrm{O}_{2}$. Temperature $T=5 \mathrm{~K}$, excitation at $2.41 \mathrm{eV}$. (From Reference [65]).

Detailed description of the energy-transfer mechanism responsible for the photosensitized generation of ${ }^{1} \mathrm{O}_{2}$ can be obtained from the spectral dependence of the PL quenching strength, which can be defined as follows [65]:

$$
Q=I_{\mathrm{PL}}{ }^{\mathrm{vac}} / I_{\mathrm{PL}}{ }^{\mathrm{ox}}
$$

where $I_{\mathrm{PL}}{ }^{\mathrm{vac}}$ and $I_{\mathrm{PL}}{ }^{\text {ox }}$ are the exciton PL intensities of nc-Si in vacuum and in oxygen ambient, respectively.

The spectra of $Q\left(h v_{P L}\right)$ and its second derivative are shown in Figure 22. Besides the strongest quenching at $1.63 \mathrm{eV}$ there is an additional maximum at $h v_{P L}$ of about $57 \mathrm{meV}$ below the ${ }^{1} \Sigma$ state energy and numerous maxima separated by energy of about $63 \mathrm{meV}$. These maxima correspond to the energy transfer from nc-Si with band gaps, which do not satisfy to the resonance with the excitation energies of ${ }^{(1)} \mathrm{O}_{2}$. A local maximum separated by $57 \mathrm{meV}$ from the main maximum at $1.63 \mathrm{eV}$ is an evidence of the indirect radiative transition in $\mathrm{nc}-\mathrm{Si}$, where the participation of the momentum conserving $\mathrm{TO}$ phonon with energy of $57 \mathrm{meV}$ is required [61]. The excess of the exciton energy with respect to the energies of the ${ }^{1} \Delta$ and ${ }^{1} \Sigma$ states is compensated by emission of the TO phonons with energy of $63 \mathrm{meV}[64,65]$.

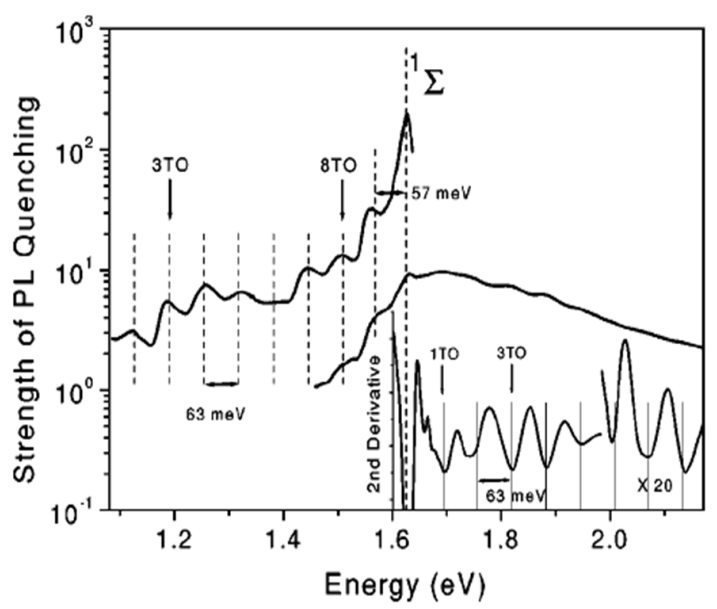

Figure 22. Spectral dependence of the PL quenching strength of por-Si with adsorbed $\mathrm{O}_{2}$ molecules at $T=5 \mathrm{~K}$. Inset shows the second derivative of the PL quenching strength. For convenient presentation, the data are partially scaled by the indicated multiplication factor. Vertical dotted lines are guide for the eye. (From Reference [65]). 
The dynamics of the energy transfer is deduced from the PL transients of por-Si in the presence of adsorbed $\mathrm{O}_{2}$ molecules [65]. The PL spectra of por-Si at different quenching level by the energy transfer to $\mathrm{O}_{2}$ at low temperatures and the corresponding PL decay times $\tau_{\mathrm{PL}}$ are shown in Figure 23 . In vacuum $\tau_{\mathrm{PL}}$ is approximately equal to the radiative lifetime of excitons $\tau_{\mathrm{ex}}$ of the order of $10^{-3} \mathrm{~s}$ and it does not depend on the photon energy (see Figure 23). The PL quenching by $\mathrm{O}_{2}$ adsorption is accompanied by a significant decrease of the PL decay time because the adsorbed $\mathrm{O}_{2}$ molecules on the nc-Si surface represent a channel for the nonradiative recombination of the excitons. The spectral dependence of $\tau_{\mathrm{PL}}$ follows the quenching strength spectrum. The shortest $\tau_{\mathrm{PL}}$ is observed at the PL photon energies, which correspond the strongest PL quenching. Increasing the concentration of adsorbed $\mathrm{O}_{2}$ molecules results in a stronger PL quenching and further decrease of $\tau_{\mathrm{PL}}$ [65]. The time resolved PL studies performed with nanosecond time resolution confirm this spectral behavior of the exciton lifetime [71].

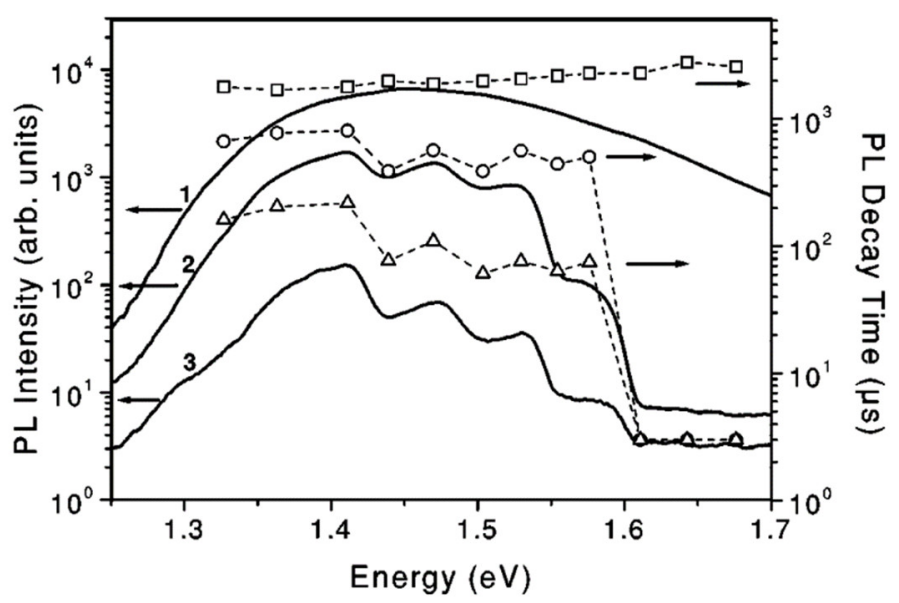

Figure 23. PL spectra (curves) of por-Si and the corresponding spectral dependences of the PL decay time (symbols) for different strength of the quenching induced by $\mathrm{O}_{2}$ molecule adsorption at $T=5 \mathrm{~K}$ : in vacuum (curve 1, squares), intermediate level of the quenching (curve 2, circles) and strong quenching (curve 3, triangles). (From Reference [65]).

The average time of the energy transfer from excitons in nc-Si assembly to $\mathrm{O}_{2}$ molecules can be calculated from the following expression [65]:

$$
\tau_{\mathrm{ET}}{ }^{-1}=\tau_{\mathrm{PL}}{ }^{-1}-\tau_{\mathrm{ex}}{ }^{-1}
$$

In the regime of the strongest quenching the values of $\tau_{\mathrm{ET}}$ for the ${ }^{1} \Delta$ and ${ }^{1} \Sigma$ states are 50 to 100 $\mu \mathrm{s}$ and $<3 \mu \mathrm{s}$, respectively. A uniform concentration of $\mathrm{O}_{2}$ molecules for all nc-Si would result in a quenching strength that scales as $\tau_{\mathrm{ex}} / \tau_{\mathrm{PL}}$. Obviously this simple relation is not valid. A decay time longer than that expected from the quenching strength is observed because of statistical fluctuations of the number of adsorbed $\mathrm{O}_{2}$ molecules - while mainly the slowest PL emission from nc-Si with a small number of the adsorbed molecules contributes to the PL decay, the PL intensity spectrum reflects the response of all nanocrystals [65].

A mechanism of the direct electron exchange between nc-Si and $\mathrm{O}_{2}$ molecule is explained the triplet-triplet annihilation of excitons accompanied by spin-flip excitation of an $\mathrm{O}_{2}$ molecule and followed by the ${ }^{(1)} \mathrm{O}_{2}$ formation [65]. The energy transfer rate is supposed to be determined by the spatial overlap of the electronic wave functions of the interacting species and depends exponentially on the donor-acceptor distance [78]. The latter parameter controls the energy transfer probability, which is larger for nc-Si in as-prepared por-Si with hydrogen-terminated surface [65]. For oxidized nc-Si the increased spacing between confined excitons and adsorbed $\mathrm{O}_{2}$ molecules is of the order of double the length of the Si-O bond $(\sim 3 \AA)$. This critically affects the efficiency of the electron exchange 
interaction. Contrary to a strong coupling for hydrogen-terminated nanocrystals, the PL quenching efficiency is reduced by orders of magnitude if a thin oxide barrier is present. The degree of spatial overlap of the electronic wave functions of confined excitons and adsorbed oxygen molecules depends on the size of nc-Si. For smaller nc-Si the overlap becomes better and the probability for energy transfer is higher. On the other hand, for smaller NPs a larger number of emitted phonons is required to conserve the energy, which lowers the probability for energy exchange. The interplay between these two tendencies results in an almost spectrally constant efficiency of the energy transfer. While the involved transitions are spin forbidden in isolated nc-Si and $\mathrm{O}_{2}$ molecules, they become allowed through exchange interaction [65].

To demonstrate the role of the electron spin statistics for the energy transfer rate, the magnetic-field dependence of the PL quenching efficiency was studied [65]. A magnetic field introduces the quantization axis for electron spins and it results in the lifting of the energy state degeneracy in both $\mathrm{O}_{2}$ molecules and nc-Si. In general, the number of possible states participating in the electron exchange is reduced and the decreased energy transfer rate results in a weaker PL quenching. At low temperature the magnetic field results in preferential occupation of "spin-down" states for both $\mathrm{O}_{2}$ and excitons, while to proceed with energy exchange "spin-up states" are required. The PL quenching was observed at temperature of $10 \mathrm{~K}$ in magnetic fields up to $10 \mathrm{~T}$ as shown in Figure 24. For the spectral region above the ${ }^{1} \Sigma$ state, the high quenching level of the PL intensity prevented the observation of magnetic field effects within the experimental detection sensitivity.

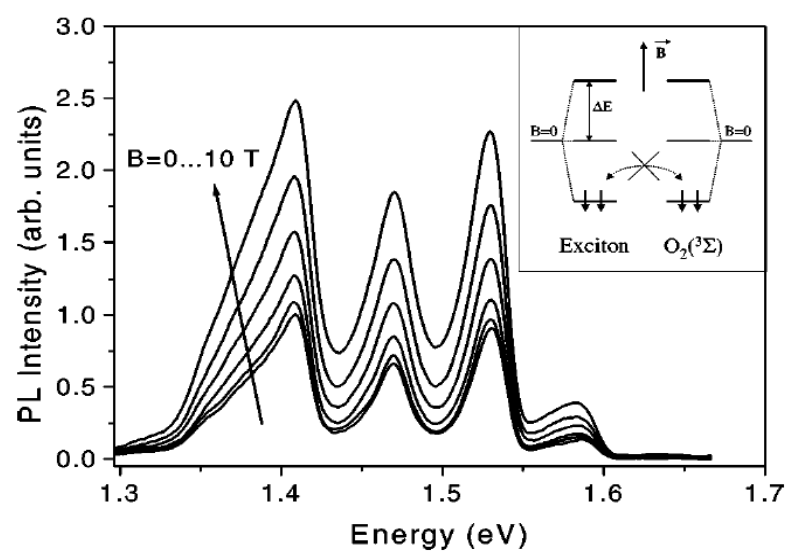

Figure 24. PL spectra of por-Si with adsorbed $\mathrm{O}_{2}$ molecules at various strength of magnetic field at $T=10 \mathrm{~K}$. The magnetic-field increment is equal to $2 \mathrm{~T}$. Inset: Sketch of the Zeeman splitting of the triplet states of excitons in nc-Si and the ground state ${ }^{3} \Sigma$ of $\mathrm{O}_{2}$. The spin orientation for electrons in the lowest-lying levels are indicated by arrows. (From Reference [65]).

Besides the quenching of exciton PL, the photosensitized generation of ${ }^{(1)} \mathrm{O}_{2}$ can be observed by measuring the infrared $\mathrm{PL}$ related to the ${ }^{(1)} \mathrm{O}_{2}$ radiative de-excitation $[64,65]$. The relevant time scale of the infrared PL was determined from time-resolved measurements. In the presence of adsorbed $\mathrm{O}_{2}$ molecules the radiative decay of the ${ }^{1} \Delta$ state of ${ }^{(1)} \mathrm{O}_{2}$ was observed at detection energy of $0.953 \mathrm{eV}$. The initial fast decay is attributed to the defect-related PL background. The slow component with the lifetime $\tau_{\Delta} \approx 0.5 \mathrm{~ms}$ accounts for the ${ }^{1} \Delta \rightarrow{ }^{3} \Sigma$ transition in $\mathrm{O}_{2}$. This lifetime is significantly shorter that the radiative lifetime of a free $\mathrm{O}_{2}$ molecule. The shortening of $\tau_{\Delta}$ can be explained by the interaction of the ${ }^{1} \Delta$ state of ${ }^{(1)} \mathrm{O}_{2}$ with nc-Si surface. The same interaction should also influence the radiative lifetime $\tau_{\mathrm{r}}$ of adsorbed ${ }^{(1)} \mathrm{O}_{2}$ molecules. Firstly, the quantum yield $\eta_{r} \approx 7 \cdot 10^{-5}$ of the radiative decay of the ${ }^{1} \Delta$ state is estimated from the comparison from the integral intensity of the exciton PL band. The radiative lifetime of ${ }^{(1)} \mathrm{O}_{2} \tau_{r} \approx 7 \mathrm{~s}$ is obtained by using the simple relation $\tau_{r}=\tau_{\Delta} / \eta_{r}$. On the one hand, the obtained $\tau_{r}$ is much shorter than the intrinsic radiative lifetime $\sim 50 \mathrm{~min}$. On the other hand, $\tau_{r} \approx 7 \mathrm{~s}$ is close to the ${ }^{1} \Delta$ radiative lifetimes of ${ }^{(1)} \mathrm{O}_{2}$ in different liquids. Therefore, the photoexcited $\mathrm{Si}$ 
nanocrystals contribute simultaneously to the photosensitization process and to the quenching of ${ }^{(1)} \mathrm{O}_{2}$ in por-Si layers and MPs.

\subsection{Phenomenological Model of the Photosensitization by Si Nanocrystals}

The photosensitized generation of ${ }^{(1)} \mathrm{O}_{2}$ in nc-Si ensembles is quantitatively explained by using a model based on the rate equations for excitons and oxygen molecules [65,79]. In brief, the number of excitons in the nc-Si ensemble at low temperatures is limited due to the long radiative lifetime of excitons and efficient nonradiative Auger recombination in nc-Si [80]. According to the experimental data the PL intensity of por-Si in vacuum increases linearly with the excitation intensity up to $0.01 \mathrm{~W} / \mathrm{cm}^{2}$ as shown in Figure 25 [65]. At higher excitation intensities an occupation of each nc-Si by two electron-hole pairs can be achieved and the nonradiative Auger recombination results in a saturation behavior of the emission intensity (triangles in Figure 25). The model describes the experimental data both for por-Si in vacuum (curve 1 ) and after oxygen adsorption, which leads to the superlinear dependence $I_{\mathrm{PL}}$ on $I_{\text {ex }}$ (curves 2,3). The fitting parameters correspond to the product of $\left(\beta \cdot n_{0}\right)^{-1}=200 \mu \mathrm{s}$, which is close to the experimentally determined $\tau_{\mathrm{ET}}$ (see Figure 25 ). The fitting parameter $\beta$ can be calculated by considering the microscopic mechanism of the energy transfer [78].

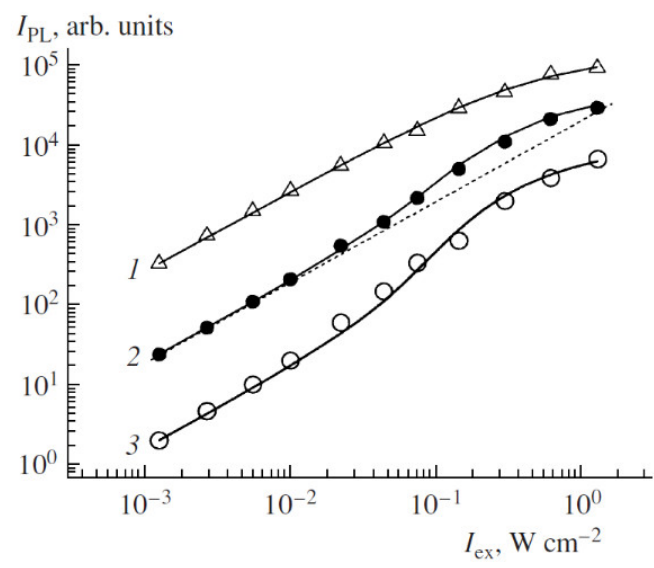

Figure 25. PL intensity of por-Si in vacuum (triangles, $h v_{\mathrm{PL}}=1.47$ and $1.5 \mathrm{eV}$ ) and with adsorbed $\mathrm{O}_{2}$ molecules (closed circles, $h v_{\mathrm{PL}}=1.47 \mathrm{eV}$ and open circles, $h v_{\mathrm{PL}}=1.5 \mathrm{eV}$ ) vs excitation intensity. Dashed line is a linear law approximation. The datasets are arbitrarily scaled for clarity. Solid lines are the fits by using Equations (16) and (17) with the following parameters: $N=10^{19} \mathrm{~cm}^{-3}, \sigma=10^{-15} \mathrm{~cm}^{2}$, $\tau_{\mathrm{ex}}=0.8 \mathrm{~ms}, \tau_{\Delta}=4 \mathrm{~ms}, \beta=5 \cdot 10^{-16} \mathrm{~cm}^{3} / \mathrm{s}$ and $n=0$ (curve 1 ) and oxygen concentrations are taken as $n=10^{19} \mathrm{sm}^{-3}$ (curve 2), $n=3 \cdot 10^{19} \mathrm{~cm}^{-3}$ (curve 3). Excitation photon energy $E_{\text {exc }}=2.41 \mathrm{eV}, \mathrm{T}=5 \mathrm{~K}$. (The experimental data are taken from Reference [65]. Reprinted from Reference [79] by permission from Springer Nature Customer Service Centre GmbH. Springer (C) 2007).

A coupled system, which consists of nc-Si and $\mathrm{O}_{2}$ molecules, under optical excitation can be described by a simple set of the rate equations [79]:

$$
\frac{d N_{1}}{d t}=N_{0} \sigma \Phi-\frac{N_{1}}{\tau_{e x}}-\beta N_{1} n_{0}, \frac{d n_{1}}{d t}=\beta N_{1} n_{0}-\frac{n_{1}}{\tau_{\Delta}},
$$

where $N_{0}$ and $N_{1}$ are the numbers of nc-Si containing 0 and 1 exciton, respectively, $n_{0}$ and $n_{1}$ are the numbers of $\mathrm{O}_{2}$ molecules being in the ground ${ }^{3} \Sigma$ and singlet ${ }^{1} \Delta$ states, respectively, $\tau_{\text {ex }}$ is the exciton lifetime, $\tau_{\Delta}$ is the lifetime of the ${ }^{1} \Delta$ state, $\sigma$ is the cross section of optical absorption of nc-Si, $\Phi$ is the photon flux density of exciting light, $\beta$ is the coupling coefficient. The latter value describes the energy transfer probability of in nc-Si/O $\mathrm{O}_{2}$ system and the reciprocal product $\left(\beta n_{0}\right)^{-1}$ corresponds to the experimentally accessible energy-transfer time $\tau_{\mathrm{ET}}$. 
To solve Equation (16) the following expressions for the total numbers of interacting species can be used:

$$
N=N_{0}+N_{1}, n=n_{0}+n_{1},
$$

where $N$ and $n$ are the total numbers of nc-Si and $\mathrm{O}_{2}$ molecules, respectively. The discussed phenomenological model does not account contributions of the upper excited states of nc-Si and $\mathrm{O}_{2}$ molecules, the nonradiative recombination of excitons in nc-Si and the reverse energy transfer from ${ }^{(1)} \mathrm{O}_{2}$ to nc-Si.

The exciton PL intensity of nc-Si is obtained from the steady state solution of Equations (16) and (17) and from the relation $I_{\mathrm{PL}}=N_{1} / \tau_{\mathrm{ex}}$. The time constants used in the model are obtained from the experimental data discussed above whereas $\sigma$ is known from Reference [61]. Solutions of Equation (16) fit the experimental dependences of the PL intensity of por-Si on excitation intensity $I_{\mathrm{ex}}$ (solid lines in Figure 25).

The described model assumes that the larger number of adsorbed $\mathrm{O}_{2}$ molecules results in shorter $\tau_{\mathrm{ET}}$ at the same conditions of the energy transfer. The energy transfer efficiency is described by $\beta$ and it is obviously dependent on nc-Si size and surface conditions [79]. Hydrogen terminated nc-Si assembles with lowest number of nonradiative surface defects as $\mathrm{Si} D B s$ are preferable for the ${ }^{(1)} \mathrm{O}_{2}$ photosensitization [81]. For por-Si aged in air at room temperature, the photosensitization process is additionally complicated by the thermally activated desorption of $\mathrm{O}_{2}$ molecules as well as by the thermally activated exciton migration and nonradiative recombination. Besides, it should be analyzed both the generation rate and the quenching process of ${ }^{(1)} \mathrm{O}_{2}$ via the interaction with internal surface of por-Si and ambient molecules. The quenching processes are especially important for por-Si suspended in different solvents. The theoretical description of the photosensitized generation of ${ }^{(1)} \mathrm{O}_{2}$ by real systems based on por-Si needs rather sophisticate analysis. The experimental results presented in the next sections of this chapter can be used as experimental basis for future complete theory of the photosensitized generation of ${ }^{(1)} \mathrm{O}_{2}$ by nc-Si.

Contrary to cryogenic temperatures, the conditions of efficient interaction between excitons in nc-Si and $\mathrm{O}_{2}$ molecules adsorbed on the surfaces of nc-Si are not fulfilled at elevated temperatures. First of all, because of the thermally activated desorption, a small spatial separation between excitons and $\mathrm{O}_{2}$ molecules is realized only during a short time of collisions [65]. Furthermore, the exciton lifetime and spin-triplet state occupation drop down with rising temperature [59-61]. It results in a weaker PL quenching, which scales with the collision rate, that is, the gas pressure and in a broad spectral resonance of the energy transfer to the ${ }^{1} \Sigma$ state [64]. Spectra of the photosensitization efficiency of nc-Si in por-Si at different temperatures are shown in Figure 26 [65]. At intermediate temperatures $(T=110 \mathrm{~K})$ the second quenching band in the spectral region of $1.75-1.95 \mathrm{eV}$ is observed and it becomes better pronounced with increasing oxygen concentration (Figure 26a). This quenching band is attributed to the energy transfer from excitons confined in nc-Si to ${ }^{(1)} \mathrm{O}_{2}$ dimers, that is, to the $2\left({ }^{1} \Delta\right)$ state formation [65]. The energy transfer to the $2\left({ }^{1} \Delta\right)$ states is enhanced at higher pressures due to increasing the probability of dimer formation. A decrease of the dimer-related quenching band is observed at higher temperature because the dimer formation is less probable. For temperatures above $250 \mathrm{~K}$ the energy transfer to the ${ }^{(1)} \mathrm{O}_{2}$ dimers cannot be resolved spectroscopically (see Figure $26 \mathrm{~b}$ ). This fact agrees with results of the spectroscopic investigation of ${ }^{(1)} \mathrm{O}_{2}$ [67]. 


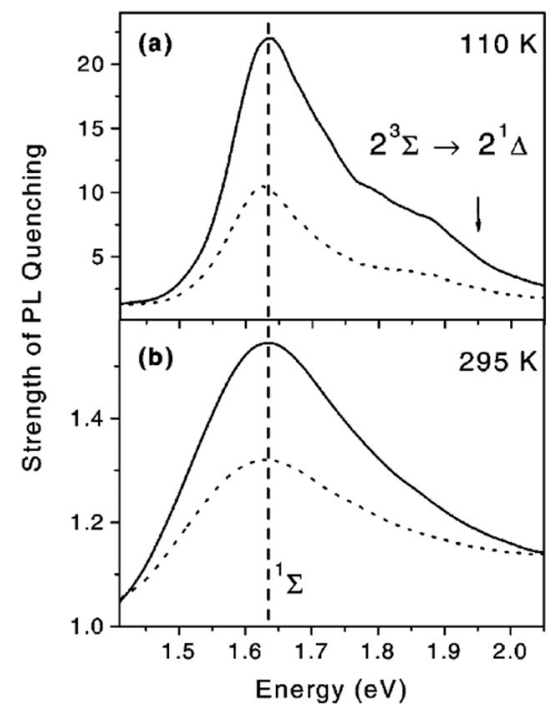

Figure 26. (a) Spectra of the PL quenching strength in por-Si with adsorbed $\mathrm{O}_{2}$ molecules at $\mathrm{T}=110 \mathrm{~K}$, 100 mbar (solid line) and 2 mbar (dotted line). (b) the same spectra at T=295 K, 1 bar (solid line) and 100 mbar (dotted line). (From Reference [65]).

Dependence of the exciton PL quenching detected at $1.63 \mathrm{eV}$ on the $\mathrm{O}_{2}$ ambient pressure at different temperatures are shown in Figure 27 [65]. The pressure dependence is well described by using the Langmuir approach for molecular adsorption on a surface [82], that considers the dynamic equilibrium between the rates of adsorption and desorption by the following Equation:

$$
Q^{-1}=1-A \frac{K P_{o x}}{1+K P_{o x}}
$$

where $K$ is the equilibrium constant, $P_{o x}$ is the oxygen gas pressure and $A$ is the part of exciton energy transferred to adsorbed $\mathrm{O}_{2}$ molecules [65].

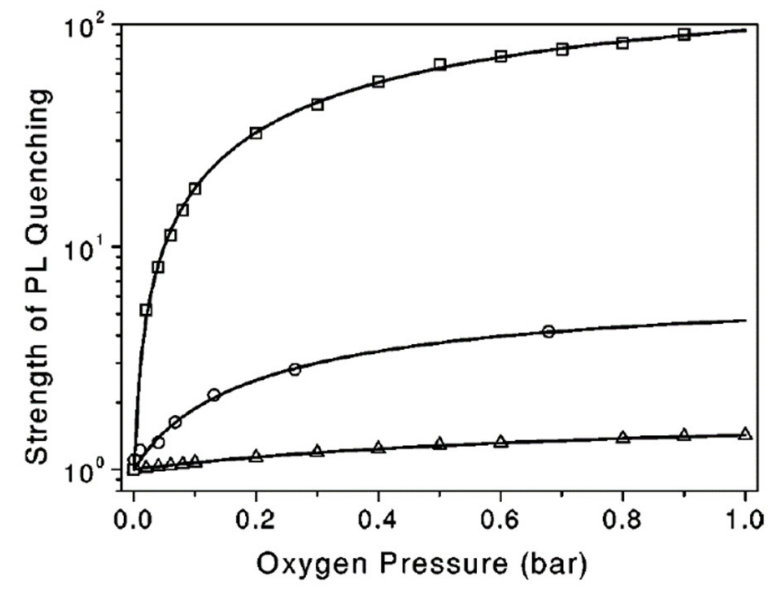

Figure 27. Dependences of the exciton PL quenching strength at $1.63 \mathrm{eV}$ on oxygen pressure at different temperatures: $T=120 \mathrm{~K}$ (squares), $T=200 \mathrm{~K}$ (circles), $T=300 \mathrm{~K}$ (triangles). Solid lines are fits by Equation (18). (From Reference [65]).

The experimental pressure dependence of the photosensitization efficiency is well described by theoretical predictions (solid lines in Figure 27). As expected, in the entire pressure range a decrease of the temperature increases the mean number of adsorbed molecules and enhances the efficiency of energy transfer to $\mathrm{O}_{2}$ molecules. The steady state concentration of ${ }^{(1)} \mathrm{O}_{2}$ is a product of its generation 
rate and the collision-related deactivation one. Because both quantities are rising with oxygen ambient pressure [67], there is an optimal $P_{o x}$ for the maximal steady state concentration of ${ }^{(1)} \mathrm{O}_{2}$ [65].

Additional information about the ${ }^{(1)} \mathrm{O}_{2}$ photosensitization at room temperature is obtained from the PL transient measurements. Figure 28 presents several transients of the exciton PL of por-Si MPs in gaseous oxygen ambient. The inset of Figure 28 shows the transients after the pumping of oxygen molecules. The PL transients are not actually single exponential. However, they can be well fitted by single exponential decays for times about $50-70 \mu$ s after the excitation laser pulse [83]:

$$
I_{P L}(t)=I_{P L}(0) \exp \left(-t / \tau_{P L}\right),
$$

where $\tau_{P L}$ is the PL lifetime and $I_{P L}(0)$ is the PL intensity just after excitation. The values of $\tau_{P L}$ for the samples in vacuum are found to be within a microsecond time scale that is in good agreement with the singlet lifetimes of excitons in nc-Si [60,61].

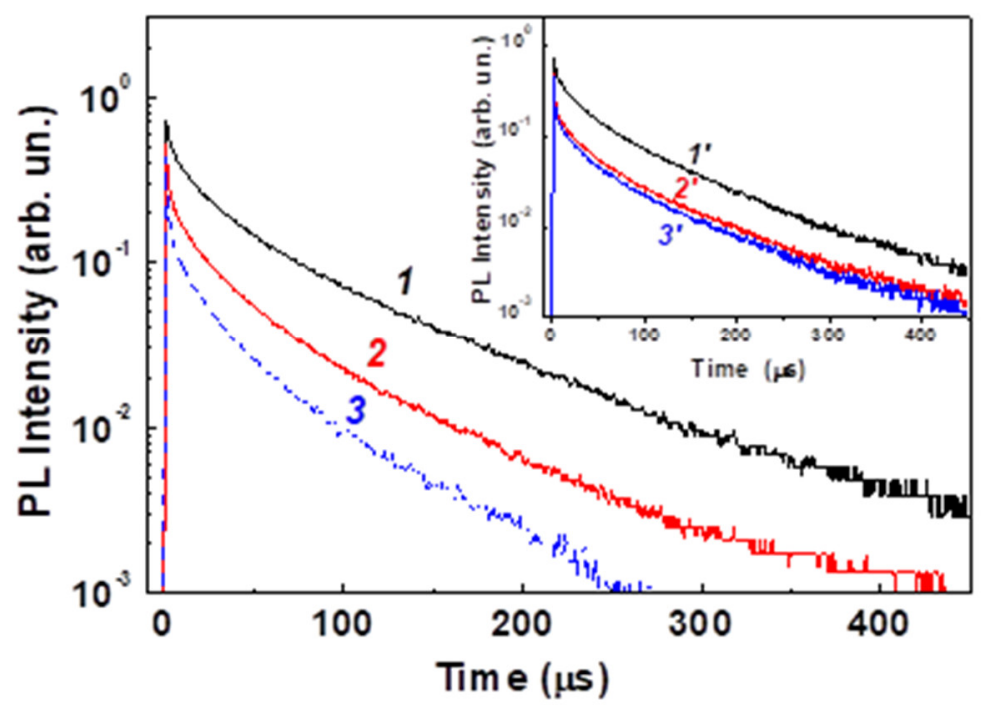

Figure 28. Transients of the exciton PL intensity of nc-Si in por-Si at $1.6 \mathrm{eV}$ at oxygen pressures $P_{\text {ox }}=1$ Torr (1), 100 Torr (2) and 200 Torr (3). Inset: the same PL transients in vacuum after oxygen adsorption at $P_{\text {ox }}=1$ Torr $\left(1^{\prime}\right), 100$ Torr $\left(2^{\prime}\right)$ and 200 Torr $\left(3^{\prime}\right) . T=300 \mathrm{~K}$. (Reprinted from Reference [83] by permission from John Wiley and Sons (C) 2007).

The admission of $\mathrm{O}_{2}$ causes a decrease of $\tau_{P L}$ and this effect is stronger at higher oxygen pressure (curve 3 in Figure 28). The PL lifetime shortening after oxygen adsorption with $P_{\mathrm{ox}}>10$ Torr is partially reversible (inset of Figure 28). The strongest lifetime shortening occurs at the PL energy of $1.6 \mathrm{eV}$, which correlates with the spectral maximum of the PL intensity quenching.

While the energy transfer time at room temperature can be obtained from the PL decay times by applying Equation (13), in which $\tau_{e x}$ has to be substituted by $\tau_{P L}$ in vacuum, the energy transfer efficiency is estimated by using the following expression [83]:

$$
\eta=1-\int_{0}^{\infty} I_{P L}^{o x}(t) d t / \int_{0}^{\infty} I_{P L}^{v a c}(t) d t
$$

It is obvious that $\eta \approx 1-Q^{-1}$ where $Q$ is described by Equation (14). If the PL quenching is not completely reversible due to the oxidation of nc-Si by photosensitized ${ }^{(1)} \mathrm{O}_{2}$ [81], Equations (15) and (20) will give the lower and upper limits of the corresponding energy transfer time and photosensitization efficiency, respectively [83].

It was found that the photosensitization efficiency in por-Si layers and powder is strongly dependent on the porosity of nc-Si assemblies (Figure 29). The minimal value of $\tau_{\mathrm{ET}}$ and maximal 
value of $\eta$ are supposed for highly porous layers and powder of MPs, which consist of smallest nc-Si. The porosity dependence of $\eta$ and $\tau_{\mathrm{ET}}$ can be explained by considering the following reasons. The first one is a growth of the specific surface area of nc-Si with porosity increasing that promotes the $\mathrm{O}_{2}$ adsorption and then the total rate of ${ }^{(1)} \mathrm{O}_{2}$ photosensitization. The second reason is related to a decrease of the nonradiative recombination with increasing the porosity that results in an increase of the exciton concentration. In fact, the PL quantum yield increases in por-Si with high porosity that indicates the second reason impact [83].

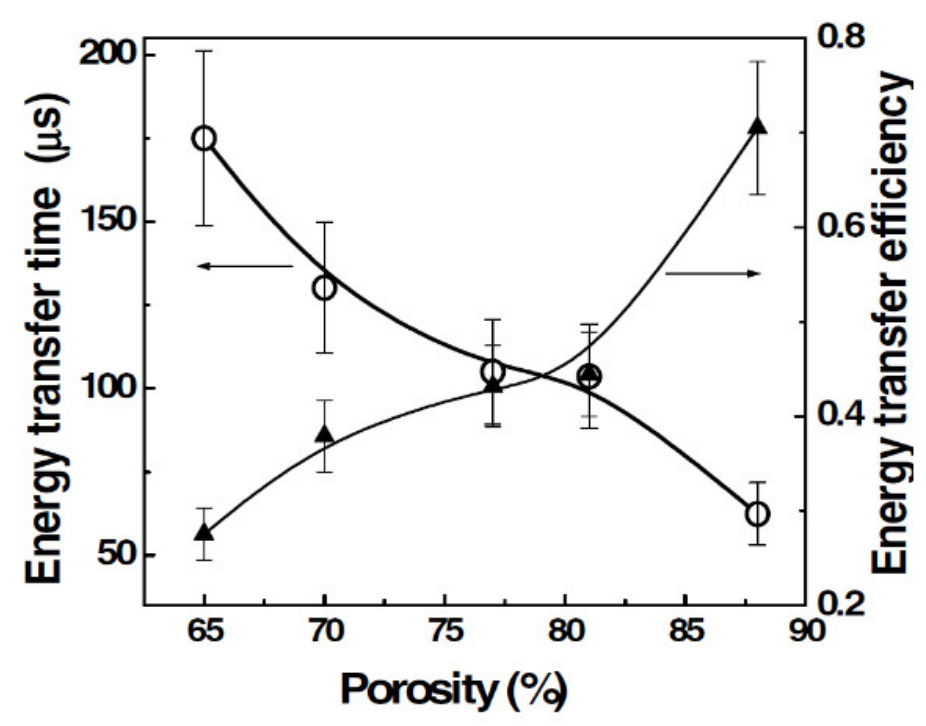

Figure 29. Energy transfer time (circles) and energy transfer efficiency (triangles) vs porosity of por-Si. $E_{\mathrm{exc}}=3.7 \mathrm{eV}, h v_{\mathrm{PL}}=1.6 \mathrm{eV}, P_{\mathrm{ox}}=1 \mathrm{bar}, \mathrm{T}=300 \mathrm{~K}$. (Reprinted from Reference [83] by permission from John Wiley and Sons (C) 2007).

Besides the characteristic energy of about $1.63 \mathrm{eV}$ (the ${ }^{1} \Sigma \rightarrow{ }^{3} \Sigma$ transition energy in $\mathrm{O}_{2}$ molecule), the spectrally selective quenching of the exciton PL intensity in por-Si at room temperature demonstrate an additional maximum at photon energy of $2-2.2 \mathrm{eV}$, which is related to the formation of superoxide radicals, that is, $\mathrm{O}^{-}{ }_{2}$ [76]. The $\mathrm{PL}$ quenching at $2-2.2 \mathrm{eV}$ can be also explained by the charging of nc-Si due to the $\mathrm{O}^{-}{ }_{2}$ radical formation on the nc-Si surface [83] because the positively charged $\mathrm{Si}$ nanocrystals exhibit an enhanced rate of the nonradiative Auger recombination $[61,80]$.

The infrared PL of nc-Si in por-Si MPs at room temperature was investigated to get information on the photosensitized generation of ${ }^{(1)} \mathrm{O}_{2}$ [70]. Since the radiative transitions in ${ }^{(1)} \mathrm{O}_{2}$ molecules are characterized by extremely low probability, the experimental observation of the ${ }^{(1)} \mathrm{O}_{2}$ phosphorescence at $0.98 \mathrm{eV}$ required very sensitive detection system and was possible only for por-Si with rather efficient generation of ${ }^{(1)} \mathrm{O}_{2}$. Figure 30 shows typical infrared PL spectra of por-Si powder obtained by mechanical milling of por-Si films [73]. While the PL spectrum of por-Si powder in vacuum shows a featureless emission band related to defects states in nc-Si [61], the PL line of the ${ }^{(1)} \mathrm{O}_{2}$ phosphorescence at $0.98 \mathrm{eV}$ appears just after the admission of $\mathrm{O}_{2}$ to por-Si powder. For several minutes after the $\mathrm{O}_{2}$ admission the ${ }^{(1)} \mathrm{O}_{2}$ phosphorescence intensity decreases, while the intensity of the defect-related band increases. The relative intensity of the ${ }^{(1)} \mathrm{O}_{2}$ phosphorescence intensity was found to decrease in por-Si subjected to mechanical grinding, which resulted in increasing the defect-related PL [73]. 


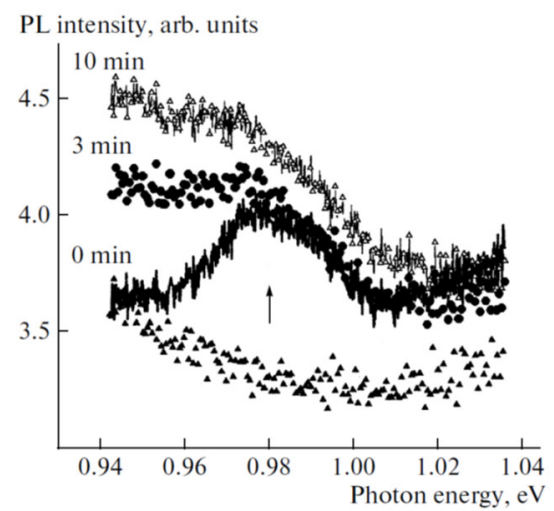

Figure 30. Spectra of the photoluminescence of por-Si powder in vacuum (lowest curve) and in oxygen atmosphere at $P_{\mathrm{ox}}=1 \mathrm{bar}$ for different times after illumination (indicated near the corresponding curves). $E_{\mathrm{exc}}=2.33 \mathrm{eV}, I_{\mathrm{exc}}=0.1 \mathrm{~W} / \mathrm{cm}^{2}, T=300 \mathrm{~K}$. (Reprinted from Reference [73] by permission from Springer Nature Customer Service Centre GmbH: Springer (C) 2010).

Dependences of the intensity of ${ }^{(1)} \mathrm{O}_{2}$ luminescence at $0.98 \mathrm{eV}$ and defect-related PL at $0.94-0.95 \mathrm{eV}$ for por-Si photoexcited at room temperature indicate formation of new defect states on the nc-Si surfaces due to the photosensitized generation of ${ }^{(1)} \mathrm{O}_{2}$ [73]. Most probable reason of the defect formation is the photo-induced oxidation of nc-Si by ${ }^{(1)} \mathrm{O}_{2}$, which is accompanied by irreversible quenching of the exciton PL [81]. The latter process induces decreasing the ${ }^{(1)} \mathrm{O}_{2}$ photosensitization efficiency and then the ${ }^{(1)} \mathrm{O}_{2}$ luminescence intensity drops down (see Figure 31 ). Therefore por-Si acts both as the photosensitizer and quencher of ${ }^{(1)} \mathrm{O}_{2}$. The lifetime $\tau_{\Delta} \approx 15 \mathrm{~ms}$ was estimated from the comparison of ${ }^{(1)} \mathrm{O}_{2}$ luminescence and exciton PL of nc-Si at room temperature at $P_{\mathrm{ox}}=1 \mathrm{bar}$ [73]. On the one hand, the estimated lifetime is significantly shorter than the radiative lifetime of ${ }^{(1)} \mathrm{O}_{2}$ [67]. On the other hand, $\tau_{\Delta} \approx 15 \mathrm{~ms}$ is larger than the ${ }^{(1)} \mathrm{O}_{2}$ lifetime in water $(3 \mu \mathrm{s})$ or biosystems $(<1 \mu \mathrm{s})$ [69]. This fact implies that nc-Si can be considered as a potential photosensitizer of ${ }^{(1)} \mathrm{O}_{2}$ in liquids, including aqueous media $[70,71]$.

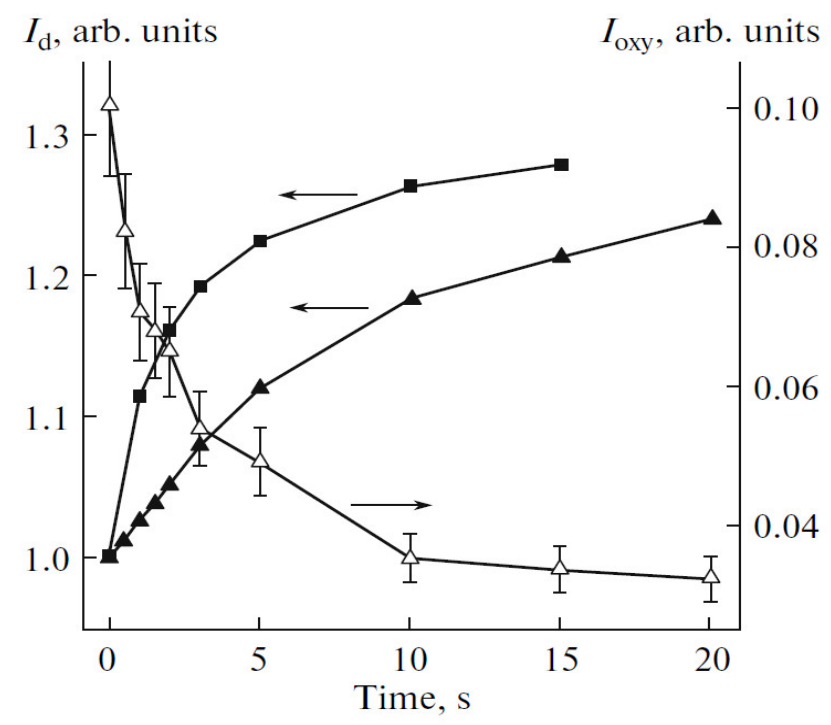

Figure 31. Dependences of the of ${ }^{(1)} \mathrm{O}_{2}$ luminescence intensity, $I_{o x y}$, (open triangles) and defect-related $\mathrm{PL}, I_{d}$, for initial por-Si (solid triangles) and por-Si powder (solid squares) for the photoexcitation time of por-Si powder in oxygen atmosphere at $P_{\mathrm{ox}}=1 \mathrm{bar}$. $E_{\mathrm{exc}}=2.33 \mathrm{eV}, I_{\mathrm{exc}}=0.1 \mathrm{~W} / \mathrm{cm}^{2}, T=300 \mathrm{~K}$. (Reprinted from Reference [73] by permission from Springer Nature Customer Service Centre GmbH: Springer (C) 2010.) 
Beside the PL diagnostics of the nc-Si-induced photosensitization the EPR technique was shown to be efficient for the quantitative analysis of the ${ }^{(1)} \mathrm{O}_{2}$ photosensitization [84]. It is known that por-Si possesses intrinsic defects as $\mathrm{Si}$ DBs, which can act as spin labels for ${ }^{(1)} \mathrm{O}_{2}$. Typical EPR spectra of por-Si are shown in Figure 32. The EPR signal is characterized by the effective $g$-factor of $2.0055 \pm 0.0005$. The corresponding paramagnetic defects are usually attributed to so-called $\mathrm{P}_{\mathrm{b}}$-like centers, which are $\mathrm{Si}$ $\mathrm{DBs}$ at $\mathrm{Si} / \mathrm{SiO}_{2}$ interface [85]. The EPR signal intensity ( $\mathrm{I}_{\mathrm{EPR}}$ ) of por-Si in vacuum is the weakest at high microwave power because of the saturation effect of the microwave power absorbance by Si DBs [74]. The ${ }^{1} \mathrm{O}_{2}$ can be also detected by monitoring a decrease of the concentration of paramagnetic ${ }^{(3)} \mathrm{O}_{2}[75]$.

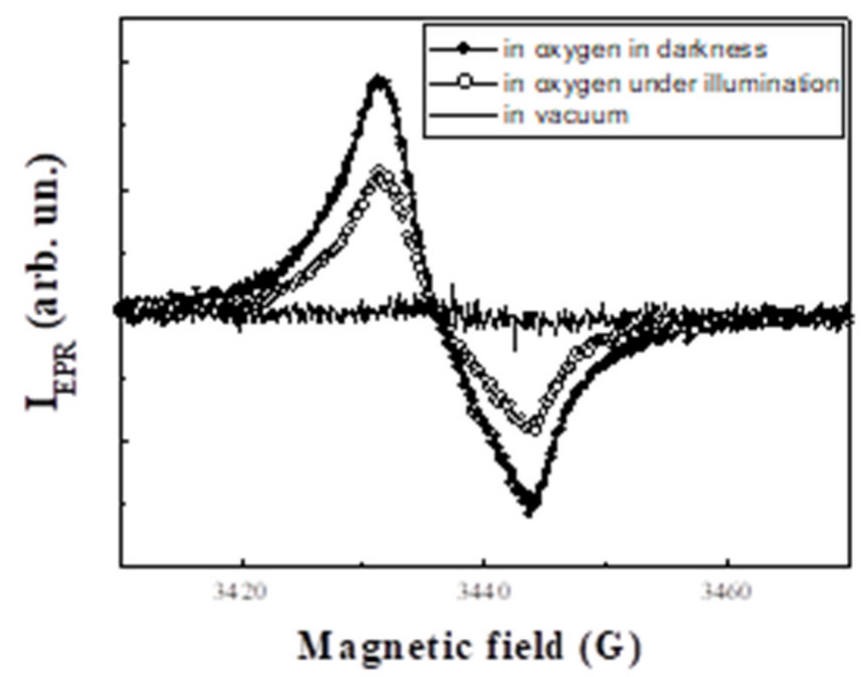

Figure 32. Electron paramagnetic resonance (EPR) spectra of por-Si powder in vacuum and in oxygen ambient $\left(P_{\mathrm{ox}}=760\right.$ Torr $)$ in darkness and under illumination. Measurements were done at high microwave power of about $200 \mathrm{~mW}$. (Reprinted from Reference [84] with permission from Elsevier).

It was found that por-Si in $\mathrm{O}_{2}$ atmosphere was characterized by larger $\mathrm{I}_{\mathrm{EPR}}$ in darkness than under illumination. Furthermore, $\mathrm{I}_{\mathrm{EPR}}$ for the sample under short time illumination in $\mathrm{O}_{2}$ atmosphere decreased almost reversibly [74]. Note, that prolonged intensive illumination led to an irreversible increase of $\mathrm{I}_{\mathrm{EPR}}$ due to the defect formation under photo-oxidation [81]. The saturation effect of the EPR signal of por-Si results from relatively long time of the spin relaxation of $\mathrm{P}_{\mathrm{b}}$-centers [85]. In general, $\mathrm{I}_{\mathrm{EPR}}$ depends on the number of paramagnetic centers (Si DBs in the case of nc-Si) and their relaxation time, which can be expressed in the following way [75]:

$$
\left(T^{D B}\right)^{-1}=\left(2 T_{1}^{D B}\right)^{-1}+\left(T_{2}^{D B}\right)^{-1}
$$

where $T_{1}^{D B}$ is the longitudinal relaxation time (spin-lattice relaxation) and $T_{2}^{D B}$ is the transverse relaxation time, associated with spin-spin relaxation. For nc-Si systems with low defect density $-T_{1}^{D B}$ $<<T_{2}^{D B}$ and then $T \approx 2 T_{1}$. While the value of $T_{1}$ for bulk c-Si at the room temperature lies typically in the range of several microseconds [85], this time can be longer in small nc-Si because of a low density of phonon modes. Indeed, $\mathrm{I}_{\mathrm{EPR}}$ for the sample in vacuum exhibits the strong saturation even at relatively low microwave power (Figure 33). The spectral shape of the EPR signal of the sample in vacuum does not change significantly under illumination [75]. Note, the factor of phonon mode depletion in $\mathrm{nc}-\mathrm{Si}$ can also influence the longitudinal relaxation time of nuclear spin as ${ }^{29} \mathrm{Si}$ what is essential for the hyperpolarization method (see Section 2). 


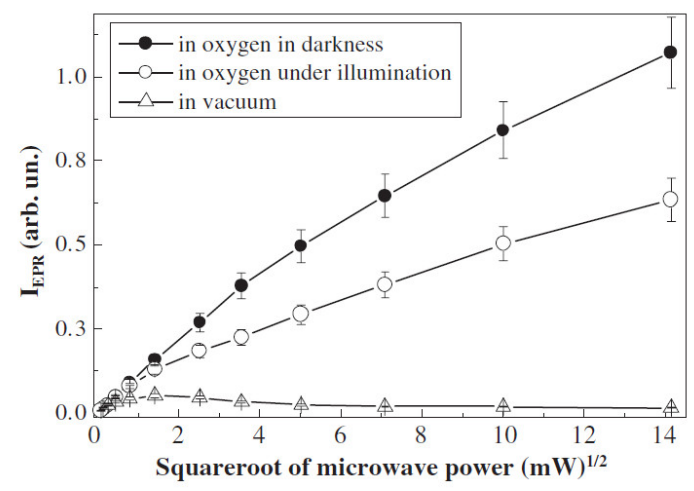

Figure 33. Dependences of the EPR signals of por-Si in vacuum (triangles) and in oxygen ambient $\left(P_{\mathrm{ox}}=760\right.$ Torr) in darkness (solid circles) and under illumination (open circles). (Reprinted from Reference [84] with permission from Elsevier).

The $T_{2}$ value is reciprocal to the paramagnetic center concentration, which depends on the number of adsorbed triplet $\mathrm{O}_{2}$ molecules on nc-Si surface. The saturation of $\mathrm{I}_{\mathrm{EPR}}$ at high microwave power can be absent when $T_{2}$ decreases due to the spin-spin relaxation. Indeed, there is no saturation of the $\mathrm{I}_{\mathrm{EPR}}$ signal on microwave power for por-Si in oxygen atmosphere (Figure 33). Since $\mathrm{I}_{\mathrm{EPR}}$ for the samples at $P_{\text {ox }}=760$ Torr decreases by $40 \%$ under illumination with photon flux of about $10^{20} \mathrm{~cm}^{-2}$, the concentration of triplet $\mathrm{O}_{2}$ molecules decreases by $\Delta n \approx 0.4 n_{0}$, where $n_{0}$ is the initial $\mathrm{O}_{2}$ concentration. The latter number at $P_{\mathrm{ox}}=760$ Torr is about $10^{19} \mathrm{~cm}^{-3}$ (the Avogadro number divided by the molar volume). From the comparison of $I_{\mathrm{EPR}}$ in the $\mathrm{O}_{2}$ atmosphere in darkness and under illumination, one can estimate the ${ }^{(1)} \mathrm{O}_{2}$ molecular concentration to be $\Delta n \approx 4 \cdot 10^{18} \mathrm{~cm}^{-3}$. The latter value is comparable with the number of nc-Si and it is by 2 orders of magnitude larger than the Si DBs concentration. This fact demonstrates that $\mathrm{Si} \mathrm{DBs}$ are only probes to monitor the ${ }^{(1)} \mathrm{O}_{2}$ photosensitization. The observed features of $\mathrm{I}_{\mathrm{EPR}}$ for por-Si in oxygen atmosphere in darkness and under illumination were confirmed by means of the pulsed EPR technique and similar concentration of photosensitized ${ }^{(1)} \mathrm{O}_{2} \sim 10^{18} \mathrm{~cm}^{-3}$ was detected by using the Q-band EPR [75].

While por-Si looks rather efficient photosensitizer of the ${ }^{(1)} \mathrm{O}_{2}$ generation in gaseous atmosphere, the photosensitization by por-Si in liquids and, especially, in aqueous solutions is required for biomedical applications [86-90]. Unfortunately, the fast nonradiative relaxation of ${ }^{(1)} \mathrm{O}_{2}$ in water complicates detection of the ${ }^{(1)} \mathrm{O}_{2}$ luminescence. In this case the ${ }^{(1)} \mathrm{O}_{2}$ photosensitization can be indirectly probed by monitoring the intensity and lifetime of the exciton PL of por-Si. Although the exciton PL quenching for nc-Si dispersed in water is weaker, its spectral shape is similar to that measured in gaseous ambient. Note, the relative contribution of the high-energy quenching at $2-2.2 \mathrm{eV}$ is stronger in water because of more efficient charging of the nc-Si surfaces followed with formation of $\mathrm{O}^{-}{ }_{2}$ radicals [76].

The lifetime of the exciton PL of por-Si MPs dispersed in water decreases after saturation of the solution by oxygen as shown in Figure 34 [91]. The energy transfer times determined from transients of the exciton PL by using Equation (13) are plotted in inset of Figure 34. The spectral dependence of the energy transfer time is similar to that observed for por-Si in gaseous oxygen ambient (see Figure 26). A slightly longer energy transfer time correlates with weaker quenching of the PL intensity of por-Si in water than that in gaseous $\mathrm{O}_{2}$. This fact indicates an influence of water molecules on the exciton recombination and/or energy transfer from the excitons to $\mathrm{O}_{2}$ molecules. Indeed, the energy transfer is mostly efficient for nc-Si with S-H surface bonds, which are hydrophobic [77]. Therefore nc-Si dispersed in water should be partially oxidized that results in decreasing the ${ }^{(1)} \mathrm{O}_{2}$ photosensitization efficiency [65]. The oxidation of nc-Si dispersed in water or aqueous solutions can be a limiting factor to achieve the stable and efficient ${ }^{(1)} \mathrm{O}_{2}$ photosensitization. The oxidation process can be also stimulated during the photosensitization [81]. For biomedical applications of nc-Si in PDT it is obviously necessary to stabilize nc-Si surface against the ${ }^{(1)} \mathrm{O}_{2}$ induced photooxidation. It can be done, for example, by using rapid thermal annealing or laser irradiation. For example, nearly spherical colloidal nc-Si obtained by femtosecond laser ablation of c-Si 
wafer in deuterated water $\left(\mathrm{D}_{2} \mathrm{O}\right)$ water were found to photosensitize the ${ }^{(1)} \mathrm{O}_{2}$ generation with a yield estimated at $10 \%$ of that of the standard molecular sensitizer a photofrin [86].

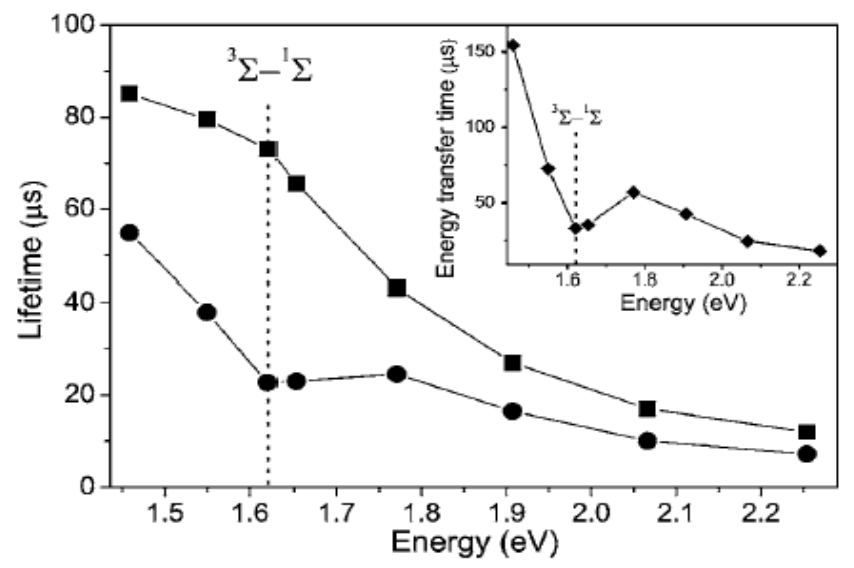

Figure 34. Spectral dependences of the PL lifetime measured for por-Si MPs dispersed in degassed water (squares) and in oxygen-saturated water (circles). Inset: spectral dependence of the energy transfer time in oxygen-saturated water. $E_{\mathrm{exc}}=2.33 \mathrm{eV}, \mathrm{T}=300 \mathrm{~K}$. (From Reference [91]).

\subsection{Si Nanocrystals as Photosensitizers of Singlet Oxygen Generation for Biomedical Applications}

The ${ }^{(1)} \mathrm{O}_{2}$ photosensitization efficiency by nc-Si dispersed in water was confirmed by measurements of the exciton PL of por-Si MPs excited by laser pulses. The relaxation time of the exponential part of the PL transients in the solution without $\mathrm{O}_{2}$ was $\tau_{\mathrm{ex}} \approx 85 \mu \mathrm{s}$, which is close to the natural radiative lifetime of excitons confined in nc-Si. The saturation of the solution with gaseous $\mathrm{O}_{2}$ at a pressure of 760 Torr leads to a decrease in the PL decay time to $\tau_{\mathrm{D}} \approx 50 \mu \mathrm{s}$. The ratio $\tau_{\mathrm{ex}} / \tau_{\mathrm{D}} \approx 1.6$ is close to the measured PL quenching. These observations indicate that the decrease of the PL lifetime of nc-Si in oxygen-saturated suspensions is caused by the photosensitized generation of ${ }^{(1)} \mathrm{O}_{2}$ [87].

The photosensitized generation of ${ }^{\left({ }^{(1)}\right.} \mathrm{O}_{2}$ was confirmed by experiments in vitro, which showed that number of living cells with added por-Si MPs decreased strongly after photoexcitation as compared to the reference group (see Figure 35). At the same time, the effect of por-Si MPs was almost absent over the entire concentration range in darkness. This fact is concerned with the ${ }^{(1)} \mathrm{O}_{2}$ generation process under the illumination in the active solution with dispersed nc-Si, because ${ }^{(1)} \mathrm{O}_{2}$ being strong oxidizing substance results in both killing of cells and the inhibitions of cell proliferation [87]. An analysis of the DNA content of the cells incubated with photoexcited por-Si MPs indicates smaller DNA content is typical for the cell death due to the apoptosis mechanism [88].

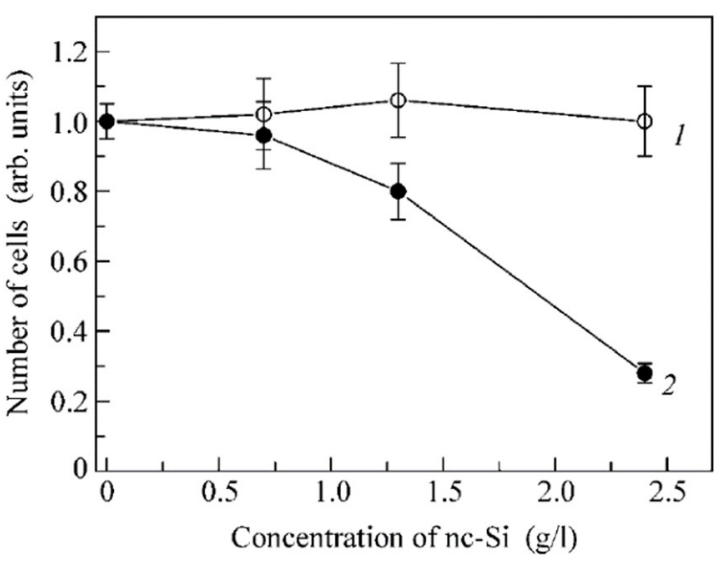

Figure 35. Concentration dependence of the relative number of mouse fibroblast cells with added por-Si MPs in darkness (curve 1) and after illumination with white light source for $1 \mathrm{~h}$ (curve 2). (Reprinted from Reference [87] by permission from Springer Nature Customer Service Centre GmbH: Springer (C 2006). 
Mesoporous Si NPs with mean sizes of the order of $100 \mathrm{~nm}$ and pore size of $12 \mathrm{~nm}$ were used to enhance the photosensitized generation of ${ }^{(1)} \mathrm{O}_{2}$ with quantum yield $\sim 10 \%$ in ethanol and $17 \%$ in aqueous medium [89]. Such NPs with concentration of $0.1 \mathrm{~g} / \mathrm{L}$ were added to cancer cell cultures followed with white light illumination that resulted in $45 \%$ decrease of cell number [89]. A stronger effect for the cancer cell suppression was observed for microporous Si NPs, which could enter cancer cells by endocytosis mechanism, resulting in $\sim 70 \%$ cell death [90]. Photoluminescent NPs prepared from meso- and microporous $\mathrm{Si}$ were shown to be efficient both as the photosensitizers of ${ }^{(1)} \mathrm{O}_{2}$ generation and photoluminescent labels in bioimaging in vitro. The photosensitization ability combined with the PL bioimaging property allow us to consider por-Si NPs as a potential agent for application in as-called cancer theranostics, that is, simultaneously therapy and diagnostics $[89,90]$. Note, the biological effect of photoexcited nc-Si can be related with both the photosensitized ${ }^{(1)} \mathrm{O}_{2}$ other active forms of oxygen, for example, superoxide $\left(\mathrm{O}^{-}{ }_{2}\right.$ ion $)$, whose signature was observed as spectral features of the exciton $\mathrm{PL}$ quenching at 2.0-2.2 eV [91].

While the results of fundamental studies and in vitro experiments on the ${ }^{(1)} \mathrm{O}_{2}$ photosensitization by nc-Si seem to be promising, there is a lack of in vivo tests to assess the real potential of nc-Si for PDT applications. Note, the biocompatibility and biodegradability of nc-Si and por-Si NPs [92,93] together with the photosensitization ability are well satisfied to the modern requirements for PDT application [94]. Besides nc-Si prepared from por-Si [87-90] the photosensitizing property was also reported for nc-Si prepared by laser ablation of bulk Si in water [86]. This finding indicates a possible way to obtain nc-Si, which can at as stable and efficient photosensitizers for the cancer theranostics [3].

\subsection{Si NPs with Electron Spin Centers as Potential Contrast Agents for ${ }^{1} \mathrm{H} M R I$}

Si NPs with a high number of the electron spin centers can act as contrast agents (CAs) for the conventional biomedical MRI at the proton $\left({ }^{1} \mathrm{H}\right)$ frequency [95]. The MRI contrast property of Si NPs was explained by considering the magnetic dipole-dipole interaction between electron spins on the $\mathrm{Si}$ NP's surfaces and those of surrounding protons [96] as schematically shown in Figure 36. While a solid NP itself can increase the proton spin relaxation (Figure 36a), Si NPs especially prepared from por-Si provide additional opportunities to increase the spin center density due to huge specific surface area of small nc-Si [62]. While por-Si usually contains numerous paramagnetic centers as $\mathrm{Si}$ dangling bonds (Si DBs) and their number can be significantly increased by physical and chemical treatments [77]. It was shown that Si NPs prepared from por-Si subjected to thermal annealing (TA) in vacuum could contain a lot of paramagnetic centers, which strongly enhanced the proton spin relaxation of water molecules surrounding those NPs (Figure 36b). It is well known that surface of electrochemically prepared por-Si is passivated by hydrogen atoms [77]. Because the TA treatment induces the hydrogen desorption from the NP's surface followed by creation of new Si DBs, which can be quantitatively detected by EPR technique [77]. Tailoring of the paramagnetic center number in Si NPs is a possible way to improve their CA properties for MRI applications [96].

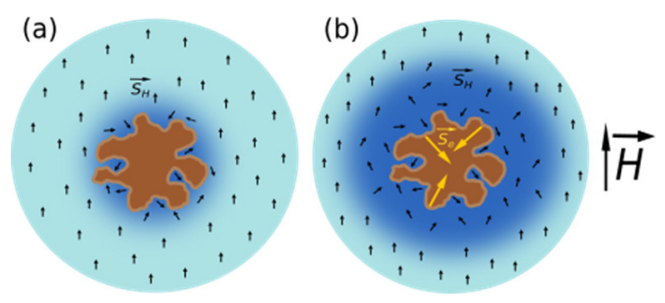

Figure 36. (a) Schematic view of the shortening of proton spin relaxation in water (blue regions) nearby a NP (brown spot) without unpaired electron spins; (b) the same scheme for the NP with numerous unpaired electron spins centers on the NP surface. Black and yellow arrows correspond to the proton and electron spins, respectively. (Reprinted from Reference [96] by permission from John Wiley and Sons (C) 2018). 
Figure 37 shows typical transients of the longitudinal (left) and transverse (right) proton magnetizations in aqueous suspensions of NPs (mean size $\sim 100 \mathrm{~nm}$ ) prepared from mesoporous (MPSi), microporous ( $\mu \mathrm{PSi}$ ) and from c-Si wafers by using high-energy ball milling of the corresponding bulk samples [96]. The MRI contrasting is caused by shortening of the relaxation times of protons in surrounding water molecules (see Figure 38). Table 1 presents data on the Si DB density and proton relaxation times for several types of Si NPs. The sample of c-Si NPs does not cause significant proton relaxation despite they have high paramagnetic centers concentration. Indeed, the surface area c-Si NPs is rather low and most of the centers are located deeply inside the NPs. NPs prepared from both MPSi and $\propto P S i$ demonstrate significant $\mathrm{T}_{2}$ shortening. Pore sizes are different in $\mu \mathrm{PSi}(\leq 2 \mathrm{~nm})$ and MPSi $(2 \mathrm{~nm}$ to $50 \mathrm{~nm}$ ) that means a bigger surface area for $\mu \mathrm{PSi}$ and a stronger interaction of water molecules with the surface of NPs.
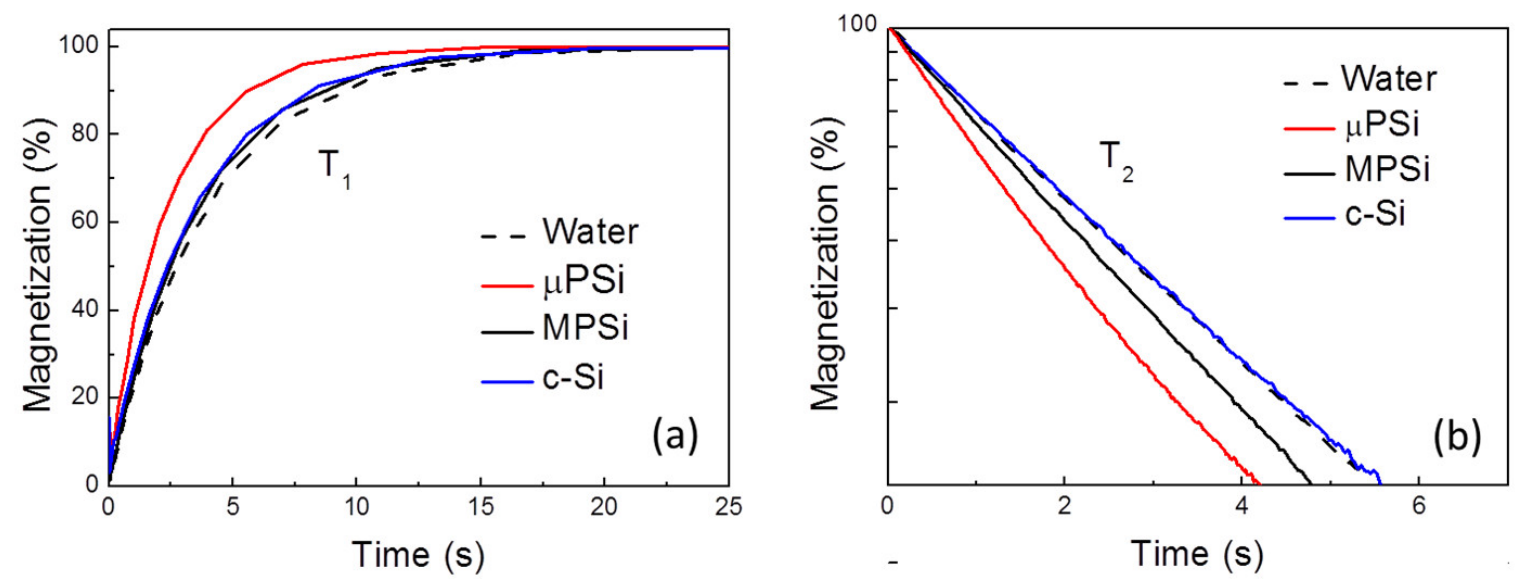

Figure 37. Transients of (a) longitudinal and (b) transverse proton magnetization of pure water (black dashed line) and aqueous suspensions of NPs (1g/L) prepared from c-Si (blue line) MPSi (black line) and $\mu$ PSi (red line). (Reprinted from Reference [96] by permission from John Wiley and Sons (C) 2018).
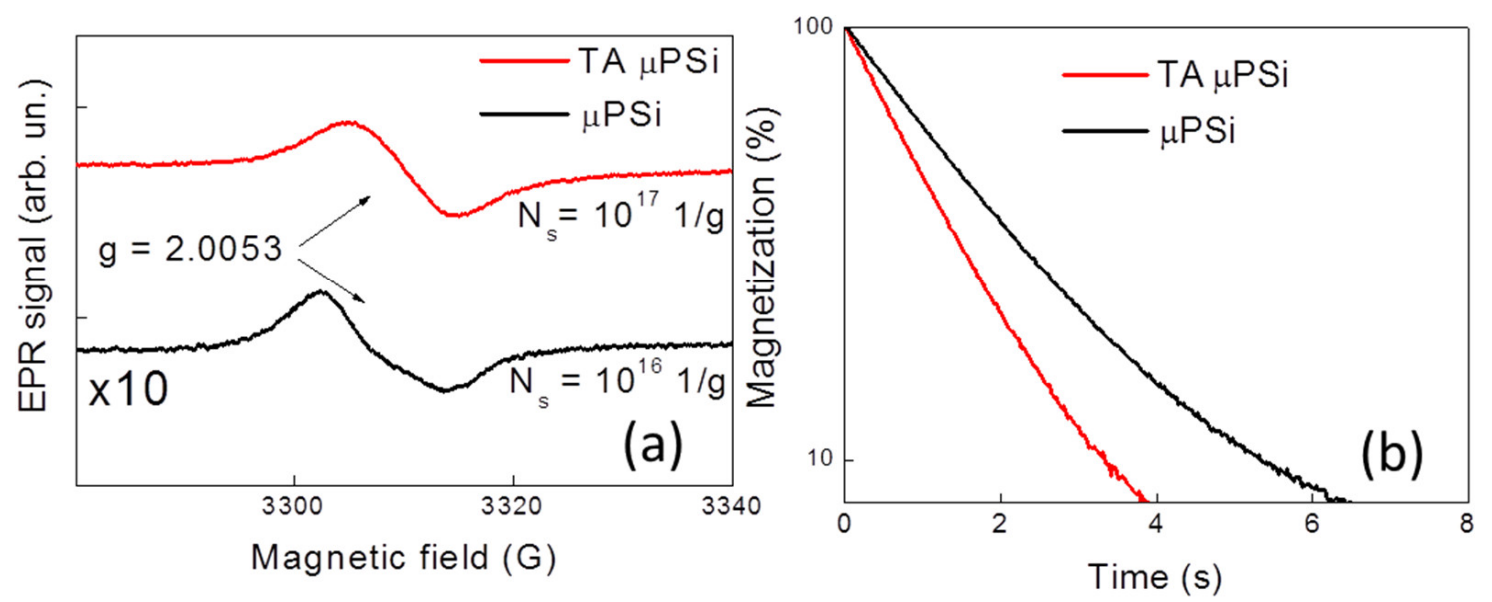

Figure 38. (a) EPR spectra of as-prepared (black line, the intensity is multiplied by 10) and thermally annealed in vacuum (red line) PSi NPs. (b) Transients of the transverse proton magnetization in aqueous suspensions of as-prepared (black line) and thermally annealed in vacuum (red line) $\mu$ PSi NPs. (Reprinted from Reference [96] by permission from John Wiley and Sons (C) 2018). 
Table 1. Electron spin concentration for several types of Si NPs and proton relaxation times $T_{1}$ and $T_{2}$ in aqueous suspensions with NP concentration of $1 \mathrm{~g} / \mathrm{L}$ and for pure water, for comparison. (Reprinted from Reference [96] by permission from John Wiley and Sons (C) 2018).

\begin{tabular}{|c|c|c|c|}
\hline \multirow{2}{*}{ Sample } & \multirow{2}{*}{$\begin{array}{l}\text { Number of Electron } \\
\text { Spin Centers }(1 / g)\end{array}$} & \multicolumn{2}{|c|}{${ }^{1} \mathrm{H}$ relaxation Times } \\
\hline & & $T_{1}(\mathrm{~s})$ & $T_{2}(\mathrm{~s})$ \\
\hline Crystalline Si (c-Si) NPs & $2 \cdot 10^{16}$ & $3.6 \pm 0.2$ & $2.7 \pm 0.2$ \\
\hline $\begin{array}{l}\text { Thermally oxidized } \\
\text { meso porous } \mathrm{Si} \\
\text { (TO MPSi) NPs }\end{array}$ & $3 \cdot 10^{14}$ & $3.5 \pm 0.2$ & $2.5 \pm 0.2$ \\
\hline $\begin{array}{l}\text { Mesoporous Si } \\
\text { (MPSi) NPs }\end{array}$ & $10^{15}$ & $3.6 \pm 0.2$ & $2.3 \pm 0.2$ \\
\hline $\begin{array}{c}\text { Microporous Si } \\
(\mu \mathrm{PSi}) \mathrm{NPs}\end{array}$ & $10^{16}$ & $3.1 \pm 0.2$ & $1.7 \pm 0.2$ \\
\hline $\begin{array}{c}\text { Microporous Si NPs after } \\
\text { thermal annealing in } \\
\text { vacuum } \\
\text { (TA } \mu \text { PSi) }\end{array}$ & $10^{17}$ & $2.7 \pm 0.2$ & $1.2 \pm 0.2$ \\
\hline Water & 0 & $4.0 \pm 0.1$ & $2.7 \pm 0.1$ \\
\hline
\end{tabular}

The EPR spectroscopy can be used to clarify both the origin of electron spin centers and to measure their concentration in Si NPs after TA. Figure 38a shows EPR spectra of $\mu$ PSi NPs before and after TA. The both spectra have the effective $g$-factor of about 2.005, which can be explained by superposition of several types of $\mathrm{Si}$ DBs [96], for example, $\mathrm{P}_{\mathrm{b}}$-centers with differently coordinated $\mathrm{Si}$ and $\mathrm{O}$ atoms $[97,98]$. The number of Si DBs after TA increases due to thermally induced desorption of hydrogen from the internal surface of $\mu$ Psi. Despite the concentration of DBs after TA increased by a factor of 10 , the $T_{2}$ relaxation time decreases only by 3 times (Figure 38b). This discrepancy can be explained by a partial passivation of Si DBs by water molecules [96]. While TA in vacuum can induce a lot of new DBs in por-Si [77], the DB concentration decreases strongly in MPSi after thermal oxidation (TO) in air at $900^{\circ} \mathrm{C}$. This effect is explained by passivation of Si DBs due to formation of a thin layer of the thermal oxide [96].

The transverse proton relaxation rate induced by Si NPs can be defined as follows [96]:

$$
R_{2} \equiv\left(T_{2}^{N P}\right)^{-1}=\left(T_{2}\right)^{-1}-\left(T_{2}^{W}\right)^{-1},
$$

where $T_{2}^{N P}$ is the time of transverse relaxation due to the presence of NPs, $T_{2}$ is the measured transverse relaxation time and $T_{2}^{W}$ is the transverse relaxation time in pure water.

Figure 39 shows that $R_{2}$ depends almost linearly on concentration of as-prepared MPSi NPs and those after TO. The latter NPs are characterized by nearly 3 times lower concentration of paramagnetic centers in comparison to the as-prepared ones (see Table 1), what causes 3 times lower $R_{2}$ in the whole range of the NP concentration (see Figure 39). This fact indicates that both types of NPs do not aggregate in highly concentrated suspensions and the pores are still open to ensure access of water molecules.

Si NP based CAs can be characterized by their specific relaxation efficiency, that is, relaxivity, which is defined as the change in relaxation rate after addition of CAs, divided to the concentration of NPs $[95,96]$. The transverse proton relaxivity can be given by the following expression:

$$
r_{2}=R_{2} / C_{N P},
$$

where $R_{2}$ is the proton relaxation rate governed by Si NPs, $C_{N P}$ is the concentration of NPs in suspensions. 


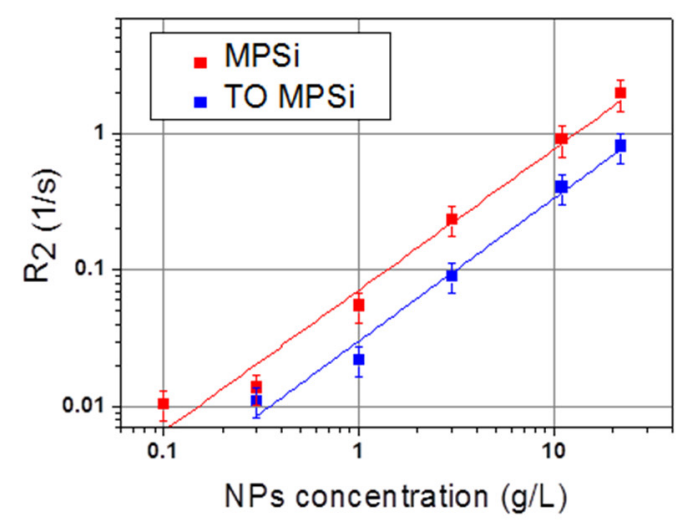

Figure 39. Transverse relaxation rate of aqueous suspensions of as-prepared (red squares) and thermally oxidized in air (blue squares) NPs prepared from MPSi versus NPs concentration. Solid lines are linear fits. (Reprinted from Reference [96] by permission from John Wiley and Sons (C 2018).

The transverse relaxivity $r_{2} \approx 0.5 \mathrm{~L} /(\mathrm{gs})$ was determined for $\mu \mathrm{PSi}$ NPs after TA that resulted in the large number of electron spin centers $\sim 10^{17} \mathrm{~g}^{-1}$ [96]. The corresponding maximal relaxivity normalized on the number of Si atoms in NPs is about $0.01 \mathrm{~L} /(\mathrm{mMs})$ that is smaller than for the standard CAs based on $\mathrm{Gd}^{3+}$ ions, which is about 4-6 L/(mMs) [99]. Note, the relaxivity of superparamagnetic iron oxide NPs is reported to be up to $10^{2}-10^{3} \mathrm{~L} /(\mathrm{mMs})$ [100]. However, specially designed porous Si NPs with required number of electron spin centers seem to be considered as potential CAs for biomedical application in MRI [96].

In order to improve the ${ }^{1} \mathrm{H}$ MRI contrasting property of Si NPs, one can use nanocomposite NPs with iron oxide [101]. Such composite Si NPs are prepared by arc-discharge plasma-assisted method in a closed-cycle inert gas ( $\mathrm{Ar}$ or $\mathrm{N}_{2}$ ) ambient at atmospheric pressure. The process consists of evaporation of high purity polycrystalline $\mathrm{Si}$ powder with addition of metallic iron powder at temperature of about $5500^{\circ} \mathrm{C}$ followed with condensation of the vapor mixture in a cooling gas stream to form polycrystalline powder with mean size of nc-Si of about 20-30 nm confirmed by XRD measurements. Despite the relatively small nanocrystal size the prepared NPs after dispersing them in aqueous media exhibit several times larger hydrodynamic sizes detected by using a DLS method (see Table 2) that indicates either polycrystalline structure of these NPs or/and their aggregation in aqueous medium [101].

Table 2. Characteristics of Si NPs with iron impurities. (Reprinted from Reference [101] by permission from John Wiley and Sons (C) 2019).

\begin{tabular}{|c|c|c|c|c|c|c|}
\hline \multirow{2}{*}{ Sample } & \multirow{2}{*}{$\begin{array}{l}\text { Fe Content, } \\
\text { at. } \%\end{array}$} & \multirow{2}{*}{$\begin{array}{l}\text { Nanocrystal Size } \\
\text { from XRD, } \mathrm{nm}\end{array}$} & \multirow{2}{*}{$\begin{array}{l}\text { Most Probable } \\
\text { Hydrodynamic Size of } \\
\text { NPs from DLS, nm }\end{array}$} & \multicolumn{2}{|c|}{ Relaxivity, L/(gs) } & \multirow{2}{*}{$\begin{array}{l}\text { RF Heating } \\
\text { Rate, } K_{\min }{ }^{-1}\end{array}$} \\
\hline & & & & $\mathbf{r}_{1}$ & $\mathbf{r}_{2}$ & \\
\hline 1 & 0.004 & $20 \pm 5$ & 130 & 0.05 & 0.47 & 6.1 \\
\hline 2 & 0.02 & $25 \pm 5$ & 50 & 0.13 & 1.38 & 12.8 \\
\hline 3 & 0.2 & $30 \pm 5$ & 120 & 0.83 & 7.30 & 1.2 \\
\hline
\end{tabular}

EPR spectra of Si NPs with iron oxide impurities (see Figure 40) consist of a broad band with effective $g$-factor $\approx 2.1$ related to strongly interacting $\mathrm{Fe}^{3+}$ ions in $\gamma-\mathrm{Fe}_{2} \mathrm{O}_{3}$ [102] and a narrow line with $g$-factor of 2.0055, which can be assigned to $\mathrm{Si} \mathrm{DBs}$ as $\mathrm{P}_{\mathrm{b}}$-centers [97]. The larger iron concentration in the samples the stronger broadening of the corresponding EPR band due to increasing the spatially inhomogeneous interactions between $\mathrm{Fe}^{3+}$ ions. As for the second line of intrinsic Si DBs they are supposed to be localized in $\mathrm{SiO}_{2}$ surface coating of the NP surfaces, which was confirmed by X-ray photoelectron spectroscopy and FTIR measurements [101]. Furthermore, HR-TEM images of iron doped Si NPs reveal numerous defects of the crystalline structure as twins and grain boundaries related to the fast cooling rate during their formation in argon flow [103]. Both the iron impurities and intrinsic defects as Si DBs are responsible for the large number of electron spin centers in such NPs [101,103]. 


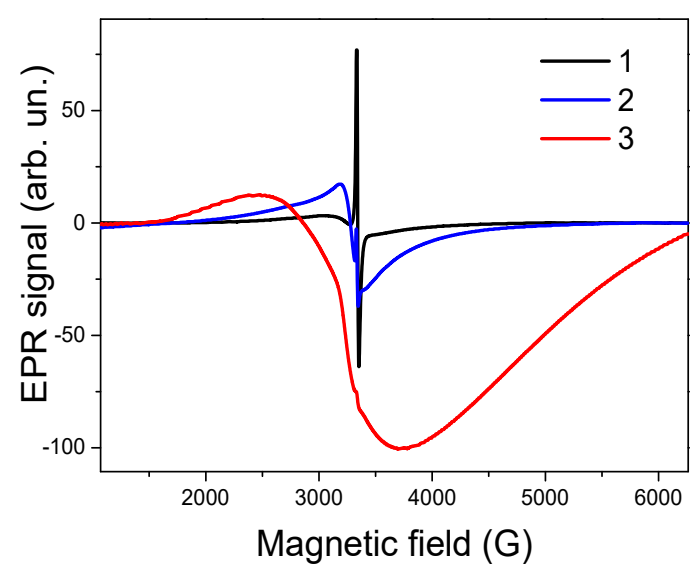

Figure 40. EPR spectra of Si NPs with different iron content increasing as $0.004,0.02$ and 0.2 at. \% for curves 1, 2 and 3, correspondingly. (Reprinted from Reference [101] by permission from John Wiley and Sons (C) 2019).

The total concentration of electron spin centers in Si NPs approaches $10^{18} \mathrm{spin} / \mathrm{g}$ [101] that makes this type of NPs to be efficient for shortening the proton spin relaxation in aqueous media as it was already demonstrated for porous Si NPs (see Figure 36). In fact, both the longitudinal and transverse relaxation times of protons in aqueous suspensions of plasma-ablated Si NPs were found to be dependent on iron content [101]. The stronger decrease of the transverse proton relaxation was detected for the samples with higher iron content up to 0.2 at $\%$. MRI experiments with phantoms showed that the latter NPs exhibited properties of efficient CAs for the ${ }^{1} \mathrm{H}$ MRI. The maximal transverse relaxivity accounted $7.3 \mathrm{~L} /(\mathrm{gs})$. The latter value corresponds to the molar relaxivity of about $200 \mathrm{~L} /(\mathrm{mMs})$ [101], which is close to that reported for CAs based on superparamagnetic iron oxide NPs [100].

The CA property of plasma-ablated Si NPs with iron oxide impurity was confirmed by experiments in vivo [101]. The experiments were carried out with grafted cancer tumor (lung carcinoma 3LL). In vivo MRI visualization of mice with tumor was carried out by using a protocol, which included the following steps: (i) obtaining whole body PD-weighted MRI before injection the NP suspension; (ii) obtaining whole body PD-weighted MRI $20 \mathrm{~min}$ after intratumorally injection of $0.5 \mathrm{~mL}$ suspension of Si NPs with concentration of $0.5 \mathrm{mg} / \mathrm{mL}$. It was found that tumor area with injected NPs became darker and have more clear boundaries (see Figure 41). The MRI study of the mice for the time period of $24 \mathrm{~h}$ after injection of Si NPs showed a decrease of the MRI contrast. This fact can be explained by biodegradation of Si NPs similarly to that of pure Si NPs [101].

Moreover, aqueous suspensions of Si NPs were explored as efficient sensitizers of electromagnetic radiofrequency (RF) hyperthermia, which is a prospective approach for mild cancer therapy with non-magnetic Si NPs. In Reference [101] the stronger heating rate was reported for iron-doped Si NPs with hydrodynamic size $50 \mathrm{~nm}$ (see Table 2). The obtained results indicate possible ways for applications of Si-based NPs in the MRI diagnostics and RF therapy of cancer, that is, theranostics. Additional promises in biomedical applications of Si NPs can arise from multinuclear MRI diagnostics by using both the standard ${ }^{1} \mathrm{H}$ mode and hyperpolarized ${ }^{29} \mathrm{Si} \mathrm{NPs} / \mathrm{MPs}$ (see Section 2) as well as other NPs with conventional biomedical nuclei, for example, ${ }^{13} \mathrm{C},{ }^{19} \mathrm{~F}$ and so forth (see, for example, Reference [104]).

Besides the MRI diagnostic modality, it was recently demonstrated the possibility to use Si NPs with iron impurities to suppress the cancer tumor growth in vivo [103]. The intratumoral injection and intravenous administration of iron-doped Si NPs resulted in a decrease of the tumor volume by $32 \%$ and $23 \%$, respectively. While the intratumoral injection induces the stronger antitumor effect, the observed decrease in tumor volume after intravenous administration confirms accumulation of Si NPs in the tumor due to as-called enhanced permeability and retention effect, which is well known for various NPs [105]. It was found that the antitumor effect of iron-doped Si NPs correlate with lifetime 
of laboratory animals [103]. Figure 42 shows temporal dependences of the number of mice with cancer tumor after intravenous and intratumoral administration of NPs. The average lifetime of laboratory animals of the experimental group was about 30\% longer than for the control one. The observed antitumor effect of iron-doped Si NPs can be related to dissolution of Si NPs accumulated in tumor followed with release of iron ions, which induce reactive oxygen species with high toxicity for cancer cells [103].

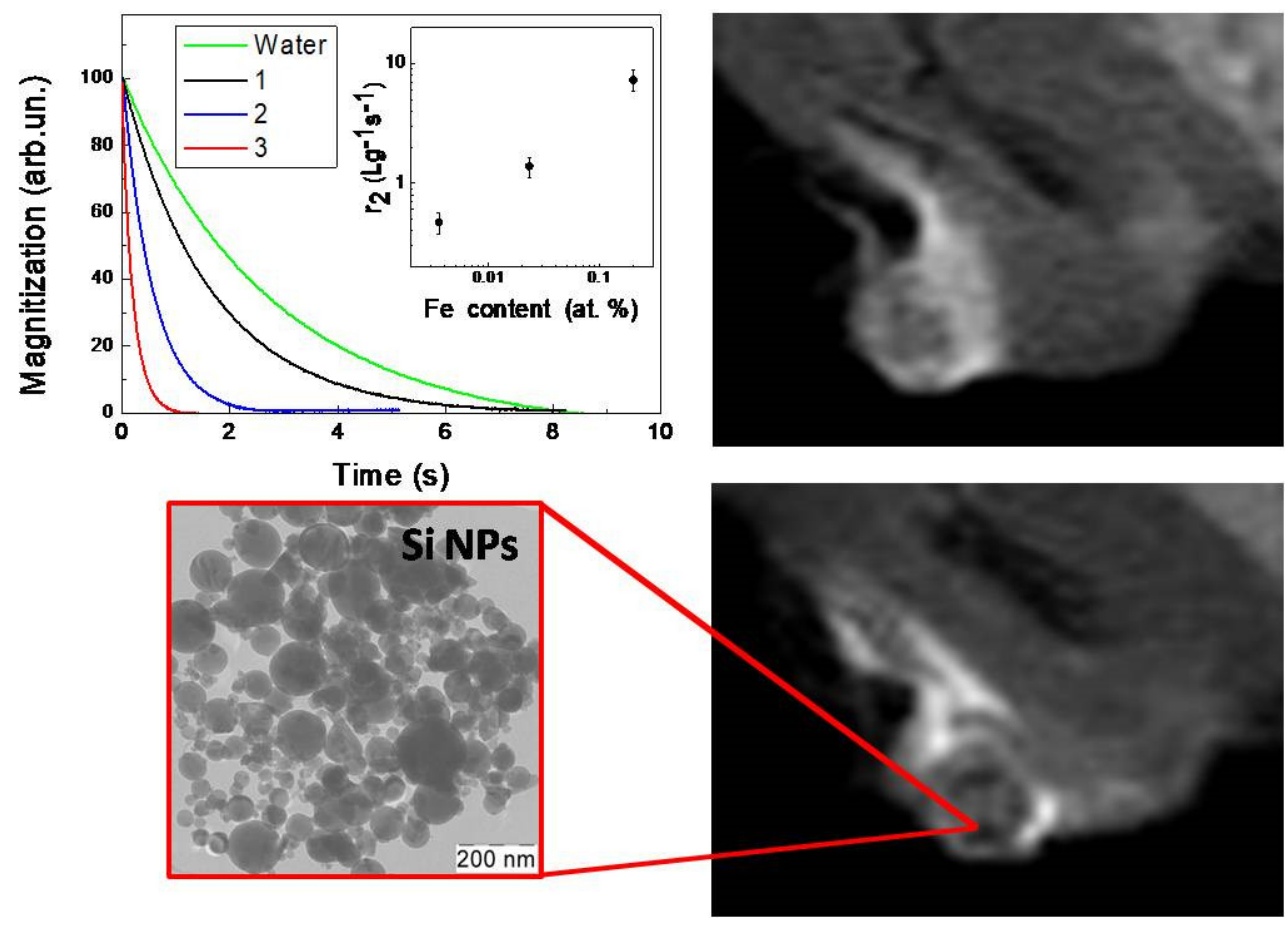

Figure 41. Transients of the proton magnetization (inset shows the transverse relaxivity vs the iron content), typical transmission electron microscopy (TEM) image of Si NPs with 0.2 at $\%$ of iron impurities and MRI images of cancer tumor before (upper image) and after (down image) injection of such NPs. (Reprinted from Reference [101] by permission from John Wiley and Sons (C) 2019).

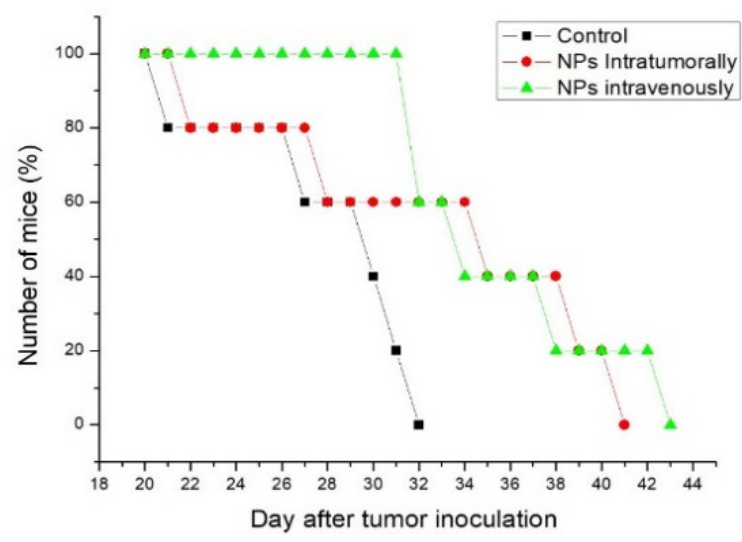

Figure 42. Number of living mice with Lewis lung carcinoma tumor after intratumoral (red circles) and intravenous administration (green triangles) of Si NPs with 0.2 at $\%$ of iron impurities and for the control group (black squares) vs time after tumor inoculation. (Reprinted from Reference [103] by permission from World Scientific Publishing (C) 2020).

\section{Summary and Outlook}

Spin-dependent phenomena in semiconductor NPs are overviewed starting from the basic properties of solid-state semiconductor systems, which depend on preferential orientation of electron 
and nuclear spins therein, to the recent advances in the development and characterization of semiconductor (mainly, silicon) NPs with spin-dependent properties that are promising for versatile biomedical applications.

A challenging task to control the nuclear-spin polarization in solids has been successfully accomplished in the pioneering work by G. Lampel on the DNP by optical pumping in c-Si. The hyperfine dipolar interaction between the electron and nuclear spins caused an appreciable polarization of the nuclear spins due to the optically induced saturation of the electron spins. Starting from then, the role of the hyperfine interaction was carefully explored to realize the hyperpolarized nuclear states in semiconductor NPs and compound MPs. The hyperpolarization efficiency of ${ }^{29} \mathrm{Si}$ was found to depend on the nuclear relaxation time controlled by the density of electron spins. The nuclear longitudinal relaxation time was shown to shorten with increasing the charge-carrier concentration in semiconductor. The nuclear-spin relaxation in Si NPs underwent a strong shortening with decreasing the mean size of NPs, while surface-passivated Si NPs exhibited the relaxation time from $10^{2} \mathrm{~s}$ to $10^{3} \mathrm{~s}$. In contrast to the nuclear relaxation, the spatial confinement of charge carriers (electrons and holes) in nanostructures significantly increased the electron spin-lattice relaxation time. A transition from itinerant to fully-localized electrons, while inducing hyperfine dephasing, was beneficial in quenching the electron spin-lattice relaxation. The phenomenon of DNP of ${ }^{29} \mathrm{Si}$ in Si NPs and compound MPs was shown to open fascinating prospects for creation of new efficient contrast agents for MRI. The most efficient DNP was achieved for Si MPs, which consisted of hundreds of nanometer-size NPs, what ensured the optimal ratio between nuclear polarization/depolarization as it was confirmed by experiments in vivo.

Besides the nuclear polarization, the electron-spin related phenomena were shown to be very promising for MRI applications. The experimental results confirmed a key role of unpaired electron spins as Si DBs in porous Si MPs and NPs for the enhancement of the proton spin relaxation in aqueous suspensions. The samples of nonporous crystalline Si NPs exhibited a relatively weak decrease of both longitudinal and transverse relaxation times because of low efficiency of the interaction of their unpaired electron spins with proton spins in surrounding water molecules. On the one hand, the thermally oxidized mesoporous Si NPs did not induce a valuable rate of the proton-spin relaxation due to the low density of Si DBs. On the other hand, the vacuum annealed microporous Si NPs exhibited a strong decrease of the relaxation times, especially the transverse one by 2 to 3 times for the NP concentration of the order of $1 \mathrm{mg} / \mathrm{mL}$. The transverse relaxation rate was found to depend nearly linearly on the concentration of porous Si NPs in suspensions ranging from 0.1 to $20 \mathrm{~g} / \mathrm{L}$. The relaxation rate induced by porous $\mathrm{Si}$ NPs was approximately proportional to the number of Si DBs on their surfaces, what indicated a way to enhance the proton relaxation via the interaction with unpaired localized electron spins of Si NPs. The transverse specific relaxation rate (relaxivity) of thermally annealed microporous Si NPs is estimated to be $0.5 \mathrm{~L} /(\mathrm{gs})$ and for plasma-ablated Si NPs with 0.2 at $\%$ of iron oxide the corresponding relaxivity is of the order of $10 \mathrm{~L} /(\mathrm{gs})$, which is already promising for MRI applications. The relaxivity of Si NPs can be further improved by using physical and chemical treatments, which will lead to increasing the number of paramagnetic centers in Si NPs that ensures further progress in the design and validation of those NPs as contrast agents for MRI. It is worth noting that both the ${ }^{1} \mathrm{H}$ MRI contrast properties of Si NPs and their hyperpolarizing properties allow us to consider such NPs as binuclear CAs, which are simultaneously detected at the proton and ${ }^{29} \mathrm{Si}$ frequencies, what is a promising direction in MRI.

In addition to the diagnostic modality of the spin-dependent properties of Si NPs, it was found that the triplet excitons confined in Si NPs of sizes below $10 \mathrm{~nm}$ could transfer their energy to molecular oxygen that resulted in generation of highly chemically reactive singlet oxygen promising for the photodynamic therapy of cancer. The energy-transfer process depended on the magnetic field that indicated the spin-related origin of the singlet-oxygen photosensitization. The photosensitizing properties of NPs made from porous Si were tested in experiments with photoinduced destruction 
of cancer cells in vitro. Further experiments are ongoing to advance Si-NP-based contrast agents for clinical applications in both the MRI diagnostics and the mild therapy of cancer.

Author Contributions: Conceptualization and methodology, V.M.F. and V.Y.T.; writing-original draft preparation of Sections 1 and 2, V.M.F.; writing-original draft preparation of Sections 3 and 4, V.Y.T.; writing-review and editing, V.M.F. and V.Y.T.; project administration, V.M.F.; funding acquisition, V.Y.T. All authors have read and agreed to the published version of the manuscript.

Funding: The work was partially funded by the National Research Nuclear University MEPhI Academic Excellence Project (Contract No. 02.a03.21.0005). V. Yu. T. was funded by the Russian Science Foundation (Project No. 19-72-30012).

Acknowledgments: The authors are grateful to Yulia V. Kargina for her assistance in the manuscript preparation. V. M. F. is grateful to Fabio Pezzoli and Randall Q. Snurr for fruitful interactions in the course of preparation of the present review paper.

Conflicts of Interest: The authors declare no conflict of interest.

\section{References}

1. Luu, Q.S.; Kim, J.; Jo, D.; Jeong, J.; Lee, Y. Applications and perspective of silicon particles in hyperpolarized ${ }^{29}$ Si magnetic resonance imaging. Appl. Spectrosc. Rev. 2020, 55, 476-490. [CrossRef]

2. Timoshenko, V.Y. Porous Silicon in Photodynamic and Photothermal Therapy. In Handbook of Porous Silicon, 2nd ed.; Canham, L., Ed.; Springer: Berlin/Heidelberg, Germany, 2018; pp. 1461-1469. [CrossRef]

3. Kabashin, A.V.; Timoshenko, V.Y. What theranostic applications could ultrapure laser-synthesized Si nanoparticles have in cancer? Nanomedicine 2016, 11, 2247-2250. [CrossRef]

4. Zinovieva, A.F.; Nikiforov, A.I.; Timofeev, V.A.; Nenashev, A.V.; Dvurechenskii, A.V.; Kulik, L.V. Electron localization in $\mathrm{Ge} / \mathrm{Si}$ heterostructures with double quantum dots detected by an electron spin resonance method. Phys. Rev. B 2013, 88, 235308. [CrossRef]

5. Lipps, F.; Pezzoli, F.; Stoffel, M.; Deneke, C.; Thomas, J.; Rastelli, A.; Kataev, V.; Schmidt, O.G.; Büchner, B. Electron spin resonance study of Si/SiGe quantum dots. Phys. Rev. B 2010, 81, 125312. [CrossRef]

6. Giorgioni, A.; Paleari, S.; Cecchi, S.; Vitiello, E.; Grilli, E.; Isella, G.; Jantsch, W.; Fanciulli, M.; Pezzoli, F. Strong confinement-induced engineering of the $\mathrm{g}$ factor and lifetime of conduction electron spins in Ge quantum wells. Nat. Commun. 2016, 7, 13886. Available online: https://www.nature.com/articles/ncomms13886 (accessed on 17 July 2020). [CrossRef]

7. Khaetskii, A.V.; Nazarov, Y.V. Spin-flip transitions between Zeeman sublevels in semiconductor quantum dots. Phys. Rev. B 2001, 64, 125316. [CrossRef]

8. Alcalde, A.M.; Marques, G.E. Electron spin-phonon relaxation in quantum dots. Braz. J. Phys. 2004, 34, 705-707. Available online: http://www.sbfisica.org.br/bjp/files/v34_705.pdf (accessed on 17 July 2020). [CrossRef]

9. Abragam, A.; Goldman, M. Principles of dynamic nuclear polarisation. Rep. Prog. Phys. 1978, 41, $395-467$. [CrossRef]

10. Kwiatkowski, G.; Polyhach, Y.; Jähnig, F.; Shiroka, T.; Starsich, F.H.L.; Ernst, M.; Kozerke, S. Exploiting Endogenous Surface Defects for Dynamic Nuclear Polarization of Silicon Micro- and Nanoparticles. J. Phys. Chem. C 2018, 122, 25668-25680. [CrossRef]

11. Aptekar, J.W.; Cassidy, M.C.; Johnson, A.C.; Barton, R.A.; Lee, M.; Ogier, A.C.; Vo, C.; Anahtar, M.N.; Ren, Y.; Bhatia, S.N.; et al. Silicon Nanoparticles as Hyperpolarized Magnetic Resonance Imaging Agents. ACS Nano 2009, 3, 4003-4008. [CrossRef]

12. Abragam, A. The Principles of Nuclear Magnetism; Oxford University Press: Oxford, UK, 1961.

13. Jansen, R. Silicon spintronics. Nat. Mater. 2012, 11, 400-408. [CrossRef] [PubMed]

14. Lampel, G. Nuclear Dynamic Polarization by Optical Electronic Saturation and Optical Pumping in Semiconductors. Phys. Rev. Lett. 1968, 20, 491-493. [CrossRef]

15. Lampel, G. Relaxation Nucléaire dans le Silicium à $77^{\circ} \mathrm{K}$ et Polarisation Dynamique par Pompage Optique. Ph.D. Thesis, Université de Paris, Paris, France, 1968.

16. Overhauser, A.W. Polarization of Nuclei in Metals. Phys. Rev. 1953, 92, 411-415. [CrossRef]

17. Kittel, C. Introduction to Solid State Physics; John Wiley \& Sons: New York, NY, USA, 1996. 
18. Shulman, R.G.; Wyluda, B.J. Nuclear magnetic resonance of ${ }^{29} \mathrm{Si}$ in $\mathrm{n}$ - and p-type silicon. Phys. Rev. 1956, 103, 1127-1129. [CrossRef]

19. Bloembergen, N. Nuclear magnetic relaxation in semiconductors. Physica 1954, 20, 1130-1133. [CrossRef]

20. Lee, M.; Cassidy, M.C.; Ramanathan, C.; Marcus, C.M. Decay of nuclear hyperpolarization in silicon microparticles. Phys. Rev. B 2011, 84, 035304. [CrossRef]

21. Dyakonov, M.I.; Perel, V.I. Theory of Optical Spin Orientation of Electrons and Nuclei in Semiconductors. In Optical Orientation; Meier, F., Zakharchenya, B.P., Eds.; Elsevier: Amsterdam, The Netherlands, 1984. [CrossRef]

22. Chekhovich, E.A.; Glazov, M.M.; Krysa, A.; Hopkinson, M.; Senellart, P.; Lemaître, A.; Skolnick, M.S.; Tartakovskii, A.I. Element-sensitive measurement of the hole-nuclear spin interaction in quantum dots. Nat. Phys. 2012, 9, 74-78. [CrossRef]

23. Overhauser, A.W. Paramagnetic Relaxation in Metals. Phys. Rev. 1953, 89, 689-700. [CrossRef]

24. Li, P.; Dery, H. Theory of Spin-Dependent Phonon-Assisted Optical Transitions in Silicon. Phys. Rev. Lett. 2010, 105, 1-4. [CrossRef]

25. Cassidy, M.C.; Chan, H.R.; Ross, B.D.; Bhattacharya, P.K.; Marcus, C.M. In vivo magnetic resonance imaging of hyperpolarized silicon particles. Nat. Nanotechnol. 2013, 8, 363-368. [CrossRef]

26. Atkins, T.M.; Cassidy, M.C.; Lee, M.; Ganguly, S.; Marcus, C.M.; Kauzlarich, S.M. Synthesis of Long $T_{1}$ Silicon Nanoparticles for Hyperpolarized ${ }^{29}$ Si Magnetic Resonance Imaging. ACS Nano 2013, 7, 1609-1617. [CrossRef]

27. Bagraev, N.T.; Vlasenko, L.S.; Zhitnikov, R.A. Optical orientation of $\mathrm{Si}^{29}$ nuclei in n-type silicon and its dependence on the pumping light intensity. Sov. Phys. JETP 1976, 44, 500-504.

28. Bagraev, N.T.; Vlasenko, L.S.; Zhitnikov, R.A. Influence of the depth of location of donor levels on the degree of optical orientation of ${ }^{29}$ Si nuclei in silicon. Sov. Phys. JETP Lett. 1976, 24, 366-368.

29. Verhulst, A.S.; Rau, I.G.; Yamamoto, Y.; Itoh, K.M. Optical pumping of $\mathrm{Si}^{29}$ nuclear spins in bulk silicon at high magnetic field and liquid helium temperature. Phys. Rev. B 2005, 71, 1-10. [CrossRef]

30. Ekimov, A.I.; Safarov, V.I. Optical detection of dynamic polarization of nuclei in semiconductors. JETP Lett. $1972,15,179-181$.

31. Tycko, R. Optical pumping in indium phosphide: ${ }^{31} \mathrm{P}$ NMR measurements and potential for signal enhancement in biological solid state NMR. Solid State Nucl. Magn. Reson. 1998, 11, 1-9. [CrossRef]

32. Pietraß, T.; Tomaselli, M. Optically pumped NMR in CdS single crystals. Phys. Rev. B 1999, 59, $1986-1989$. [CrossRef]

33. Cheng, J.L.; Rioux, J.; Fabian, J.; Sipe, J.E. Theory of optical spin orientation in silicon. Phys. Rev. B 2011, 83, 1-15. [CrossRef]

34. Cheng, J.L.; Rioux, J.; Sipe, J.E. Two-photon indirect optical injection and two-color coherent control in bulk silicon. Phys. Rev. B 2011, 84, 1-13. [CrossRef]

35. Guite, C.; Venkataraman, V. Measurement of Electron Spin Lifetime and Optical Orientation Efficiency in Germanium Using Electrical Detection of Radio Frequency Modulated Spin Polarization. Phys. Rev. Lett. 2011, 107, 1-4. [CrossRef] [PubMed]

36. Pezzoli, F.; Bottegoni, F.; Trivedi, D.; Ciccacci, F.; Giorgioni, A.; Li, P.; Cecchi, S.; Grilli, E.; Song, Y.; Guzzi, M.; et al. Optical Spin Injection and Spin Lifetime in Ge Heterostructures. Phys. Rev. Lett. 2012, 108, 1-5. [CrossRef] [PubMed]

37. Giorgioni, A.; Vitiello, E.; Grilli, E.; Guzzi, M.; Pezzoli, F. Valley-dependent spin polarization and long-lived electron spins in germanium. Appl. Phys. Lett. 2014, 105, 152404. [CrossRef]

38. Li, P.; Trivedi, D.; Dery, H. Spin-dependent optical properties in strained silicon and germanium. Phys. Rev. $B$ 2013, 87, 1-15. [CrossRef]

39. Liu, Z.; Nestoklon, M.O.; Cheng, J.L.; Ivchenko, E.L.; Wu, M.W. Spin-dependent intravalley and intervalley electron-phonon scatterings in germanium. Phys. Solid State 2013, 55, 1619-1734. [CrossRef]

40. Sircar, N.; Bougeard, D. Experimental investigation of the optical spin-selection rules in bulk $\mathrm{Si}$ and $\mathrm{Ge} / \mathrm{Si}$ quantum dots. Phys. Rev. B 2014, 89, 1-5. [CrossRef]

41. Akimoto, I.; Naka, N. Two optical routes of cold carrier injection in silicon revealed by time-resolved excitation spectroscopy. Appl. Phys. Express 2017, 10,1-4. [CrossRef]

42. Bottegoni, F.; Zucchetti, C.; Ciccacci, F.; Finazzi, M.; Isella, G. Optical generation of pure spin currents at the indirect gap of bulk Si. Appl. Phys. Lett. 2017, 110, 1-5. [CrossRef] 
43. Cushing, S.K.; Zürch, M.; Kraus, P.M.; Carneiro, L.M.; Lee, A.; Chang, H.-T.; Kaplan, C.J.; Leone, S.R. Hot phonon and carrier relaxation in $\mathrm{Si}(100)$ determined by transient extreme ultraviolet spectroscopy. Struct. Dyn. 2018, 5, 1-20. [CrossRef]

44. Zucchetti, C.; Bottegoni, F.; Isella, G.; Finazzi, M.; Rortais, F.; Vergnaud, C.; Widiez, J.; Jamet, M.; Ciccacci, F. Spin-to-charge conversion for hot photoexcited electrons in germanium. Phys. Rev. B 2018, 97, 1-7. [CrossRef]

45. De Cesari, S.; Balocchi, A.; Vitiello, E.; Jahandar, P.; Grilli, E.; Amand, T.; Marie, X.; Myronov, M.; Pezzoli, F. Spin-coherent dynamics and carrier lifetime in strained $\mathrm{Ge}_{1-\mathrm{x}} \mathrm{Sn}_{\mathrm{x}}$ semiconductors on silicon. Phys. Rev. B 2019, 99, 1-9. [CrossRef]

46. Ardenkjaer-Larsen, J.H.; Fridlund, B.; Gram, A.; Hansson, G.; Lennart Hansson, L.; Lerche, M.H.; Servin, R.; Thaning, M.; Golman, K. Increase in signal-to-noise ratio of $>10,000$ times in liquid-state NMR. Proc. Natl. Acad. Sci. USA 2003, 100, 10158-10163. [CrossRef] [PubMed]

47. Fujiwara, T.; Ramamoorthy, A. How far can the sensitivity of NMR be increased? In Annual Reports on NMR Spectroscopy; Academic Press: Cambridge, MA, USA, 2006; Volume 58, pp. 155-175. [CrossRef]

48. Van Kesteren, H.W.; Wenckebach, W.T.; Schmidt, J. Production of High, Long-Lasting, Dynamic Proton Polarization by Way of Photoexcited Triplet States. Phys. Rev. Lett. 1985, 55, 1642-1644. [CrossRef] [PubMed]

49. Lelli, M.; Gajan, D.; Lesage, A.; Caporini, M.A.; Vitzthum, V.; Miéville, P.; Héroguel, F.; Rascón, F.; Roussey, A.; Thieuleux, C.; et al. Fast Characterization of Functionalized Silica Materials by Silicon-29 Surface-Enhanced NMR Spectroscopy Using Dynamic Nuclear Polarization. J. Am. Chem. Soc. 2011, 133, 2104-2107. [CrossRef]

50. Casabianca, L.B.; Shames, A.I.; Panich, A.M.; Shenderova, O.; Frydman, L. Factors Affecting DNP NMR in Polycrystalline Diamond Samples. J. Phys. Chem. C 2011, 115, 19041-19048. [CrossRef]

51. Dementyev, A.E.; Cory, D.G.; Ramanathan, C. Dynamic Nuclear Polarization in Silicon Microparticles. Phys. Rev. Lett. 2008, 100, 1-4. [CrossRef] [PubMed]

52. Kwiatkowski, G.; Jähnig, F.; Steinhauser, J.; Wespi, P.; Ernst, M.; Kozerke, S. Nanometer size silicon particles for hyperpolarized MRI. Sci. Rep. 2017, 7, 1-6. Available online: https://www.nature.com/articles/s41598017-08709-0 (accessed on 17 July 2020). [CrossRef]

53. Whiting, N.; Hu, J.; Shah, J.V.; Cassidy, M.C.; Cressman, E.; Millward, S.W.; Menter, D.G.; Marcus, C.M.; Bhattacharya, P.K. Real-Time MRI-Guided Catheter Tracking Using Hyperpolarized Silicon Particles. Sci. Rep. 2015, 5, 1-8. [CrossRef]

54. Whiting, N.; Hu, J.; Zacharias, N.M.; Lokesh, G.L.; Volk, D.E.; Menter, D.G.; Rupaimoole, R.; Previs, R.; Sood, A.K.; Bhattacharya, P. Developing hyperpolarized silicon particles for In Vivo MRI targeting of ovarian cancer. J. Med. Imaging 2016, 3, 1-9. [CrossRef]

55. Seo, H.; Choi, I.; Whiting, N.; Hu, J.; Luu, Q.S.; Pudakalakatti, S.; McCowan, C.; Kim, Y.; Zacharias, N.; Lee, Y.; et al. Hyperpolarized Porous Silicon Nanoparticles: Potential Theragnostic Material for ${ }^{29}$ Si Magnetic Resonance Imaging. ChemPhysChem 2018, 19, 2143-2147. [CrossRef]

56. Hu, J.; Whiting, N.; Bhattacharya, P.K. Hyperpolarization of Silicon Nanoparticles with TEMPO Radicals. J. Phys. Chem. C 2018, 122, 10575-10581. [CrossRef]

57. Sanders, G.D.; Chang, Y.-C. Theory of optical properties of quantum wires in porous silicon. Phys. Rev. B 1992, 45, 9202-9213. [CrossRef] [PubMed]

58. Delley, B.; Steigmeier, E.F. Size dependence of band gaps in silicon nanostructures. Appl. Phys. Lett. 1995, 67, 2370-2372. [CrossRef]

59. John, G.J.; Singh, V.A. Porous silicon: Theoretical studies. Phys. Rep. 1995, 263, 93-151. [CrossRef]

60. Calcott, P.D.J.; Nash, K.J.; Canham, L.T.; Kane, M.J.; Brumhead, D. Identification of radiative transitions in highly porous silicon. J. Phys. Condens. Matter 1993, 5, L91-L98. [CrossRef]

61. Kovalev, D.; Heckler, H.; Polisski, G.; Koch, F. Optical Properties of Si Nanocrystals. Phys. Status Solidi B 1999, 215, 871-932. [CrossRef]

62. Bisi, O.; Ossicini, S.; Pavesi, L. Porous silicon: A quantum sponge structure for silicon based optoelectronics. Surf. Sci. Rep. 2000, 38, 1-126. [CrossRef]

63. Moser, J.G. Photodynamic Tumor Therapy: 2nd and 3rd Generation Photosensitizers; Harwood Academic Publushers: Amsterdam, The Netherlands, 1998.

64. Kovalev, D.; Gross, E.; Künzner, N.; Koch, F.; Timoshenko, V.Y.; Fujii, M. Resonant Electronic Energy Transfer from Excitons Confined in Silicon Nanocrystals to Oxygen Molecules. Phys. Rev. Lett. 2002, 89, 1-4. [CrossRef] 
65. Gross, E.; Kovalev, D.; Künzner, N.; Diener, J.; Koch, F.; Timoshenko, V.Y.; Fujii, M. Spectrally resolved electronic energy transfer from silicon nanocrystals to molecular oxygen mediated by direct electron exchange. Phys. Rev. B 2003, 68, 1-11. [CrossRef]

66. Turro, N.J. Modern Molecular Photochemistry; University Science Books: Mill Valley, CA, USA, 1991.

67. Kearns, D.R. Physical and chemical properties of singlet molecular oxygen. Chem. Rev. 1971, 71, $395-427$. [CrossRef]

68. Arnold, S.J.; Kubo, M.; Ogryzlo, E.A. Relaxation and Reactivity of Singlet Oxygen. Adv. Chem. 1968, 77, 133-142. [CrossRef]

69. Krasnovsky, A.A.; Kubo, M.; Ogryzlo, A. Singlet molecular oxygen in photobiochemical systems: IR phosphorescence studies. Membr. Cell Biol. 1998, 12, 665-690.

70. Fujii, M.; Minobe, S.; Usui, M.; Hayashi, S.; Gross, E.; Diener, J.; Kovalev, D. Singlet oxygen formation by porous Si in solution. Phys. Rev. B 2004, 70, 085311. [CrossRef]

71. Kovalev, D.; Fujii, M. Silicon Nanocrystals: Photosensitizers for Oxygen Molecules. Adv. Mater. 2005, 17, 2531-2544. [CrossRef]

72. Chirvony, V.; Bolotin, V.; Matveeva, E.; Parkhutik, V. Fluorescence and $1 \mathrm{O} 2$ generation properties of porphyrin molecules immobilized in oxidized nano-porous silicon matrix. J. Photochem. Photobiol. A Chem. 2006, 181, 106-113. [CrossRef]

73. Gongalsky, M.; Konstantinova, E.A.; Osminkina, L.; Timoshenko, V.Y. Detection of singlet oxygen in photoexcited porous silicon nanocrystals by photoluminescence measurements. Semiconductors 2010, 44, 89-92. [CrossRef]

74. Konstantinova, E.A.; Demin, V.A.; Timoshenko, V.Y.; Kashkarov, P.K. EPR diagnostics of the photosensitized generation of singlet oxygen on the surface of silicon nanocrystals. JETP Lett. 2007, 85, 59-62. [CrossRef]

75. Konstantinova, E.A.; Demin, V.A.; Timoshenko, V.Y. Investigation of the generation of singlet oxygen in ensembles of photoexcited silicon nanocrystals by electron paramagnetic resonance spectroscopy. J. Exp. Theor. Phys. 2008, 107, 473-481. [CrossRef]

76. Fujii, M.; Usui, M.; Hayashi, S.; Gross, E.; Kovalev, D.; Künzner, N.; Diener, J.; Timoshenko, V.Y. Singlet oxygen formation by porous Si in solution. Phys. Status Solidi A 2005, 202, 1385-1389. [CrossRef]

77. Cullis, A.G.; Canham, L.T.; Calcott, P.D.J. The structural and luminescence properties of porous silicon. J. Appl. Phys. 1997, 82, 909-965. [CrossRef]

78. Dexter, D.L. A Theory of Sensitized Luminescence in Solids. J. Chem. Phys. 1953, 21, 836. [CrossRef]

79. Palenov, D.A.; Zhigunov, D.M.; Shalygina, O.A.; Kashkarov, P.K.; Timoshenko, V.Y. Specific features of dissipation of electronic excitation energy in coupled molecular solid systems based on silicon nanocrystals on intense optical pumping. Semiconductors 2007, 41, 1351-1355. [CrossRef]

80. Delerue, C.; Lannoo, M.; Allan, G.; Martin, E.; Mihalcescu, I.; Vial, J.C.; Romestain, R.; Muller, F.; Bsiesy, A. Auger and Coulomb Charging Effects in Semiconductor Nanocrystallites. Phys. Rev. Lett. 1995, 75, $2228-2231$. [CrossRef] [PubMed]

81. Kovalev, D.; Gross, E.; Diener, J.; Timoshenko, V.Y.; Fujii, M. Photodegradation of porous silicon induced by photogenerated singlet oxygen molecules. Appl. Phys. Lett. 2004, 85, 3590. [CrossRef]

82. Langmuir, I. The constitution and fundamental properties of solids and liquids. Part I. Solids. J. Am. Chem. Soc. 1916, 38, 2221-2295. [CrossRef]

83. Ryabchikov, Y.V.; Belogorokhov, I.A.; Vorontsov, A.S.; Osminkina, L.; Timoshenko, V.Y.; Kashkarov, P.K. Dependence of the singlet oxygen photosensitization efficiency on morphology of porous silicon. Phys. Status Solidi A 2007, 204, 1271-1275. [CrossRef]

84. Konstantinova, E.; Demin, V.; Vorontzov, A.; Ryabchikov, Y.V.; Belogorokhov, I.; Osminkina, L.; Forsh, P.; Kashkarov, P.; Timoshenko, V.Y. Electron-paramagnetic resonance and photoluminescence study of Si nanocrystals-photosensitizers of singlet oxygen molecules. J. Non Cryst. Solids 2006, 352, 1156-1159. [CrossRef]

85. Cantin, J.L.; Schoisswohl, M.; Van Bardeleben, H.J.; Zoubir, N.H.; Vergnat, M. Electron-paramagnetic-resonance study of the microscopic structure of the Si(001)-SiO2 interface. Phys. Rev. B 1995, 52, R11599-R11602. [CrossRef]

86. Rioux, D.; Laferrière, M.; Douplik, A.; Shah, D.; Lilge, L.; Kabashin, A.V.; Meunier, M.M. Silicon nanoparticles produced by femtosecond laser ablation in water as novel contamination-free photosensitizers. J. Biomed. Opt. 2009, 14, 1-4. [CrossRef]

87. Timoshenko, V.Y.; Kudryavtsev, A.A.; Osminkina, L.; Vorontsov, A.S.; Ryabchikov, Y.V.; Belogorokhov, I.A.; Kovalev, D.; Kashkarov, P.K. Silicon nanocrystals as photosensitizers of active oxygen for biomedical applications. JETP Lett. 2006, 83, 423-426. [CrossRef] 
88. Timoshenko, V.Y.; Osminkina, L.; Vorontsov, A.S.; Ryabchikov, Y.V.; Gongalsky, M.; Efimova, A.I.; Konstantinova, E.A.; Bazylenko, T.Y.; Kashkarov, P.K.; Kudriavtsev, A.A. Silicon nanocrystals as efficient photosensitizer of singlet oxygen for biomedical applications. SPIE Proc. 2007, 6606, 66061E. [CrossRef]

89. Xiao, L.; Gu, L.; Howell, S.B.; Sailor, M. Porous Silicon Nanoparticle Photosensitizers for Singlet Oxygen and Their Phototoxicity against Cancer Cells. ACS Nano 2011, 5, 3651-3659. [CrossRef] [PubMed]

90. Osminkina, L.; Tamarov, K.P.; Sviridov, A.P.; Galkin, R.A.; Gongalsky, M.B.; Solovyev, V.V.; Kudryavtsev, A.A.; Timoshenko, V.Y. Photoluminescent biocompatible silicon nanoparticles for cancer theranostic applications. J. Biophotonics 2012, 5, 529-535. [CrossRef]

91. Fujii, M.; Kovalev, D.; Goller, B.D.; Minobe, S.; Hayashi, S.; Timoshenko, V.Y. Time-resolved photoluminescence studies of the energy transfer from excitons confined in Si nanocrystals to oxygen molecules. Phys. Rev. B 2005, 72, 165321. [CrossRef]

92. Canham, L.T. Nanoscale semiconducting silicon as a nutritional food additive. Nanotechnology 2007, $18,185704$. [CrossRef]

93. Park, J.-H.; Gu, L.; Von Maltzahn, G.; Ruoslahti, E.; Bhatia, S.N.; Sailor, M.J. Biodegradable luminescent porous silicon nanoparticles for in vivo applications. Nat. Mater. 2009, 8, 331-336. [CrossRef] [PubMed]

94. Dolmans, D.E.J.G.J.; Fukumura, D.; Jain, R.K. Photodynamic therapy for cancer. Nat. Rev. Cancer 2003, 3, 380-387. [CrossRef] [PubMed]

95. Gongalsky, M.B.; Kargina, Y.V.; Osminkina, L.; Perepukhov, A.M.; Gulyaev, M.; Vasiliev, A.; Pirogov, Y.A.; Maximychev, A.V.; Timoshenko, V.Y. Porous silicon nanoparticles as biocompatible contrast agents for magnetic resonance imaging. Appl. Phys. Lett. 2015, 107, 233702. [CrossRef]

96. Kargina, Y.V.; Gongalsky, M.B.; Perepukhov, A.M.; Gippius, A.A.; Minnekhanov, A.A.; Zvereva, E.A.; Maximychev, A.V.; Timoshenko, V.Y. Investigation of proton spin relaxation in water with dispersed silicon nanoparticles for potential magnetic resonance imaging applications. J. Appl. Phys. 2018, 123, 1-6. [CrossRef]

97. Von Bardeleben, H.J.; Stiévenard, D.; Grosman, A.; Ortega, C.; Siejka, J. Defects in porous p-type Si: An electron-paramagnetic-resonance study. Phys. Rev. B 1993, 47, 10899-10902. [CrossRef]

98. Pereira, R.N.; Niesar, S.; Wiggers, H.; Brandt, M.S.; Stutzmann, M.S. Depassivation kinetics in crystalline silicon nanoparticles. Phys. Rev. B 2013, 88, 155430. [CrossRef]

99. Rohrer, M.; Bauer, H.; Mintorovitch, J.; Requardt, M.; Weinmann, H.-J. Comparison of Magnetic Properties of MRI Contrast Media Solutions at Different Magnetic Field Strengths. Investig. Radiol. 2005, 40, 715-724. [CrossRef] [PubMed]

100. Araki, K.; Uchiyama, M.K.; Toma, S.; Rodrigues, S.F.; Shimada, A.L.B.; Loiola, R.A.; Rodríguez, H.J.C.; Oliveira, P.V.; Luz, M.S.; Rabbani, S.R.; et al. Ultrasmall cationic superparamagnetic iron oxide nanoparticles as nontoxic and efficient MRI contrast agent and magnetic-targeting tool. Int. J. Nanomed. 2015, 10, 4731-4746. [CrossRef] [PubMed]

101. Kargina, Y.V.; Perepukhov, A.M.; Kharin, A.Y.; Zvereva, E.A.; Koshelev, A.V.; Zinovyev, S.V.; Maximychev, A.V.; Alykova, A.F.; Sharonova, N.V.; Zubov, V.P.; et al. Silicon Nanoparticles Prepared by Plasma-Assisted Ablative Synthesis: Physical Properties and Potential Biomedical Applications. Phys. Status Solidi A 2019, 216, 1800897. [CrossRef]

102. Noginova, N.; Chen, F.; Weaver, T.; Giannelis, E.P.; Bourlinos, A.B.; Atsarkin, V.A. Magnetic resonance in nanoparticles: Between ferro- and paramagnetism. J. Phys. Condens. Matter 2007, 19, 246208. [CrossRef]

103. Kargina, Y.V.; Zinovyev, S.V.; Perepukhov, A.M.; Suslova, E.V.; Ischenko, A.A.; Timoshenko, V.Y. Silicon nanoparticles with iron impurities for multifunctional applications. Funct. Mater. Lett. 2020, 13, 2040007. [CrossRef]

104. Bouchoucha, M.; Van Heeswijk, R.B.; Gossuin, Y.; Kleitz, F.; Fortin, M.-A. Fluorinated Mesoporous Silica Nanoparticles for Binuclear Probes in ${ }^{1} \mathrm{H}$ and ${ }^{19} \mathrm{~F}$ Magnetic Resonance Imaging. Langmuir 2017, 33, 10531-10542. [CrossRef] [PubMed]

105. Greish, K. Enhanced permeability and retention (EPR) effect for anticancer nanomedicine drug targeting. Methods Mol. Biol. 2010, 624, 25-37. [CrossRef]

(C) 2020 by the authors. Licensee MDPI, Basel, Switzerland. This article is an open access article distributed under the terms and conditions of the Creative Commons Attribution (CC BY) license (http://creativecommons.org/licenses/by/4.0/). 